\title{
Rigidity of $\mathrm{SU}(2,2 \mid 2)$-symmetric solutions in Type IIB ${ }^{1}$
}

\section{Eric D'Hoker and Yu Guo}

Department of Physics and Astronomy University of California, Los Angeles, CA 90095, U.S.A.

E-mail: dhoker@physics.ucla.edu, guoyu@physics.ucla.edu

ABSTRACT: We investigate the existence of half-BPS solutions in Type IIB supergravity which are invariant under the superalgebra $\mathrm{SU}(2,2 \mid 2)$ realized on either $A d S_{5} \times S^{2} \times S^{1}$ or $A d S_{5} \times S^{3}$ warped over a Riemann surface $\Sigma$ with boundary. We prove that, in both cases, the only solution is $A d S_{5} \times S^{5}$ itself. We argue that this result provides evidence for the non-existence of fully back-reacted intersecting D3/D7 branes with either $A d S_{5} \times S^{2} \times S^{1} \times \Sigma$ or $A d S_{5} \times S^{3} \times \Sigma$ near-horizon limits.

KEYwords: D-branes, AdS-CFT Correspondence

ARXiv EPRINT: 1001.4808

\footnotetext{
${ }^{1}$ This work was supported in part by National Science Foundation grant PHY-07-57702.
} 


\section{Contents}

1 Introduction 2

1.1 Organization 4

2 Ansatz with $\mathrm{SO}(2,4) \times \mathrm{SO}(3) \times \mathrm{SO}(2)$ symmetry 4

2.1 Type IIB supergravity 4

2.2 The $\mathrm{SO}(2,4) \times \mathrm{SO}(3) \times \mathrm{SO}(2)$-invariant Ansatz 6

$\begin{array}{lll}2.3 & \text { Immediate constraints from the Bianchi identities } & 7\end{array}$

3 The reduced BPS equations $\quad 7$

3.1 Using killing spinors

$\begin{array}{ll}3.2 & \text { The reduced BPS equations }\end{array}$

$\begin{array}{ll}3.3 \text { Symmetries of the reduced BPS equations } & 10\end{array}$

$\begin{array}{lll}3.3 .1 & \text { Continuous symmetries } & 10\end{array}$

$\begin{array}{lll}3.3 .2 & \text { Linear discrete symmetries } & 10\end{array}$

$\begin{array}{lll}\text { 3.3.3 Complex conjugation (or anti-linear) symmetries } & 11\end{array}$

3.4 Further reduction using $\zeta \rightarrow \tau^{(303)} \zeta$ symmetry 11

$\begin{array}{ll}3.5 & \text { Residual symmetries of the reduced form } \\ 3.6 & 12\end{array}$

$\begin{array}{ll}3.6 & \text { Chiral form of the reduced BPS equations } \\ \end{array}$

4 Vanishing G implies the $A d S_{5} \times S^{5}$ solution $\quad 14$

$\begin{array}{lll}4.1 & \text { Using the discrete symmetries of the reduced BPS equations } & 15\end{array}$

$\begin{array}{ll}4.2 \text { Generic solutions when } G=0 & 16\end{array}$

$\begin{array}{ll}4.3 \text { Solution of } A d S_{5} \times S^{5} & 17\end{array}$

5 Metric factors in terms of spinor bilinears $\quad 18$

$\begin{array}{ll}5.1 \text { Example of metric factor calculation } & 18\end{array}$

$\begin{array}{lll}5.2 & \text { Summary of metric factor expressions } & 19\end{array}$

6 Vanishing Hermitian forms $\quad 20$

6.1 Example of Hermitian relation calculation 20

6.2 Summary of all Hermitian relations 21

$\begin{array}{lll}6.3 & \text { Implications for the metric factors } & 22\end{array}$

7 General solution to the reduced BPS equations $\quad 22$

7.1 Solving the Hermitian relations $H_{ \pm}^{(\alpha \beta)}=0 \quad 23$

7.1.1 The generic case for which $\psi_{++} \psi_{+-} \psi_{-+} \psi_{--} \neq 0 \quad 23$

7.1.2 Non-generic cases with vanishing spinor components 24

7.1.3 Two types of solutions 25

7.2 Solving the Hermitian relations $H_{g \pm}^{(\alpha \beta)} \quad 26$

$\begin{array}{lll}7.2 .1 & \text { For the first type of solution } & 26\end{array}$

$\begin{array}{lll}\text { 7.2.2 Vanishing of } g_{9} \text { for first type of solution } & 27\end{array}$ 
7.2.3 Vanishing of $g_{9}$ for second type of solution

$\begin{array}{lll}7.3 & \text { Non-existence of solutions except for } A d S_{5} \times S^{5} & 27\end{array}$

$\begin{array}{lll}\text { 7.3.1 First type of solution } & 27\end{array}$

$\begin{array}{lll}\text { 7.3.2 Second type of solution } & 28\end{array}$

8 Ansatz with $\mathrm{SO}(2,4) \times \mathrm{SO}(4)$ symmetry 28

8.1 Construction of the Ansatz 28

$\begin{array}{ll}8.2 \text { Reducing the BPS equations } & 29\end{array}$

$\begin{array}{lll}\text { 8.2.1 Killing spinors } & 29\end{array}$

$\begin{array}{lll}\text { 8.2.2 The reduced BPS equations } & 30\end{array}$

8.2.3 The chiral form of the reduced BPS equations 30

$\begin{array}{ll}\text { 8.3 Solving the reduced BPS equations } & 31\end{array}$

$\begin{array}{lll}\text { 8.3.1 Using discrete symmetries } & 31\end{array}$

8.3.2 Hermitian forms for metric factors 31

8.3.3 Generic solution to reduced BPS equations 32

8.3.4 Uniqueness of the $A d S_{5} \times S^{5} \quad 32$

A Clifford algebras $\quad \mathbf{3 3}$

A.1 Clifford algebra basis for $A d S_{5} \times S^{2} \times \Sigma \times S^{1}$ space 33

A.2 Clifford algebra basis for $A d S_{5} \times S^{3} \times \Sigma$ space 34

B Reducing the BPS equations: $A d S_{5} \times S^{2} \times \Sigma \times S^{1}$ case $\quad 34$

B.1 The dilatino equation $\quad 35$

$\begin{array}{lll}\text { B.2 The gravitino equation } & 35\end{array}$

$\begin{array}{lll}\text { B.2.1 Assembling the complete gravitino BPS equation } 38 & 38\end{array}$

C Reducing the BPS equations: $A d S_{5} \times S^{3} \times \Sigma$ case $\quad 38$

$\begin{array}{lll}\text { C.1 The dilatino equation } & 38\end{array}$

$\begin{array}{lll}\text { C.2 The gravitino equation } & 39\end{array}$

$\begin{array}{lll}\text { C.2.1 Assembling the complete gravitino BPS equation } & 40\end{array}$

D Calculation of metric factors for $A d S_{5} \times S^{2} \times S^{1} \times \Sigma \quad 40$

D.1 Relations involving $f_{5} \quad 41$

$\begin{array}{lll}\text { D.2 Relations involving } f_{2} & 42\end{array}$

$\begin{array}{lll}\text { D.3 Relations involving } f_{1} & 42\end{array}$

E Vanishing Hermitian forms for $A d S_{5} \times S^{2} \times S^{1} \times \Sigma \quad 43$

E.1 First set of Hermitan relations 43

E.2 Second set of Hermitian relations 44

E.2.1 The $f_{5}$-equation $\quad 45$

E.2.2 The $f_{2}$-equation 45

E.3 Third set of Hermitian relations 46 


\section{Introduction}

The AdS/CFT correspondence [1-3] (for reviews, see [4, 5]) allows one to study gauge theories, in the large $N$ limit and at strong 't Hooft coupling, in terms of dual supergravity solutions in Type IIB or M-theory. Superconformal Yang-Mills theories are of special interest. They are dual to supergravity solutions whose space-time exhibits a (warped) AdS factor, and which are invariant under a conformal super algebra. This case encompasses some of the best understood of all the AdS/CFT dualities.

Substantial progress has been made in recent years in obtaining half-BPS supergravity solutions which are dual to Yang-Mills theories in various dimensions with 16 conformal supersymmetries. Here, the constraints imposed by supersymmetry are strong enough to allow for large classes of exact solutions, while the dynamics remains sufficiently rich to allow for large moduli spaces. A systematic construction, in both Type IIB and M-theory, was initiated in [6], where solutions dual to local half-BPS operator insertions in $\mathcal{N}=4$ super-Yang-Mills were obtained, as were $A d S_{5}$ solutions in M-theory which are dual to $\mathcal{N}=2$ superconformal Yang-Mills theories in 4-dimensions (see also [7]). Subsequently, general classes of exact half-BPS solutions dual to planar interfaces [8-11, 13-15], Wilson loops [16-18], and surface operators [14, 19] were constructed in Type IIB and in Mtheory. These solutions form families, and exhibit a wealth of topological and metrical structure, and are characterized by interesting, and generally complicated, moduli spaces. Generalizations of Janus solutions and their CFT duals to include the $\theta$-angle, as well as a systematic study of their S-duality properties may be found in [20-22].

A general correspondence, in supergravities with 32 supersymmetries, between halfBPS solutions and certain Lie superalgebras with 16 fermionic generators was proposed in [23] for both Type IIB and M-theory. For the Type IIB case of interest here, semi-simple Lie superalgebras $\mathcal{H}$ with 16 fermionic generators which are subalgebras of $P \mathrm{SU}(2,2 \mid 4)$ are related to families of half-BPS solutions to Type IIB supergravity which are invariant under $\mathcal{H}$, and are locally asymptotic to $A d S_{5} \times S^{5}$. Given that there are a finite number of such subalgebras $\mathcal{H}$ (they were completely catalogued in [23], and references therein), a precise classification of such half-BPS solutions becomes available. (Earlier classifications in terms of Killing spinors, their bilinears, and $G$-structures may be found in [24-27].) The special classes of exact solutions discussed in the preceding paragraph all fit into this scheme. The superalgebra classification predicts, however, cases where a suitable subalgebra $\mathcal{H}$ exists, but no corresponding supergravity solutions have been identified yet.

Another approach to the classification of half-BPS solutions is in terms of the nearhorizon limit of various brane intersections, in terms of which many of the known half-BPS solutions may be understood. The brane picture offers a powerful method for understanding the global and qualitative properties of the supergravity solutions, and has led recently to a unified picture of M-theory solutions dual to $\mathcal{N}=2$ superconformal Yang-Mills theories in 4 dimensions [28-30]. All known exact half-BPS solutions have known brane realizations. The brane picture suggests cases, however, of half-BPS near horizon limits of brane intersection where no corresponding supergravity solutions have been found yet.

Perhaps the most important case where the superalgebra and brane pictures appear to be at odds with the absence of any known solutions is for Type IIB supergravity solutions 
which are half-BPS, locally asymptotic to $A d S_{5} \times S^{5}$, and invariant under $\mathrm{SU}(2,2 \mid 2)$. The duals to such solutions would be 4-dimensional $\mathcal{N}=2$ super-Yang-Mills theories with exact conformal invariance. Their brane picture would be in terms of the near-horizon limit of stacks of D3- and D7-branes, intersecting along a 4-dimensional space-time, or equivalently in terms of probe D7-branes inside $A d S_{5}$.

The conflict is as follows. The existence of the subalgebra $\operatorname{SU}(2,2 \mid 2) \subset \operatorname{PSU}(2,2 \mid 4)$ clearly makes it possible to have asymptotically $A d S_{5} \times S^{5}$ half-BPS solutions with this symmetry. The existence and stability of probe D7-branes in $A d S_{5}$ with $\mathrm{SU}(2,2 \mid 2) \times \mathrm{SU}(2)$ symmetry also supports the possibility of having such solutions, though at the expense of including an extra bosonic $\mathrm{SU}(2)$ symmetry group not mandated by superconformal invariance. The question is whether such probe brane solutions extend to fully back-reacted supergravity solutions where the D7-branes dissolve into regular flux solutions.

Several arguments have been presented [19, 31, 32] (following earlier work [33, 34]) that no such fully back-reacted solutions corresponding to D7-branes should exist. A first argument against is that D7-branes produce flavor multiplets [35, 36] transforming under the fundamental representation of the gauge group in the dual gauge theory. Their contribution to the renormalization group $\beta$-function for the gauge coupling is non-vanishing, and thus breaks the exact conformal invariance needed to maintain the $A d S_{5}$ factor on the gravity side. A second argument [37] makes use of local 1/2 BPS-operators: the invariance superalgebra $\mathcal{H}$ for half-BPS solutions which are genuinely asymptotic to $A d S_{5} \times S^{5}$ must have rank strictly less than the rank 6 of $\operatorname{PSU}(2,2 \mid 4)$. This argument leads us to conclude that solutions with $\mathrm{SU}(2,2 \mid 2) \times \mathrm{SU}(2)$ symmetry cannot be asymptotic to $A d S_{5} \times S^{5}$, though there appears to be no obstruction to the existence of locally asymptotic solutions.

In the present paper, we shall focus on half-BPS Type IIB supergravity solutions with bosonic symmetries $\mathrm{SO}(2,4) \times \mathrm{SO}(3) \times \mathrm{SO}(2)$ and $\mathrm{SO}(2,4) \times \mathrm{SO}(4) \times \mathrm{SO}(2)$ respectively corresponding to the maximal bosonic symmetries of $\mathrm{SU}(2,2 \mid 2)$ and $\mathrm{SU}(2,2 \mid 2) \times \mathrm{SU}(2)$. These cases emerge from the general classification of half-BPS solutions via superalgebra arguments [23]. Following the general construction of the space-time Ansätze given there, the symmetries $\mathrm{SO}(2,4) \times \mathrm{SO}(3) \times \mathrm{SO}(2)$ and $\mathrm{SO}(2,4) \times \mathrm{SO}(4) \times \mathrm{SO}(2)$ are realized as isometries of the spaces $A d S_{5} \times S^{2} \times S^{1}$ and $A d S_{5} \times S^{3} \times S^{1}$ respectively.

We shall prove here that the only solution with at least 16 supersymmetries for spacetimes of the form $A d S_{5} \times S^{2} \times S^{1} \times \Sigma$ and $A d S_{5} \times S^{3} \times \Sigma$, invariant respectively under $\mathrm{SO}(2,4) \times \mathrm{SO}(3) \times \mathrm{SO}(2)$ and $\mathrm{SO}(2,4) \times \mathrm{SO}(4)$, is just the maximally supersymmetric solution $A d S_{5} \times S^{5}$. Here, the products $A d S_{5} \times S^{2} \times S^{1}$ and $A d S_{5} \times S^{3}$ are warped over the Riemann surface $\Sigma$. It then follows that no near-horizon limit can exist (in the supergravity limit) for fully back-reacted intersection solutions of D3- and D7-branes with those symmetries and space-time geometries. It is in this sense that the $\mathrm{SU}(2,2 \mid 2)$-invariant solutions are rigid. Note that rigidity of half-BPS solutions has already been encountered in analyzing analogous problems in M-theory [38].

Finally, we note that there is one possible interesting near-horizon limit of D3- and D7-brane fully back-reacted solutions that is not yet excluded by our study. One could imagine a space-time of the form $A d S_{5} \times\left(S^{2} \times_{k} S^{1}\right) \times \Sigma$ where the product $S^{2} \times_{k} S^{1}$ is twisted by the Hopf fibration, with $k \in \mathbf{Z}$ being the monopole number. For $k=0$, 
we recover the case $A d S_{5} \times S^{2} \times S^{1} \times \Sigma$ studied here. For $k \neq 0$ one recovers $S^{3} / Z_{k}$ topologically, but the metric may be deformed to that of a squashed $S^{3}$. For $k=1$, and no squashing, we recover the case $A d S_{5} \times S^{3} \times \Sigma$ studied here. In general, however, the squashing parameter (the ratio of the radius of $S^{1}$ to that of $S^{2}$ ) may be non-trivial, and actually be a function of $\Sigma$. Such cases with non-trivial fibration and squashing require a generalization of the construction methods proposed in [23], and are clearly of physical interest. They are considerably more complicated than the cases treated here; their study will be postponed to future work.

\subsection{Organization}

The remainder of this paper is organized as follows. In section 2, Type IIB supergravity is briefly reviewed, mostly to fix notations, and the $A d S_{5} \times S^{2} \times S^{1} \times \Sigma$ Ansatz is implemented on the Type IIB supergravity fields. In section 3, the BPS equations are reduced on this Ansatz; they are the starting point of the exact solutions. In section 4, we show that the vanishing of the 3 -form field strength implies the maximally supersymmetric solutions $A d S_{5} \times S^{5}$. In section 5, we solve for the metric factors of the $A d S_{5} \times S^{2} \times S^{1}$ spaces, and express them in terms of bilinears in the supersymmetry spinors. In section 6 , the vanishing of several types of Hermitian forms in the supersymmetry spinors are derived from the reduced BPS equations. In section 7, with the help of these vanishing Hermitian forms, it is shown that the only viable solution for the $A d S_{5} \times S^{2} \times S^{1} \times \Sigma$ Ansatz is $A d S_{5} \times S^{5}$. In section 8, the analysis is extended to the $A d S_{5} \times S^{3} \times \Sigma$ Ansatz. It is shown that also here the only viable half-BPS solution is $A d S_{5} \times S^{5}$. In appendix A, we give Clifford algebra representations adapted to the two different Ansätze. In appendices B and C, we provide the details of reducing BPS equations for the two different Ansätze. In appendix D and E, technical details of solving metric factors and vanishing Hermitian forms are presented.

\section{Ansatz with $\mathrm{SO}(2,4) \times \mathrm{SO}(3) \times \mathrm{SO}(2)$ symmetry}

In this section, we briefly review Type IIB supergravity, and construct the Ansatz for supergravity solutions with $\mathrm{SO}(2,4) \times \mathrm{SO}(3) \times \mathrm{SO}(2)$ symmetry.

\section{$2.1 \quad$ Type IIB supergravity}

The canonical fields of Type IIB supergravity are the metric $g_{M N}$, and the 4 -form $C_{(4)}$, both of which are real fields, the axion/dilaton scalar field $B$, and the 2 -form $B_{(2)}$, both of which are complex fields, as well as the dilatino $\lambda$ and gravitino $\psi_{M}$ spinor fields. Our conventions are those of $[39,40]$ (see also [41]). It is convenient to formulate Type IIB supergravity in terms of the field strengths, Bianchi identities, and field equations. For the axion/dilaton, we introduce the 1 -form fields $P, Q$,

$$
\begin{aligned}
& P=f_{B}^{2} d B \quad f_{B}^{2}=\left(1-|B|^{2}\right)^{-1} \\
& Q=f_{B}^{2} \operatorname{Im}(B d \bar{B})
\end{aligned}
$$


while for the 2- and 4-form fields we introduce the field strengths,

$$
\begin{aligned}
G & =f_{B}\left(F_{(3)}-B \bar{F}_{(3)}\right) & F_{(3)}=d B_{(2)} \\
F_{(5)} & =d C_{(4)}+\frac{i}{16}\left(B_{(2)} \wedge \bar{F}_{(3)}-\bar{B}_{(2)} \wedge F_{(3)}\right) &
\end{aligned}
$$

The scalar field $B$ is related to the complex string coupling $\tau$, the axion $\chi$, and dilaton $\Phi$. In terms of the composite fields $P, Q$, and $G$, there are Bianchi identities given as follows,

$$
\begin{aligned}
0 & =d P-2 i Q \wedge P \\
0 & =d Q+i P \wedge \bar{P} \\
0 & =d G-i Q \wedge G+P \wedge \bar{G} \\
0 & =d F_{(5)}-\frac{i}{8} G \wedge \bar{G}
\end{aligned}
$$

The field strength $F_{(5)}$ is required to be self-dual,

$$
F_{(5)}=* F_{(5)}
$$

The field equations are given by,

$$
\begin{aligned}
0= & \nabla^{M} P_{M}-2 i Q^{M} P_{M}+\frac{1}{24} G_{M N P} G^{M N P} \\
0= & \nabla^{P} G_{M N P}-i Q^{P} G_{M N P}-P^{P} \bar{G}_{M N P}+\frac{2}{3} i F_{(5) M N P Q R} G^{P Q R} \\
0= & R_{M N}-P_{M} \bar{P}_{N}-\bar{P}_{M} P_{N}-\frac{1}{6}\left(F_{(5)}^{2}\right)_{M N} \\
& -\frac{1}{8}\left(G_{M}{ }^{P Q} \bar{G}_{N P Q}+\bar{G}_{M}{ }^{P Q} G_{N P Q}\right)+\frac{1}{48} g_{M N} G^{P Q R} \bar{G}_{P Q R}
\end{aligned}
$$

The BPS equations for bosonic solutions are obtained by setting the supersymmetry variations $\delta \lambda$ and $\delta \psi_{M}$ of the fermionic fields to zero, in the presence of vanishing fermi fields, ${ }^{1}$

$$
\begin{aligned}
& 0=i(\Gamma \cdot P) \mathcal{B}^{-1} \varepsilon^{*}-\frac{i}{24}(\Gamma \cdot G) \varepsilon \\
& 0=D_{M} \varepsilon+\frac{i}{480}\left(\Gamma \cdot F_{(5)}\right) \Gamma_{M} \varepsilon-\frac{1}{96}\left(\Gamma_{M}(\Gamma \cdot G)+2(\Gamma \cdot G) \Gamma^{M}\right) \mathcal{B}^{-1} \varepsilon^{*}
\end{aligned}
$$

Here, $\mathcal{B}$ is the complex conjugation matrix of the Clifford algebra. ${ }^{2}$

Type IIB supergravity is invariant under $\mathrm{SU}(1,1) \sim \mathrm{SL}(2, \mathbf{R})$ symmetry, which leaves $g_{M N}$ and $C_{(4)}$ invariant, acts by Möbius transformation on the field $B$, and linearly on $B_{(2)}$,

$$
\begin{aligned}
B & \rightarrow \frac{u B+v}{\bar{v} B+\bar{u}} \\
B_{(2)} & \rightarrow u B_{(2)}+v \bar{B}_{(2)}
\end{aligned}
$$

\footnotetext{
${ }^{1}$ Throughout, we shall use the notation $\Gamma \cdot T \equiv \Gamma^{M_{1} \cdots M_{p}} T_{M_{1} \cdots M_{p}}$ for the contraction of any antisymmetric tensor field $T$ of rank $p$ and the $\Gamma$-matrix of the same rank.

${ }^{2}$ The matrix $\mathcal{B}$ is defined by $\mathcal{B} \mathcal{B}^{*}=I$ and $\mathcal{B} \Gamma^{M} \mathcal{B}^{-1}=\left(\Gamma^{M}\right)^{*}$; see appendix A for our $\Gamma$-matrix conventions. Throughout, complex conjugation will be denoted by bar for functions, and by star for spinors.
} 
with $u, v \in \mathbf{C}$ and $\bar{u} u-\bar{v} v=1$. The transformation rules for the composite fields are [39],

$$
\begin{aligned}
P & \rightarrow e^{2 i \theta} P \\
Q & \rightarrow Q+d \theta \\
G & \rightarrow e^{i \theta} G
\end{aligned}
$$

where the phase $\theta$ is defined by

$$
e^{2 i \theta}=\frac{v \bar{B}+u}{\bar{v} B+\bar{u}}
$$

In this form, the transformation rules clearly exhibit the $\mathrm{U}(1)_{q}$ gauge transformation that accompanies the global $\mathrm{SU}(1,1)$ transformations.

\subsection{The $\mathrm{SO}(2,4) \times \mathrm{SO}(3) \times \mathrm{SO}(2)$-invariant Ansatz}

We seek the most general Ansatz in Type IIB supergravity with $\mathrm{SO}(2,4) \times \mathrm{SO}(3) \times \mathrm{SO}(2)$ symmetry. The $\mathrm{SO}(2,4)$-factor requires the geometry to contain an $A d S_{5}$-factor, while the $\mathrm{SO}(3)$ - and $\mathrm{SO}(2)$-factors respectively require $S^{2}$ - and $S^{1}$-factors, all warped over a 2-dimensional surface $\Sigma$. The total space-time then has the structure,

$$
A d S_{5} \times S^{2} \times \Sigma \times S^{1}
$$

Following the general constructing as in [10], the Ansatz will be given in terms of $\mathrm{SO}(2,4) \times$ $\mathrm{SO}(3) \times \mathrm{SO}(2)$ invariant supergravity fields, which are functions on the surface $\Sigma$. The Ansatz for the metric is given by,

$$
d s^{2}=f_{5}^{2} d s_{A d S_{5}}^{2}+f_{2}^{2} d s_{S^{2}}^{2}+d s_{\Sigma}^{2}+f_{1}^{2} d s_{S^{1}}^{2}
$$

where $f_{5}, f_{2}$ and $f_{1}$ are real functions on $\Sigma$. Orthonormal frames are defined by,

$$
\begin{array}{rlrl}
e^{m} & =f_{5} \hat{e}^{m} & m & =0,1,2,3,4 \\
e^{i} & =f_{2} \hat{e}^{i} & i & =5,6 \\
e^{a} & =\rho \hat{e}^{a} & a & =7,8 \\
e^{9} & =f_{1} \hat{e}^{9} &
\end{array}
$$

Here, the metrics $d s_{A d S_{5}}^{2}, d s_{S^{2}}^{2}$, and $d s_{S^{1}}^{2}$, as well as the orthonormal frames $\hat{e}^{m}, \hat{e}^{i}$, and $\hat{e}^{9}$ refer to the spaces $A d S_{5}, S^{2}$, and $S^{1}$ with unit radius. In particular, we have

$$
\begin{aligned}
d s_{A d S_{5}}^{2} & =\eta_{m n} \hat{e}^{m} \otimes \hat{e}^{n} \\
d s_{S^{2}}^{2} & =\delta_{i j} \hat{e}^{i} \otimes \hat{e}^{j} \\
d s_{\Sigma}^{2} & =\delta_{a b} e^{a} \otimes e^{b} \\
d s_{S^{1}}^{2} & =\hat{e}^{9} \otimes \hat{e}^{9}
\end{aligned}
$$

where $\eta=\operatorname{diag}[-+++]$. Similarly, the dilaton/axion field $B$ is a function of $\Sigma$ only. As a result, the 1-forms $P$ and $Q$ have no component along $e^{9}$, so that $p_{9}=q_{9}=0$ and their structures is simply given by,

$$
\begin{aligned}
& P=p_{a} e^{a} \\
& Q=q_{a} e^{a}
\end{aligned}
$$


Finally, the most general Ansätze for the 3 - and 5-form fields $G$ and $F_{(5)}$ consistent with $\mathrm{SO}(2,4) \times \mathrm{SO}(3) \times \mathrm{SO}(2)$ invariance, are given by, ${ }^{3}$

$$
\begin{aligned}
G & =i g_{\tilde{a}} e^{56 \tilde{a}}+h e^{789}=i g_{a} e^{56 a}+i g_{9} e^{569}+h e^{789} \\
F_{(5)} & =f\left(e^{01234}-e^{56789}\right)
\end{aligned}
$$

Note that the components of $G$ involving any of the directions $0,1,2,3,4$ must vanish by $\mathrm{SO}(2,4)$ symmetry, and that the remaining terms are the only allowed ones by $\mathrm{SO}(3)$ invariance. $\mathrm{By} \mathrm{SO}(2,4) \times \mathrm{SO}(3) \times \mathrm{SO}(2)$-invariance, the coefficient functions $p_{a}, q_{a}, g_{a}, g_{9}, h$, and $f$ depend only on $\Sigma$. The functions $f, q_{a}$ are real, while $p_{a}, h, g_{a}, g_{9}$ are complex-valued.

\subsection{Immediate constraints from the Bianchi identities}

There are two immediate consequences of the Bianchi identities and field definitions for the coefficient functions of the Ansatz.

- The coefficient $g_{9}$ is determined by the field definitions. This may be shown directly by observing that the $g_{9}$-term in $G$ can arise only from a contribution $b_{0} \hat{e}^{569}$ to $F_{(3)}$ where $b_{0}$ is a complex function of $\Sigma$. Closure of $F_{(3)}$ requires $b_{0}$ to be constant, so that $g_{9}$ is given by,

$$
g_{9}=-i \frac{f_{B}}{f_{2}^{2} f_{1}}\left(b_{0}-B b_{0}^{*}\right)
$$

with $b_{0}$ an arbitrary complex constant. Note that the corresponding $B_{(2)}$-field is given by $b_{0} x^{9} \hat{e}^{56}$. This form is invariant up to a gauge transformation under the action of $\mathrm{SO}(2)$, namely under a constant shift in the coordinate $x^{9}$ of the circle $S^{1}$.

- The Bianchi identity for $F_{(5)}$ reduces to $d F_{(5)}=0$, since $G \wedge \bar{G}$ vanishes identically on the Ansatz. Using the closure of $\hat{e}^{01234}$, we find that $f f_{5}^{5}$ must be constant,

$$
f f_{5}^{5}=c_{0}
$$

where $c_{0}$ is an arbitrary real constant.

\section{The reduced BPS equations}

Half-BPS configurations and solutions in Type IIB supergravity with vanishing Fermi fields are such that the BPS equations (2.11) for the spinor $\varepsilon$ admit 16 linearly independent solutions. The spinor $\varepsilon$ is covariant under $\mathrm{SO}(2,4) \times \mathrm{SO}(3) \times \mathrm{SO}(2)$, and may be built from the Killing spinors on the space $A d S_{5} \times S^{2} \times S^{1}$, where each factor space has unit radius. Once this structure is in place, the reduction of the full BPS equations of (2.11) is standard.

\footnotetext{
${ }^{3}$ Throughout the $\mathrm{SO}(2,4) \times \mathrm{SO}(3) \times \mathrm{SO}(2)$ symmetry case, indices $a, b$ will run over the values 7,8 , while indices $\tilde{a}, \tilde{b}$ will run over the values $7,8,9$.
} 


\subsection{Using killing spinors}

We begin by constructing suitable Killing spinors on $A d S_{5} \times S^{2}$. We define an 8-dimensional spinor representation $\chi^{\eta_{1}, \eta_{2}}$ of $\mathrm{SO}(2,4) \times \mathrm{SO}(3)$ by the following equations $[10,13]$,

$$
\begin{array}{rlrl}
\left(\hat{\nabla}_{m}-\frac{1}{2} \eta_{1} \gamma_{m} \otimes I_{2}\right) \chi^{\eta_{1}, \eta_{2}} & =0 & & m=0,1,2,3,4 \\
\left(\hat{\nabla}_{i}-\frac{i}{2} \eta_{2} I_{4} \otimes \gamma_{i}\right) \chi^{\eta_{1}, \eta_{2}} & =0 & i & =5,6
\end{array}
$$

Here, $\gamma_{m}$ and $\gamma_{i}$ are the Dirac matrices on $A d S_{5}$ and $S^{2}$ respectively (see appendix A.1). Integrability requires $\eta_{1}^{2}=\eta_{2}^{2}=1$, and the respective solution spaces are indeed of dimension 4 and 2 , so that $\chi^{\eta_{1}, \eta_{2}}$ has 8 independent solutions.

The chirality matrix $\gamma_{(2)}$ of the $\gamma_{i}$-Dirac algebra produces a reversal of the sign of $\eta_{2}$, allowing us to make the following identification,

$$
\gamma_{(2)} \chi^{\eta_{1}, \eta_{2}}=\chi^{\eta_{1},-\eta_{2}}
$$

Charge conjugation, under which $\chi \rightarrow \chi^{c}$, with

$$
\left(\chi^{c}\right)^{\eta_{1}, \eta_{2}}=\left(B_{(1)} \otimes B_{(2)}\right)^{-1}\left(\chi^{\eta_{1}, \eta_{2}}\right)^{*}
$$

reverses the signs of both $\eta_{1}$ and $\eta_{2}$, so that $\left(\chi^{c}\right)^{\eta_{1}, \eta_{2}}$ is proportional to $\chi^{-\eta_{1},-\eta_{2}}$. The proportionality factor depends on $\eta_{1}, \eta_{2}$, and may be determined once a sign convention has been chosen for one of the four components of $\chi^{\eta_{1}, \eta_{2}}$. It will be convenient to make the choice $\chi^{c++}=\chi^{--}$. The remaining components are then related by using the $\gamma_{(2)}$-chirality matrix, and the properties of the charge conjugation matrices $B_{(1)}$ and $B_{(2)}$. For example, we have $\chi^{c+-}=\left(B_{(1)} \otimes B_{(2)}\right)^{-1} \gamma_{(2)} \chi^{*++}$ by the definition of $\chi^{c}$, and the action of $\gamma_{(2)}$. Commuting the $\gamma$-matrix past the $B$-matrices, we have $\chi^{c+-}=-\gamma_{(2)}\left(B_{(1)} \otimes B_{(2)}\right)^{-1} \chi^{*++}$, which in turn equals $-\chi^{-+}$. The remaining relations may be similarly obtained, and we find,

$$
\left(\chi^{c}\right)^{\eta_{1}, \eta_{2}}=\eta_{2} \chi^{-\eta_{1},-\eta_{2}}
$$

a relation which is now valid for all four assignments of $\eta_{1}, \eta_{2}$.

The Killing spinors on $S^{1}$ are solutions to the equation,

$$
\left(\hat{\nabla}_{9}-\frac{i}{2} \eta_{3}\right) \chi^{\eta_{3}}=0
$$

Here, we have $\eta_{3}= \pm$, and $\chi^{\eta_{3}}$ consists of just a single function, since the irreducible spinor representation of $\mathrm{SO}(2)$ on $S^{1}$ are 1-dimensional. By choosing a proper overall phase convention, we may set $\left(\chi^{\eta_{3}}\right)^{*}=\chi^{-\eta_{3}}$. Note that the values of $\eta_{3}$ are not required by integrability, since the above equation is integrable for all values of $\eta_{3}$. Rather, the values $\eta_{3}= \pm 1$ naturally correspond to a double-valued representation, suitable for spinors. Other odd values of $\eta_{3}$ are allowed as well, but will be equivalent to our choice, upon redefinition of the metric function $f_{1}$ of the circle $S^{1}$. 
Putting all together, the full 32-component spinor $\varepsilon$ may thus be decomposed as follows in terms of Killing spinors on $A d S_{5} \times S^{2} \times S^{1}$,

$$
\varepsilon=\sum_{\eta_{1}, \eta_{2}, \eta_{3}} \chi^{\eta_{1}, \eta_{2}} \chi^{\eta_{3}} \otimes\left(\zeta_{\eta_{1}, \eta_{2}, \eta_{3}} \otimes u_{+}+\zeta_{\eta_{1}, \eta_{2}, \eta_{3}}^{\prime} \otimes u_{-}\right)
$$

where we have used the following basis,

$$
u_{+} \equiv\left(\begin{array}{l}
1 \\
0
\end{array}\right) \quad u_{-} \equiv\left(\begin{array}{l}
0 \\
1
\end{array}\right)
$$

Here, $\zeta_{\eta_{1}, \eta_{2}, \eta_{3}}$, and $\zeta_{\eta_{1}, \eta_{2}, \eta_{3}}^{\prime}$ are independent 2-dimensional spinor functions of $\Sigma$. The counting of components is as follows: $\chi^{\eta_{1} \eta_{2}}$ has 8 components, $\chi^{\eta_{3}}$ has just one component, and the factor in parentheses in (3.6) a general 4-component spinor. The 10-dimensional chirality condition $\Gamma^{11} \varepsilon=-\varepsilon$ relates $\zeta^{\prime}$ to $\zeta$ since we have $\Gamma^{11}=I_{4} \otimes I_{2} \otimes I_{2} \otimes \sigma_{2}$, and thus,

$$
\Gamma^{11} \varepsilon=\sum_{\eta_{1}, \eta_{2}, \eta_{3}} \chi^{\eta_{1}, \eta_{2}} \chi^{\eta_{3}} \otimes\left(i \zeta_{\eta_{1}, \eta_{2}, \eta_{3}} \otimes u_{-}-i \zeta_{\eta_{1}, \eta_{2}, \eta_{3}}^{\prime} \otimes u_{+}\right)
$$

As a result, we have $\zeta_{\eta_{1}, \eta_{2}, \eta_{3}}^{\prime}=-i \zeta_{\eta_{1}, \eta_{2}, \eta_{3}}$. By absorbing a constant phase into the $\zeta$ spinor, the last entry of the tensor product in the 10-dimensional spinor may now be recast in terms of a constant spinor $\theta$, defined by

$$
\theta=e^{i \pi / 4} u_{+}+e^{-i \pi / 4} u_{-}
$$

Its overall phase was chosen so that $\theta$ obeys $\sigma^{1} \theta=\theta^{*}, \sigma^{2} \theta=-\theta$, and $\sigma^{3} \theta=i \theta^{*}$. The full 10-dimensional spinor then takes the final form we shall use,

$$
\varepsilon=\sum_{\eta_{1}, \eta_{2}, \eta_{3}} \chi^{\eta_{1}, \eta_{2}} \chi^{\eta_{3}} \otimes \zeta_{\eta_{1}, \eta_{2}, \eta_{3}} \otimes \theta
$$

It will be convenient to use matrix notation for the action of the indices $\eta_{1}, \eta_{2}, \eta_{3}$,

$$
\tau^{(i j k)} \equiv \tau^{i} \otimes \tau^{j} \otimes \tau^{k} \quad i, j, k=0,1,2,3
$$

where $\tau^{0}=I_{2}$, and $\tau^{i}$ with $i=1,2,3$ are the Pauli matrices in the standard basis. Multiplication by $\tau^{(i j k)}$ is defined as follows,

$$
\left(\tau^{(i j k)} \zeta\right)_{\eta_{1}, \eta_{2}, \eta_{3}} \equiv \sum_{\eta_{1}^{\prime}, \eta_{2}^{\prime}, \eta_{3}^{\prime}}\left(\tau^{i}\right)_{\eta_{1} \eta_{1^{\prime}}}\left(\tau^{j}\right)_{\eta_{2} \eta_{2^{\prime}}}\left(\tau^{k}\right)_{\eta_{3} \eta_{3^{\prime}}} \zeta_{\eta_{1}^{\prime}, \eta_{2}^{\prime}, \eta_{3}^{\prime}}
$$

Henceforth, we shall use matrix notation for $\tau$ and suppress the indices $\eta$.

\subsection{The reduced BPS equations}

Details of the reduction of the BPS-equations of (2.11) have been deferred to appendix B. The final results are as follows. The dilatino BPS equation is given by,

$$
\text { (d) } \quad 0=4 p_{a} \sigma^{a} \sigma^{2} \zeta^{*}+g_{\tilde{a}} \tau^{(131)} \sigma^{\tilde{a}} \zeta+h \tau^{(121)} \zeta
$$


while the gravitino equation decomposes into a system of 4 equations,

$$
\begin{aligned}
(m) \quad 0= & \frac{i}{2 f_{5}} \tau^{(300)} \zeta+\frac{D_{a} f_{5}}{2 f_{5}} \tau^{(010)} \sigma^{a} \zeta-\frac{i}{2} f \zeta \\
& +\frac{1}{16}\left(i g_{\tilde{a}} \tau^{(121)} \sigma^{\tilde{a}} \sigma^{2} \zeta^{*}+i h \tau^{(131)} \sigma^{2} \zeta^{*}\right) \\
(i) \quad 0= & \frac{i}{2 f_{2}} \tau^{(030)} \zeta+\frac{D_{a} f_{2}}{2 f_{2}} \tau^{(010)} \sigma^{a} \zeta+\frac{i}{2} f \zeta \\
& +\frac{1}{16}\left(-3 i g_{\tilde{a}} \tau^{(121)} \sigma^{\tilde{a}} \sigma^{2} \zeta^{*}+i h \tau^{(131)} \sigma^{2} \zeta^{*}\right) \\
(9) \quad 0= & \frac{i}{2 f_{1}} \tau^{(013)} \sigma^{3} \zeta+\frac{D_{a} f_{1}}{2 f_{1}} \tau^{(010)} \sigma^{a} \zeta+\frac{i}{2} f \zeta \\
& +\frac{1}{16}\left(-3 i g_{9} \tau^{(121)} \sigma^{3} \sigma^{2} \zeta^{*}+i g_{a} \tau^{(121)} \sigma^{a} \sigma^{2} \zeta^{*}-3 i h \tau^{(131)} \sigma^{2} \zeta^{*}\right) \\
(a) \quad 0 & \left(D_{a}+\frac{i}{2} \hat{\omega}_{a} \sigma^{3}\right) \zeta-\frac{i q_{a}}{2} \zeta+\frac{i f}{2} \tau^{(010)} \sigma_{a} \zeta \\
& +\frac{1}{16}\left(3 g_{a} \tau^{(131)} \sigma^{2} \zeta^{*}-g_{\tilde{b}} \tau^{(131)} \sigma_{a}^{\tilde{b}} \sigma^{2} \zeta^{*}-3 h \tau^{(121)} \sigma_{a} \sigma^{2} \zeta^{*}\right)
\end{aligned}
$$

\subsection{Symmetries of the reduced BPS equations}

The symmetries of the reduced BPS equations will play a fundamental role in the analysis of half-BPS solutions. It will be natural to separate the symmetries into three groups: continuous symmetries; linear discrete symmetries which map $\zeta$ to a linear function of $\zeta$; and complex conjugations which map $\zeta$ to a linear function of $\zeta^{*}$.

\subsubsection{Continuous symmetries}

One manifest continuous symmetry consists of the U(1) frame rotations on the orthogonal frame $e^{a}$ on $\Sigma$.

The axion/dilaton field $B$ transforms non-linearly under the continuous $S$-duality group $\mathrm{SU}(1,1)$ of Type IIB supergravity, and the field $B$ takes values in the coset $\mathrm{SU}(1,1) / \mathrm{U}(1)_{q}$. Thus, $\mathrm{SU}(1,1)$ transformations on the $B$-field and on other fields are accompanied by local $\mathrm{U}(1)_{q}$ gauge transformations, given in (2.13) and (2.14). This $\mathrm{U}(1)_{q}$ survives the reduction to our Ansatz, and induces the following transformations,

$$
\begin{array}{rlrl}
\zeta & \rightarrow e^{i \theta / 2} \zeta & g_{a} & \rightarrow e^{i \theta} g_{a} \\
q_{a} & \rightarrow q_{a}+D_{a} \theta & g_{9} & \rightarrow e^{i \theta} g_{9} \\
p_{a} & \rightarrow e^{2 i \theta} p_{a} & h & \rightarrow e^{i \theta} h
\end{array}
$$

The real function $\theta$ depends on the $\mathrm{SU}(1,1)$ transformation, as well as on the field $B$.

\subsubsection{Linear discrete symmetries}

The following linear transformations on $\zeta$ are symmetries of all the BPS equations, upon leaving the metric factors $f_{1}, f_{2}, f_{5}$, the flux fields $f, g_{a}, g_{9}, h, p_{a}, q_{a}$ unchanged,

$$
\zeta \rightarrow \zeta^{\prime}=S \zeta \quad S \in \mathcal{S}_{0} \equiv\left\{I, \tau^{(303)}, i \tau^{(300)}, i \tau^{(003)}\right\}
$$


The set $\mathcal{S}_{0}$ forms an Abelian group. The BPS equations have further linear symmetries $\zeta \rightarrow \zeta^{\prime}=S \zeta$, given by,

$$
S \in \mathcal{S}_{1} \equiv\left\{\tau^{(331)} \sigma^{3}, \tau^{(332)} \sigma^{3}, i \tau^{(031)} \sigma^{3}, i \tau^{(032)} \sigma^{3}\right\}
$$

The transformations in $\mathcal{S}_{1}$ leave $f_{1}, f_{2}, f_{5}$, and $f, g_{a}, h, p_{a}, q_{a}$ unchanged but, in contrast with the transformations in $\mathcal{S}_{0}$, must be accompanied by a sign reversal $g_{9} \rightarrow g_{9}^{\prime}=-g_{9}$. Thus, the generators of $\mathcal{S}_{1}$ are genuine symmetries of the bosonic supergravity fields only if $g_{9}=0$. The union $\mathcal{S}_{0} \cup \mathcal{S}_{1}$ forms a non-Abelian group, whose generators may be chosen to be

$$
\begin{aligned}
& T_{1} \zeta=\tau^{(303)} \zeta \\
& T_{2} \zeta=i \tau^{(300)} \zeta \\
& T_{3} \zeta=\tau^{(331)} \sigma^{3} \zeta
\end{aligned}
$$

\subsubsection{Complex conjugation (or anti-linear) symmetries}

The BPS equations are invariant under 8 discrete complex conjugations, which may be viewed as the compositions of the 8 transformations of $\mathcal{S}_{0} \cup \mathcal{S}_{1}$ with a single complex conjugation. It will be useful to record a more general transformation, in which we compose the discrete complex conjugation with an arbitrary continuous $\mathrm{U}(1)_{q}$ transformation, which gives,

$$
\begin{aligned}
\zeta \rightarrow T_{4} \zeta=\zeta^{\prime}=e^{i \theta} \tau^{(030)} \sigma^{1} \zeta^{*} \quad & g_{a} \rightarrow g_{a}^{\prime}=e^{2 i \theta} g_{a}^{*} \\
g_{9} & \rightarrow g_{9}^{\prime}=-e^{2 i \theta} g_{9}^{*} \\
& h \rightarrow h^{\prime}=e^{2 i \theta} h^{*} \\
p_{a} & \rightarrow p_{a}^{\prime}=e^{4 i \theta} p_{a}^{*} \\
& q_{a} \rightarrow q_{a}^{\prime}=-q_{a}+2 D_{a} \theta
\end{aligned}
$$

The pure complex conjugation corresponds to the special case where $\theta=0$.

\subsection{Further reduction using $\zeta \rightarrow \tau^{(303)} \zeta$ symmetry}

The transformations in $\mathcal{S}_{1}$ are genuine symmetries of the BPS equations only if $g_{9}=0$, while the complex conjugations are symmetries only if the dilaton/axion and flux fields obey reality conditions $p_{a}^{*}=p_{a}, q_{a}=0, g_{a}^{*}=g_{a}, g_{9}^{*}=-g_{9}$, and $h^{*}=h$, up to $\mathrm{U}(1)_{q}$. For general bosonic fields, only the transformations in $\mathcal{S}_{0}$ are genuine symmetries of the BPS equations. Only the transformation $\zeta \rightarrow \tau^{(303)} \zeta$ commutes with the BPS differential operator acting on real linear combinations, so that this is the only symmetry that may be diagonalized simultaneously with the BPS operator. (Note that the other two non-trivial symmetries of $\mathcal{S}_{0}$ square to $-I$, and admit no real eigenvalues.) Thus, we may now analyze separately the restrictions of the BPS equations to the two eigenspaces of $\tau^{(303)}$, given $\nu= \pm 1$

$$
\tau^{(303)} \zeta=\nu \zeta
$$


Redefining the non-vanishing component of the spinor $\zeta$ in terms of a spinor with only two indices (which, by abuse of notation, we denote again by $\zeta$ ), we have,

$$
\nu=1\left\{\begin{array}{l}
\zeta_{++} \equiv \zeta_{+++} \\
\zeta_{+-} \equiv \zeta_{+-+} \\
\zeta_{-+} \equiv \zeta_{-+-} \\
\zeta_{--} \equiv \zeta_{---}
\end{array} \quad \nu=-1\left\{\begin{array}{l}
\zeta_{++} \equiv \zeta_{++-} \\
\zeta_{+-} \equiv \zeta_{+--} \\
\zeta_{-+} \equiv \zeta_{-++} \\
\zeta_{--} \equiv \zeta_{--+}
\end{array}\right.\right.
$$

In terms of this two-index spinor $\zeta$, the BPS equations become,

$$
0=4 p_{a} \sigma^{a} \sigma^{2} \zeta^{*}+g_{\tilde{a}} \tau^{(13)} \sigma^{\tilde{a}} \zeta+h \tau^{(12)} \zeta
$$

$(m)$

$$
\begin{aligned}
0= & \frac{i}{2 f_{5}} \tau^{(30)} \zeta+\frac{D_{a} f_{5}}{2 f_{5}} \tau^{(01)} \sigma^{a} \zeta-\frac{i}{2} f \zeta \\
& +\frac{1}{16}\left(i g_{\tilde{a}} \tau^{(12)} \sigma^{\tilde{a}} \sigma^{2} \zeta^{*}+i h \tau^{(13)} \sigma^{2} \zeta^{*}\right)
\end{aligned}
$$

$$
\begin{aligned}
0= & \frac{i}{2 f_{2}} \tau^{(03)} \zeta+\frac{D_{a} f_{2}}{2 f_{2}} \tau^{(01)} \sigma^{a} \zeta+\frac{i}{2} f \zeta \\
& +\frac{1}{16}\left(-3 i g_{\tilde{a}} \tau^{(12)} \sigma^{\tilde{a}} \sigma^{2} \zeta^{*}+i h \tau^{(13)} \sigma^{2} \zeta^{*}\right)
\end{aligned}
$$

$$
\begin{aligned}
0= & \frac{i \nu}{2 f_{1}} \tau^{(31)} \sigma^{3} \zeta+\frac{D_{a} f_{1}}{2 f_{1}} \tau^{(01)} \sigma^{a} \zeta+\frac{i}{2} f \zeta \\
& +\frac{1}{16}\left(i g_{a} \tau^{(12)} \sigma^{a} \sigma^{2} \zeta^{*}-3 i g_{9} \tau^{(12)} \sigma^{3} \sigma^{2} \zeta^{*}-3 i h \tau^{(13)} \sigma^{2} \zeta^{*}\right)
\end{aligned}
$$

(a) $\quad 0=\left(D_{a}+\frac{i}{2} \hat{\omega}_{a} \sigma^{3}\right) \zeta-\frac{i q_{a}}{2} \zeta+\frac{i f}{2} \tau^{(01)} \sigma_{a} \zeta$

$$
+\frac{1}{16}\left(3 g_{a} \tau^{(13)} \sigma^{2} \zeta^{*}-g_{\tilde{b}} \tau^{(13)} \sigma_{a}^{\tilde{b}} \sigma^{2} \zeta^{*}-3 h \tau^{(12)} \sigma_{a} \sigma^{2} \zeta^{*}\right)
$$

The matrices $\tau^{(i j)}$ act on the two-index spinor $\zeta$ in the obvious manner.

Remarkably, the dependence on $\nu$ of the reduced BPS equations is entirely concentrated in the first term of equation (9) in (3.21), and may be undone by changing the sign of the metric factor $f_{1}$. As only the square $f_{1}^{2}$ enters into any of the supergravity fields, this sign redefinition is immaterial, and the projections $\nu= \pm 1$ represent the same bosonic supergravity fields. A solution to the reduced BPS equations for $\nu=+1$ thus automatically produces another solution with the same bosonic fields but with $\nu=-1$. In this manner, a systematic doubling of the number of spinor solutions is produced.

As a corollary, this doubling up of the number of spinor solutions implies that any solution to the BPS equations with $\nu=+1$ effectively produces 16 linearly independent solutions of the full BPS equations, and thus generates a half-BPS solution.

\subsection{Residual symmetries of the reduced form}

Having projected $\zeta$ onto the two eigenspaces $\tau^{(303)} \zeta=\nu \zeta$, any residual symmetry of (3.21) must map these eigenspace into themselves, requiring the transformation to commute with $\tau^{(303)}$. The transformations $\mathrm{U}(1)_{q}$ thus continue to be symmetries of (3.21). The remaining 
elements in $\mathcal{S}_{0}$, namely $i \tau^{(300)}$ and $i \tau^{(003)}$ map between identical $\nu$, and reduce to the following transformations on two-index $\zeta$ variables,

$$
\begin{aligned}
& i \tau^{(300)} \rightarrow i \tau^{(30)} \\
& i \tau^{(003)} \rightarrow i \nu \tau^{(30)}
\end{aligned}
$$

both of which are symmetries of the further reduced BPS equations (3.21). The transformations in $\mathcal{S}_{1}$ all anti-commute with $\tau^{(303)}$, thus map the system with $\nu$ to the system with $-\nu$, and do not yield symmetries of the further reduced BPS equations (3.21).

Amongst the complex conjugation symmetries of the original reduced BPS equations (3.13) and (3.14), only the transformation of (3.19), and its images under $\mathcal{S}_{0}$, commute with $\tau^{(303)}$. The transformation of (3.19) acting on 2-index $\zeta$ variables is given by,

$$
\zeta \rightarrow \zeta^{\prime}=e^{i \theta} \tau^{(03)} \sigma^{1} \zeta^{*}
$$

and is a residual symmetry of the further reduced BPS equations (3.21).

\subsection{Chiral form of the reduced BPS equations}

It will be convenient to separate the two components of the 2-dimensional spinors $\zeta_{\eta_{1}, \eta_{2}, \eta_{3}}$, or of their two-index reductions $\zeta_{\eta_{1}, \eta_{2}}$. This decomposition goes hand in hand with the complex structure decomposition on the Riemann surface $\Sigma$, and may be achieved by introducing the standard complex frame basis $e^{a}=\left(e^{z}, e^{\bar{z}}\right)$, with metric $\delta_{z \bar{z}}=\delta_{\bar{z} z}=2$. The explicit expressions for the frame and flux fields in this basis may be deduced from

$$
\begin{array}{ll}
e^{z}=\left(e^{7}+i e^{8}\right) / 2 & e_{z}=e^{7}-i e^{8} \\
e^{\bar{z}}=\left(e^{7}-i e^{8}\right) / 2 & e_{\bar{z}}=e^{7}+i e^{8}
\end{array}
$$

Similar relations hold for the components of the fields $p_{a}, q_{a}, g_{a}$, so that, for example, $p_{z}=p_{7}-i p_{8}$, and $p_{\bar{z}}=p_{7}+i p_{8}$. The Pauli matrices in this basis take the form,

$$
\sigma^{z}=\left(\begin{array}{ll}
0 & 1 \\
0 & 0
\end{array}\right) \quad \sigma^{\bar{z}}=\left(\begin{array}{ll}
0 & 0 \\
1 & 0
\end{array}\right)
$$

We proceed to decompose the two-index spinor $\zeta$ into its two chirality components in this same 2-dimensional spinor basis,

$$
\zeta_{\eta_{1}, \eta_{2}}=\left(\begin{array}{c}
\tau^{(13)} \xi_{\eta_{1}, \eta_{2}}^{*} \\
\psi_{\eta_{1}, \eta_{2}}
\end{array}\right)
$$


where $\xi_{\eta_{1}, \eta_{2}}^{*}$ and $\psi_{\eta_{1}, \eta_{2}}$ are 1-component spinors. In this basis, the reduced BPS equations take the following form,

$$
\begin{aligned}
& \left(d_{1}\right) \quad 4 i p_{z} \xi+g_{z} \psi+g_{9} \tau^{(13)} \xi^{*}-h \tau^{(12)} \xi^{*}=0 \\
& \left(d_{2}\right) \quad 4 i p_{\bar{z}}^{*} \psi+g_{\bar{z}}^{*} \xi-g_{9}^{*} \tau^{(13)} \psi^{*}-h^{*} \tau^{(12)} \psi^{*}=0 \\
& \left(m_{1}\right) \quad \frac{i}{f_{5}} \tau^{(22)} \xi^{*}+\frac{D_{z} f_{5}}{f_{5}} \psi-f \tau^{(12)} \xi^{*}+\frac{i}{8}\left(-g_{z} \xi+g_{9} \tau^{(13)} \psi^{*}-h \tau^{(12)} \psi^{*}\right)=0 \\
& \left(m_{2}\right) \quad-\frac{i}{f_{5}} \tau^{(22)} \psi^{*}+\frac{D_{z} f_{5}}{f_{5}} \xi-f \tau^{(12)} \psi^{*}+\frac{i}{8}\left(-g_{\bar{z}}^{*} \psi-g_{9}^{*} \tau^{(13)} \xi^{*}-h^{*} \tau^{(12)} \xi^{*}\right)=0 \\
& \text { (i }) \quad \frac{i}{f_{2}} \tau^{(11)} \xi^{*}+\frac{D_{z} f_{2}}{f_{2}} \psi+f \tau^{(12)} \xi^{*}+\frac{i}{8}\left(3 g_{z} \xi-3 g_{9} \tau^{(13)} \psi^{*}-h \tau^{(12)} \psi^{*}\right)=0 \\
& \text { (i }) \quad \frac{i}{f_{2}} \tau^{(11)} \psi^{*}+\frac{D_{z} f_{2}}{f_{2}} \xi+f \tau^{(12)} \psi^{*}+\frac{i}{8}\left(3 g_{\bar{z}}^{*} \psi+3 g_{9}^{*} \tau^{(13)} \xi^{*}-h^{*} \tau^{(12)} \xi^{*}\right)=0 \\
& \left(9_{1}\right) \quad-\frac{\nu}{f_{1}} \tau^{(23)} \xi^{*}+\frac{D_{z} f_{1}}{f_{1}} \psi+f \tau^{(12)} \xi^{*}+\frac{i}{8}\left(-g_{z} \xi-3 g_{9} \tau^{(13)} \psi^{*}+3 h \tau^{(12)} \psi^{*}\right)=0 \\
& \left(9_{2}\right) \quad \frac{\nu}{f_{1}} \tau^{(23)} \psi^{*}+\frac{D_{z} f_{1}}{f_{1}} \xi+f \tau^{(12)} \psi^{*}+\frac{i}{8}\left(-g_{\bar{z}}^{*} \psi+3 g_{9}^{*} \tau^{(13)} \xi^{*}+3 h^{*} \tau^{(12)} \xi^{*}\right)=0 \\
& \left(+_{1}\right) \quad\left(D_{\bar{z}}-\frac{i}{2} \hat{\omega}_{\bar{z}}+\frac{i}{2} q_{\bar{z}}\right) \xi+\frac{i}{4} g_{z}^{*} \psi=0 \\
& \left(+_{2}\right) \quad\left(D_{z}-\frac{i}{2} \hat{\omega}_{z}-\frac{i}{2} q_{z}\right) \psi+f \tau^{(12)} \xi^{*}+\frac{i}{8}\left(g_{z} \xi+g_{9} \tau^{(13)} \psi^{*}+3 h \tau^{(12)} \psi^{*}\right)=0 \\
& \left(-_{1}\right) \quad\left(D_{z}-\frac{i}{2} \hat{\omega}_{z}+\frac{i}{2} q_{z}\right) \xi+f \tau^{(12)} \psi^{*}+\frac{i}{8}\left(g_{\bar{z}}^{*} \psi-g_{9}^{*} \tau^{(13)} \xi^{*}+3 h^{*} \tau^{(12)} \xi^{*}\right)=0 \\
& (-2) \quad\left(D_{\bar{z}}-\frac{i}{2} \hat{\omega}_{\bar{z}}-\frac{i}{2} q_{\bar{z}}\right) \psi+\frac{i}{4} g_{\bar{z}} \xi=0
\end{aligned}
$$

where $\hat{\omega}_{z}=i\left(\partial_{w} \rho\right) / \rho^{2}$. The action of the complex conjugation symmetry (3.23) is given by

$$
\begin{aligned}
\xi & \rightarrow \xi^{\prime}=e^{-i \theta} \tau^{(10)} \psi \\
\psi & \rightarrow \psi^{\prime}=e^{+i \theta} \tau^{(10)} \xi
\end{aligned}
$$

It acts by interchanging $\xi$ and $\psi$, together with the transformations on the bosonic fields, listed already in (3.19), and translated as follows in the chiral basis,

$$
\begin{array}{rlrl}
p_{z} & \rightarrow p_{z}^{\prime}=e^{4 i \theta}\left(p_{\bar{z}}\right)^{*} & g_{z} \rightarrow g_{z}^{\prime}=e^{2 i \theta}\left(g_{\bar{z}}\right)^{*} & h \rightarrow h^{\prime}=e^{2 i \theta} h^{*} \\
q_{z} \rightarrow q_{z}^{\prime}=-q_{z}+2 D_{z} \theta & g_{9} \rightarrow g_{9}^{\prime}=-e^{2 i \theta} g_{9}^{*} &
\end{array}
$$

This concludes the full reduction of the BPS equations onto the $A d S_{5} \times S^{2} \times S^{1} \times \Sigma$ Ansatz.

\section{Vanishing G implies the $A d S_{5} \times S^{5}$ solution}

In this section, we shall prove that, whenever the 3 -form flux field $G$ vanishes, the solution to the BPS equations is $A d S_{5} \times S^{5}$ itself. We shall also spell out the properties of this solution. 
When $G=0$, we have $g_{z}=g_{\bar{z}}=g_{9}=h=0$. For solutions with at least 16 supersymmetries, $\psi$ and $\xi$ cannot both vanish. The reduced dilatino equations (d) in (3.27) imply $p_{z}=p_{\bar{z}}=0$, so that $P=0$. By the Bianchi identities, we then have $d Q=0$. Using the $\mathrm{U}(1)_{q}$ gauge symmetry (3.15), we may now choose a gauge in which $Q=0$, so that,

$$
\begin{gathered}
g_{z}=g_{\bar{z}}=g_{9}=h=0 \\
p_{z}=p_{\bar{z}}=q_{z}=q_{\bar{z}}=0
\end{gathered}
$$

The gauge choice leaves open the symmetry of performing constant gauge transformations, which we shall fix later.

\subsection{Using the discrete symmetries of the reduced BPS equations}

Given the vanishing of the components of $G, P, Q$ in (4.1), all four generators $T_{1}, T_{2}, T_{3}$, and $T_{4}$ of (3.18) and (3.19) produce genuine discrete symmetries of (3.27). The generators $T_{1}, T_{2}$, and $T_{4}$ mutually commute and may be simultaneously diagonalized as follows,

$$
\begin{aligned}
& T_{1} \zeta=\tau^{(303)} \zeta=\nu \zeta \\
& T_{2} \zeta=\tau^{(300)} \zeta=\gamma \zeta \\
& T_{4} \zeta=e^{i \theta} \tau^{(030)} \sigma^{1} \zeta^{*}=\mu \zeta
\end{aligned}
$$

where $\nu, \gamma, \mu$ take on independently the values \pm 1 . The projection of $T_{1}$ has already been carried out in the previous section. Clearly, the sign $\mu$ in the $T_{3}$ projection can always be gauged away with a constant gauge transformation in $\mathrm{U}(1)_{q}$. Henceforth, we shall choose $\mu e^{i \theta}=1$. In terms of $\psi$ and $\xi$, these projections become,

$$
\begin{aligned}
& T_{2}: \quad \tau^{(30)} \psi=\gamma \psi \quad \tau^{(30)} \xi=-\gamma \xi \\
& T_{4}: \quad \xi=\tau^{(10)} \psi
\end{aligned}
$$

Solutions with opposite values of $\gamma$ are equivalent. Henceforth, we set $\gamma=+$, and shall treat $\psi$ and $\xi$ as two-component spinors, omitting their fixed $\eta_{1}$ indices.

$$
\begin{aligned}
& \psi=\left(\begin{array}{l}
\psi_{+} \\
\psi_{-}
\end{array}\right) \equiv\left(\begin{array}{l}
\psi_{++} \\
\psi_{+-}
\end{array}\right) \\
& \xi=\left(\begin{array}{l}
\xi_{+} \\
\xi_{-}
\end{array}\right) \equiv\left(\begin{array}{l}
\xi_{-+} \\
\xi_{--}
\end{array}\right)=\left(\begin{array}{l}
\psi_{++} \\
\psi_{+-}
\end{array}\right)
\end{aligned}
$$

After these simplifications, the remaining reduced BPS equations of (3.27) take the form,

$$
\begin{array}{ll}
(m) & \mp \frac{i}{f_{5}} \psi_{\mp}^{*}+\frac{D_{z} f_{5}}{f_{5}} \psi_{ \pm} \pm i f \psi_{\mp}^{*}=0 \\
(i) & \frac{i}{f_{2}} \psi_{\mp}^{*}+\frac{D_{z} f_{2}}{f_{2}} \psi_{ \pm} \mp i f \psi_{\mp}^{*}=0 \\
(9) \quad & \pm \frac{i \nu}{f_{1}} \psi_{ \pm}^{*}+\frac{D_{z} f_{1}}{f_{1}} \psi_{ \pm} \mp i f \psi_{\mp}^{*}=0 \\
(-) & \left(D_{\bar{z}}-\frac{i}{2} \hat{\omega}_{\bar{z}}\right) \psi_{ \pm}=0 \\
(+) & \left(D_{z}-\frac{i}{2} \hat{\omega}_{z}\right) \psi_{ \pm} \mp i f \psi_{\mp}^{*}=0
\end{array}
$$




\subsection{Generic solutions when $G=0$}

The $(m)$-equations may be expressed as follows,

$$
\frac{D_{z} f_{5}}{f_{5}} \psi_{ \pm}= \pm i\left(\frac{1}{f_{5}}-f\right) \psi_{\mp}^{*}
$$

The product of the + equation with the complex conjugate of the - equation gives,

$$
\left|\frac{D_{z} f_{5}}{f_{5}}\right|^{2} \psi_{+} \psi_{-}^{*}+\left(\frac{1}{f_{5}}-f\right)^{2} \psi_{+} \psi_{-}^{*}=0
$$

When $\psi_{+} \psi_{-}^{*} \neq 0,(4.7)$ implies $\partial_{z} f_{5}=0$ and $f f_{5}=1$. If $\psi_{+} \psi_{-}^{*}=0$, then either $\psi_{+}=0$ or $\psi_{-}^{*}=0$, but both cannot vanish simultaneously. In either case, (4.6) implies $\partial_{z} f_{5}=0$ and $f f_{5}=1$. Thus, $f$ and $f_{5}$ are constant satisfying $f f_{5}=1$.

- Next, using the expression $\hat{\omega}_{z}=i\left(\partial_{z} \rho\right) / \rho^{2}$, and $D_{z}=\rho^{-1} \partial_{z}$, we derive the complete solution to the (-) equations of (4.5), as follows,

$$
\psi_{+}=\sqrt{\rho} \alpha, \quad \psi_{-}=\sqrt{\rho} \beta \quad \partial_{\bar{z}} \alpha=\partial_{\bar{z}} \beta=0
$$

so that $\alpha$ and $\beta$ are holomorphic functions.

- From the differential equations $( \pm)$ in (4.5), we find $D_{z}\left(\left|\psi_{+}\right|^{2}+\left|\psi_{-}\right|^{2}\right)=0$. Therefore $\left|\psi_{+}\right|^{2}+\left|\psi_{-}\right|^{2}$ is a constant; we may normalize this to be $f_{5}$, which is also a constant.

$$
f_{5}=\left|\psi_{+}\right|^{2}+\left|\psi_{-}\right|^{2}=\rho(\alpha \bar{\alpha}+\beta \bar{\beta})
$$

- Using equation (4.9) to eliminate $f=f_{5}^{-1}$ from the (+) equation in (4.5), we find,

$$
\begin{aligned}
& \partial_{z}\left(\frac{\alpha}{\alpha \bar{\alpha}+\beta \bar{\beta}}\right)-\frac{i \bar{\beta}}{(\alpha \bar{\alpha}+\beta \bar{\beta})^{2}}=0 \\
& \partial_{z}\left(\frac{\beta}{\alpha \bar{\alpha}+\beta \bar{\beta}}\right)+\frac{i \bar{\alpha}}{(\alpha \bar{\alpha}+\beta \bar{\beta})^{2}}=0
\end{aligned}
$$

Multiplying the first by $\bar{\alpha}$, the second by $\bar{\beta}$, and adding gives an equation that is automatically satisfied. Thus, we may retain just the first equation. After some simplifications, it reduces to a holomorphic equation,

$$
\beta \partial_{z} \alpha-\alpha \partial_{z} \beta-i=0
$$

which may be easily solved, and gives the solution

$$
\beta(z)=-i \alpha(z) A(z) \quad A(z) \equiv \int_{z_{0}}^{z} \frac{d u}{\alpha(u)^{2}}
$$

with $z_{0}$ being the arbitrary integration constant. In fact, it will be convenient to express both $\alpha$ and $\beta$ solely in terms of the function $A$, namely,

$$
\alpha(z)=\frac{1}{\sqrt{\partial_{z} A(z)}} \quad \beta(z)=-\frac{i A(z)}{\sqrt{\partial_{z} A(z)}}
$$


To determine metric factors in terms of spinor components ${ }^{4}$ we make use of both algebraic and differential equations. On the one hand, from the (i) equations in (4.5), we have

$$
\frac{D_{z} f_{2}}{f_{2}}\left(\left|\psi_{+}\right|^{2}-\left|\psi_{-}\right|^{2}\right)=2 i f \psi_{-}^{*} \psi_{+}^{*}
$$

On the other hand, using differential equations $(+)$ and $(-)$, we obtain the relation

$$
D_{z}\left(\left|\psi_{+}\right|^{2}-\left|\psi_{-}\right|^{2}\right)=2 i f \psi_{-}^{*} \psi_{+}^{*}
$$

Combining the above equations gives,

$$
f_{2}=c_{2}\left(\left|\psi_{+}\right|^{2}-\left|\psi_{-}\right|^{2}\right)
$$

where $c_{2}$ is a constant. Similarly, from (9) equations, we may derive the following relations,

$$
\begin{gathered}
\frac{D_{z} f_{1}}{f_{1}}\left(\psi_{+}^{*} \psi_{-}+\psi_{-}^{*} \psi_{+}\right)=i f\left(\psi_{-}^{*} \psi_{-}-\psi_{+}^{*} \psi_{+}\right) \\
D_{z}\left(\psi_{+}^{*} \psi_{-}+\psi_{-}^{*} \psi_{+}\right)=i f\left(\psi_{-}^{*} \psi_{-}-\psi_{+}^{*} \psi_{+}\right)
\end{gathered}
$$

Combining these in turn implies,

$$
f_{1}=c_{1}\left(\psi_{+}^{*} \psi_{-}+\psi_{-}^{*} \psi_{+}\right)
$$

where $c_{1}$ is a constant.

- Using the expression for metric factors, $\alpha$ and $\beta$, it can be checked that (i) and (9) equations are all satisfied, if $c_{1}=c_{2}=1$. So all the reduced BPS equations are completely solved, and all fields are determined by a holomorphic function $A(z)$.

\subsection{Solution of $A d S_{5} \times S^{5}$}

Choosing $A(z)=-e^{-2 z}, f_{5}=1$, we recover the $A d S_{5} \times S^{5}$ solution,

$$
d s^{2}=d s_{A d S_{5}}^{2}+\left(\operatorname{th} x_{7}\right)^{2} d s_{S^{2}}^{2}+\frac{d x_{7}^{2}+d x_{8}^{2}}{\left(\operatorname{ch} x_{7}\right)^{2}}+\frac{\sin ^{2} x_{8} d x_{9}^{2}}{\left(\operatorname{ch} x_{7}\right)^{2}}
$$

To exhibit the metric in standard form, we change parametrization, $e^{x^{7}}=\tan \left(\frac{\theta}{2}\right)$,

$$
\begin{aligned}
d s^{2} & =d s_{A d S_{5}}^{2}+\cos ^{2} \theta d s_{S^{2}}^{2}+\sin ^{2} \theta\left(d x_{7}^{2}+d x_{8}^{2}\right)+\sin ^{2} \theta \sin ^{2} x_{8} d x_{9}^{2} \\
& =d s_{A d S_{5}}^{2}+\cos ^{2} \theta d s_{S^{2}}^{2}+d \theta^{2}+\sin ^{2} \theta d x_{8}^{2}+\sin ^{2} \theta \sin ^{2} x_{8} d x_{9}^{2} \\
& =d s_{A d S_{5}}^{2}+d \theta^{2}+\cos ^{2} \theta d s_{S^{2}}^{2}+\sin ^{2} \theta d s_{S^{2}}^{2}
\end{aligned}
$$

which is indeed $A d S_{5} \times S^{5}$. The solution to the spinor $\zeta$ is characterized by three projections,

$$
\begin{aligned}
\tau^{(030)} \sigma^{1} \zeta^{*} & =\zeta \\
\tau^{(303)} \zeta & =\nu \zeta \\
\tau^{(300)} \zeta & =\gamma \zeta
\end{aligned}
$$

\footnotetext{
${ }^{4} \mathrm{~A}$ complete analysis of Hermitian forms will be given in the next section.
} 
where we can have, independently, $\nu= \pm 1, \gamma= \pm 1$. In terms of functions $\zeta_{1}, \zeta_{2}$ defined below, the non-vanishing components of spinor solutions are (with $z=\left(x_{7}+i x_{8}\right) / 2$ ):

$$
\begin{aligned}
& \zeta_{1}=i^{\frac{1-\nu}{2}} i^{\frac{1-\gamma}{2}} \frac{e^{\bar{z}}}{\sqrt{2 \operatorname{ch} x_{7}}} \\
& \zeta_{2}=i^{\frac{\nu-1}{2}} i^{\frac{1+\gamma}{2}} \frac{e^{-\bar{z}}}{\sqrt{2 \operatorname{ch} x_{7}}} \\
& \nu=+1, \quad \gamma=+1 \quad \zeta_{+++}=\left(\begin{array}{l}
\zeta_{1} \\
\bar{\zeta}_{1}
\end{array}\right) \quad \zeta_{+-+}=\left(\begin{array}{c}
\zeta_{2} \\
-\bar{\zeta}_{2}
\end{array}\right) \\
& \nu=+1, \quad \gamma=-1 \quad \zeta_{-+-}=\left(\begin{array}{l}
\zeta_{1} \\
\bar{\zeta}_{1}
\end{array}\right) \quad \zeta_{---}=\left(\begin{array}{c}
\zeta_{2} \\
-\bar{\zeta}_{2}
\end{array}\right) \\
& \nu=-1, \quad \gamma=+1 \quad \zeta_{++-}=\left(\begin{array}{l}
\zeta_{1} \\
\bar{\zeta}_{1}
\end{array}\right) \quad \zeta_{+--}=\left(\begin{array}{c}
\zeta_{2} \\
-\bar{\zeta}_{2}
\end{array}\right) \\
& \nu=-1, \quad \gamma=-1 \quad \zeta_{-++}=\left(\begin{array}{l}
\zeta_{1} \\
\bar{\zeta}_{1}
\end{array}\right) \quad \zeta_{--+}=\left(\begin{array}{c}
\zeta_{2} \\
-\bar{\zeta}_{2}
\end{array}\right)
\end{aligned}
$$

With 8 independent solutions to killing spinors $\chi$ and 4 independent solutions to $\zeta$, we indeed recover 32 supersymmetries as expected.

\section{$5 \quad$ Metric factors in terms of spinor bilinears}

The metric factors $f_{1}, f_{2}, f_{5}$ may be related to bilinear (or more accurately Hermitian forms) of the spinors $\psi, \xi$. Since the metric factors $f_{1}, f_{2}, f_{5}$ are real, the spinor bilinears need to be real, and invariant under $\mathrm{U}(1)_{q}$ transformations. These requirements rule out combinations of the form $\psi^{t} \tau^{(\alpha \beta)} \psi, \xi^{t} \tau^{(\alpha \beta)} \xi$, and $\psi^{t} \tau^{(\alpha \beta)} \xi$, because they are complex, and $\psi^{\dagger} \tau^{(\alpha \beta)} \xi$, and $\xi^{\dagger} \tau^{(\alpha \beta)} \psi$ because they fail to be $\mathrm{U}(1)_{q}$-invariant. Thus, the only real and $\mathrm{U}(1)_{q}$-invariant combinations left are $\psi^{\dagger} \tau^{(\alpha \beta)} \psi$ and $\xi^{\dagger} \tau^{(\alpha \beta)} \xi$.

\subsection{Example of metric factor calculation}

The detailed calculation is relegated to appendix $\mathrm{D}$, here, we shall illustrate the procedure by giving an specific example of finding spinor bilinears of $f_{5}$.

We shall use combinations of the differential equations $( \pm)$ in (3.28) and of the algebraic gravitino BPS equations to bring out the corresponding relations. To this end, we compute,

$$
\begin{aligned}
D_{z}\left(\psi^{\dagger} \psi\right) & =-f \psi^{\dagger} \tau^{(12)} \xi^{*}+\frac{i}{4} g_{\bar{z}}^{*} \xi^{\dagger} \psi-\frac{i}{8}\left(g_{z} \psi^{\dagger} \xi+g_{9} \psi^{\dagger} \tau^{(13)} \psi^{*}+3 h \psi^{\dagger} \tau^{(12)} \psi^{*}\right) \\
D_{z}\left(\xi^{\dagger} \xi\right) & =+f \psi^{\dagger} \tau^{(12)} \xi^{*}+\frac{i}{4} g_{z} \psi^{\dagger} \xi+\frac{i}{8}\left(-g_{\bar{z}}^{*} \xi^{\dagger} \psi+g_{9}^{*} \xi^{\dagger} \tau^{(13)} \xi^{*}-3 h^{*} \xi^{\dagger} \tau^{(12)} \xi^{*}\right)
\end{aligned}
$$

Then, left-multiplying equation $\left(m_{1}\right)$ by $\psi^{\dagger}$, and left-multiplying the complex conjugate of equation $\left(m_{2}\right)$ by $\xi^{\dagger}$, we find,

$$
\begin{aligned}
& \frac{D_{z} f_{5}}{f_{5}}\left(\psi^{\dagger} \psi\right)=-\frac{i}{f_{5}} \psi^{\dagger} \tau^{(22)} \xi^{*}+f \psi^{\dagger} \tau^{(12)} \xi^{*}+\frac{i}{8}\left(g_{z} \psi^{\dagger} \xi-g_{9} \psi^{\dagger} \tau^{(13)} \psi^{*}+h \psi^{\dagger} \tau^{(12)} \psi^{*}\right) \\
& \frac{D_{z} f_{5}}{f_{5}}\left(\xi^{\dagger} \xi\right)=+\frac{i}{f_{5}} \xi^{\dagger} \tau^{(22)} \psi^{*}+f \xi^{\dagger} \tau^{(12)} \psi^{*}+\frac{i}{8}\left(g_{\bar{z}}^{*} \xi^{\dagger} \psi+g_{9}^{*} \xi^{\dagger} \tau^{(13)} \xi^{*}+h^{*} \xi^{\dagger} \tau^{(12)} \xi^{*}\right)
\end{aligned}
$$


With appropriate combinations of the above equations, all terms would cancel out. We may get the following relation,

$$
D_{z}\left(\psi^{\dagger} \psi+\xi^{\dagger} \xi\right)-\frac{D_{z} f_{5}}{f_{5}}\left(\psi^{\dagger} \psi+\xi^{\dagger} \xi\right)=0
$$

Which further implies, $D_{z}\left[f_{5}^{-1}\left(\psi^{\dagger} \psi+\xi^{\dagger} \xi\right)\right]=0$, so that $f_{5}$ may be expressed as the product of constant $C_{5}^{(00)}$ and spinor bilinears,

$$
f_{5}^{-1}\left(\psi^{\dagger} \psi+\xi^{\dagger} \xi\right)=C_{5}^{(00)}
$$

\subsection{Summary of metric factor expressions}

As a summary, we list all the generic relations, valid for arbitrary values of all the fields of the supergravity Ansatz. For notational simplicity of this and the remaining sections, we shall use the following definition for Hermitian forms.

$$
H_{ \pm}^{(\alpha \beta)} \equiv \psi^{\dagger} \tau^{(\alpha \beta)} \psi \pm \xi^{\dagger} \tau^{(\alpha \beta)} \xi
$$

In terms of these Hermitian composites, we have the following result,

$$
\begin{array}{rlrl}
f_{5}^{-1} H_{+}^{(\alpha \beta)} & =C_{5}^{(\alpha \beta)} & & \tau^{(\alpha \beta)} \in\left\{\tau^{(00)}, \tau^{(31)}, \tau^{(32)}, \tau^{(33)}\right\} \\
f_{5}^{3} H_{-}^{(\alpha \beta)} & =C_{5}^{(\alpha \beta)} & \tau^{(\alpha \beta)} \in\left\{\tau^{(01)}, \tau^{(30)}\right\} \\
f_{2}^{-1} H_{-}^{(33)} & =C_{2}^{(33)} & & \\
f_{2}^{1 / 3} H_{+}^{(\alpha \beta)} & =C_{2}^{(\alpha \beta)} & \tau^{(\alpha \beta)} & \in\left\{\tau^{(31)}, \tau^{(32)}\right\} \\
f_{1}^{-1} H_{+}^{(\alpha \beta)} & =C_{1}^{(\alpha \beta)} & \tau^{(\alpha \beta)} \in\left\{\tau^{(01)}, \tau^{(30)}, \tau^{(32)}, \tau^{(33)}\right\}
\end{array}
$$

When $g_{9}=0$, we have additional non-generic relations, which are summarized below,

$$
\begin{aligned}
f_{5}^{3} H_{-}^{(\alpha \beta)} & =C_{5}^{(\alpha \beta)} & & \tau^{(\alpha \beta)} \in\left\{\tau^{(02)}, \tau^{(03)}\right\} \\
f_{2}^{-1} H_{-}^{(\alpha \beta)} & =C_{2}^{(\alpha \beta)} & & \tau^{(\alpha \beta)} \in\left\{\tau^{(00)}, \tau^{(10)}, \tau^{(20)}\right\} \\
f_{1}^{-1} H_{+}^{(\alpha \beta)} & =C_{1}^{(\alpha \beta)} & & \tau^{(\alpha \beta)} \in\left\{\tau^{(10)}, \tau^{(12)}, \tau^{(13)}, \tau^{(20)}, \tau^{(22)}, \tau^{(23)}\right\}
\end{aligned}
$$

When $h=0$, the additional relations are,

$$
\begin{aligned}
f_{2}^{-1} H_{-}^{(\alpha \beta)} & =C_{2}^{(\alpha \beta)} & & \tau^{(\alpha \beta)} \in\left\{\tau^{(01)}, \tau^{(11)}, \tau^{(21)}\right\} \\
f_{1}^{3} H_{-}^{(\alpha \beta)} & =C_{1}^{(\alpha \beta)} & & \tau^{(\alpha \beta)} \in\left\{\tau^{(11)}, \tau^{(21)}\right\}
\end{aligned}
$$

If both $h=g_{9}=0$, there are some additional relations besides all the above ones,

$$
\begin{aligned}
f_{2}^{-1} H_{-}^{(\alpha \beta)} & =C_{2}^{(\alpha \beta)} & & \tau^{(\alpha \beta)} \in\left\{\tau^{(02)}, \tau^{(12)}, \tau\right. \\
f_{1}^{3} H_{-}^{(\alpha \beta)} & =C_{1}^{(\alpha \beta)} & & \tau^{(\alpha \beta)} \in\left\{\tau^{(02)}, \tau^{(03)}\right\}
\end{aligned}
$$

Note that, by linearity of the BPS equation, and the fact that $f_{5}$ and $\psi^{\dagger} \psi+\xi^{\dagger} \xi$ must be positive, we may rescale both $\xi$ and $\psi$ by the same real constant, and without loss of generality choose the following normalization,

$$
H_{+}^{(00)}=f_{5}
$$

In the next section, we shall show that similar unique correspondences exist for $f_{1}$ and $f_{2}$. 


\section{Vanishing Hermitian forms}

In the preceding section, it was shown that certain Hermitian forms of the supersymmetry spinors are related to the metric factors $f_{1}, f_{2}, f_{5}$, in terms of unknown constants. In the present section, we shall show that certain Hermitian forms vanish automatically as a result of the BPS equations. These relations will be obtained using the reality properties of various combinations. Their derivations will involve combinations in which the quantities $p_{z}, p_{\bar{z}}^{*}, g_{z}$, and $g_{\bar{z}}^{*}$ are algebraically eliminated, since these functions do not, generically, obey definite reality conditions. In this section, we shall use the following definition for Hermtian forms,

$$
\begin{aligned}
& H_{ \pm}^{(\alpha \beta)} \equiv \psi^{\dagger} \tau^{(\alpha \beta)} \psi \pm \xi^{\dagger} \tau^{(\alpha \beta)} \xi \\
& H_{g \pm}^{(\alpha \beta)} \equiv g_{9} \psi^{\dagger} \tau^{(\alpha \beta)} \xi \pm g_{9}^{*} \xi^{\dagger} \tau^{(\alpha \beta)} \psi \\
& H_{h \pm}^{(\alpha \beta)} \equiv h \psi^{\dagger} \tau^{(\alpha \beta)} \xi \pm h^{*} \xi^{\dagger} \tau^{(\alpha \beta)} \psi
\end{aligned}
$$

where the first line reproduces the definition already used in (5.5). Note that $H_{ \pm}^{(\alpha \beta)}, H_{g+}^{(\alpha \beta)}$, and $H_{h+}^{(\alpha \beta)}$ are real, while $H_{g-}^{(\alpha \beta)}$, and $H_{h-}^{(\alpha \beta)}$ are purely imaginary.

\subsection{Example of Hermitian relation calculation}

We leave the detailed calculation in appendix E, and quote the results below. There are three different ways to compute vanishing hermitian forms, each will provide additional relations among Hermitian forms, which are also summarized below.

Before giving the complete list of all Hermitian relations, we illustrate one example about computing Hermitian forms.

Considering a combination of the BPS equations of (3.27), given by $2(\mathrm{~m})+(\mathrm{i})+(9)$. All $f, g_{z}, g_{\bar{z}}, h, h^{*}$ terms cancel in these combinations, and the resulting equations are,

$$
\begin{aligned}
D_{z} \ln \left(f_{5}^{2} f_{2} f_{1}\right) \psi+\left(\frac{2 i}{f_{5}} \tau^{(22)}+\frac{i}{f_{2}} \tau^{(11)}-\frac{\nu}{f_{1}} \tau^{(23)}\right) \xi^{*}-\frac{i}{2} g_{9} \tau^{(13)} \psi^{*}=0 \\
D_{z} \ln \left(f_{5}^{2} f_{2} f_{1}\right) \xi+\left(-\frac{2 i}{f_{5}} \tau^{(22)}+\frac{i}{f_{2}} \tau^{(11)}+\frac{\nu}{f_{1}} \tau^{(23)}\right) \psi^{*}+\frac{i}{2} g_{9}^{*} \tau^{(13)} \xi^{*}=0
\end{aligned}
$$

Multiplying the first equation by $\xi^{t}$, the second by $-\psi^{t}$, adding both to cancel the differential terms, and taking the transpose, we obtain,

$$
\begin{aligned}
0= & \xi^{\dagger}\left(\frac{2 i}{f_{5}} \tau^{(22)}+\frac{i}{f_{2}} \tau^{(11)}+\frac{\nu}{f_{1}} \tau^{(23)}\right) \xi-\frac{i}{2} g_{9} \psi^{\dagger} \tau^{(13)} \xi \\
& +\psi^{\dagger}\left(\frac{2 i}{f_{5}} \tau^{(22)}-\frac{i}{f_{2}} \tau^{(11)}+\frac{\nu}{f_{1}} \tau^{(23)}\right) \psi-\frac{i}{2} g_{9}^{*} \xi^{\dagger} \tau^{(13)} \psi
\end{aligned}
$$

Each term in $\xi^{\dagger} \tau \xi$ or $\psi^{\dagger} \tau \psi$, and each combination of terms involving $g_{9}$ and $g_{9}^{*}$, is either real or purely imaginary. Separating real and imaginary parts of the above equations, we have,

$$
H_{+}^{(23)}=0 \quad \frac{2}{f_{5}} H_{+}^{(22)}-\frac{1}{f_{2}} H_{-}^{(11)}-\frac{1}{2} H_{g+}^{(13)}=0
$$

So we obtained one vanishing Hermitian form, and a relation among them. 


\subsection{Summary of all Hermitian relations}

As a summary, we list all the vanishing Hermitian relations below,

$$
\begin{array}{ll}
H_{+}^{(\alpha \beta)}=0 \quad(\alpha \beta) \in\{(10),(11),(13),(20),(21),(23),(30),(31),(32),(33)\} \\
H_{-}^{(\alpha \beta)}=0 \quad(\alpha \beta) \in\{(00),(01),(02),(03),(12),(22)\} \\
H_{g+}^{(\alpha \beta)}=0 \quad(\alpha \beta) \in\{(00),(02),(03),(10),(12),(20),(22)\} \\
H_{g-}^{(\alpha \beta)}=0 \quad(\alpha \beta) \in\{(01),(30),(32),(33)\} \\
H_{h+}^{(\alpha \beta)}=0 \quad(\alpha \beta) \in\{(00),(31),(32),(33)\} \\
H_{h-}^{(\alpha \beta)}=0 \quad(\alpha \beta) \in\{(01),(02),(03),(12),(22)\}
\end{array}
$$

The remaining equations from the first set are as follows,

$$
\begin{aligned}
& \text { (00) } \frac{2}{f_{5}} H_{+}^{(22)}-\frac{1}{f_{2}} H_{-}^{(11)}-\frac{1}{2} H_{g+}^{(13)}=0 \\
& \text { (01) } \frac{1}{f_{2}} H_{-}^{(10)}-\frac{\nu}{f_{1}} H_{+}^{(22)}=0 \\
& \text { (02) } \frac{2}{f_{5}} H_{-}^{(20)}-\frac{\nu}{f_{1}} H_{-}^{(21)}-\frac{i}{2} H_{g-}^{(11)}=0 \\
& \text { (12) } \frac{2}{f_{5}} H_{-}^{(30)}+\frac{1}{f_{2}} H_{+}^{(03)}-\frac{\nu}{f_{1}} H_{-}^{(31)}=0 \\
& \text { (22) } \frac{2}{f_{5}} H_{+}^{(00)}+\frac{1}{f_{2}} H_{-}^{(33)}-\frac{\nu}{f_{1}} H_{+}^{(01)}-\frac{1}{2} H_{g+}^{(31)}=0 \\
& \text { (30) } \frac{2}{f_{5}} H_{+}^{(12)}+\frac{1}{f_{2}} H_{-}^{(21)}+\frac{1}{2} H_{g+}^{(23)}=0 \\
& \text { (31) } \frac{1}{f_{2}} H_{-}^{(20)}+\frac{\nu}{f_{1}} H_{+}^{(12)}=0 \\
& \text { (32) } \frac{2}{f_{5}} H_{-}^{(10)}-\frac{\nu}{f_{1}} H_{-}^{(11)}+\frac{i}{2} H_{g-}^{(21)}=0
\end{aligned}
$$

Those of the second set are as follows,

$$
\begin{aligned}
& \text { (02) } \frac{i}{f_{5}} H_{-}^{(20)}-\frac{1}{8} H_{g-}^{(11)}-\frac{i}{8} H_{h+}^{(10)}=0 \\
& \text { (12) } \frac{1}{f_{5}} H_{-}^{(30)}-f H_{+}^{(00)}=0 \\
& \text { (32) } \frac{1}{f_{5}} H_{-}^{(10)}-\frac{i}{8} H_{g-}^{(21)}+\frac{1}{8} H_{h+}^{(20)}=0 \\
& \text { (02) } 3 H_{g-}^{(11)}-i H_{h+}^{(10)}=0 \\
& \text { (12) } \frac{1}{f_{2}} H_{+}^{(03)}+f H_{+}^{(00)}=0 \\
& \text { (32) } 3 i H_{g-}^{(21)}+H_{h+}^{(20)}=0
\end{aligned}
$$


Finally, those of the third set are given by,

$$
\begin{aligned}
& \text { (00) } \frac{1}{f_{5}} H_{+}^{(22)}+\frac{1}{2} H_{g+}^{(13)}=0 \\
& \text { (01) } \frac{i \nu}{f_{1}} H_{+}^{(22)}-2 \text { if } H_{-}^{(13)}-\frac{1}{2} H_{h-}^{(13)}=0 \\
& \text { (03) } 2 \text { if } H_{-}^{(11)}+\frac{1}{2} H_{h-}^{(11)}=0 \\
& \text { (22) } \frac{i}{f_{5}} H_{+}^{(00)}+\frac{i \nu}{f_{1}} H_{+}^{(01)}-2 i f H_{-}^{(30)}+\frac{i}{2} H_{g+}^{(31)}-\frac{1}{2} H_{h-}^{(30)}=0 \\
& \text { (30) } \frac{1}{f_{5}} H_{+}^{(12)}-\frac{1}{2} H_{g+}^{(23)}=0 \\
& \text { (31) } \frac{\nu}{f_{1}} H_{+}^{(12)}+2 f H_{-}^{(23)}-\frac{i}{2} H_{h-}^{(23)}=0 \\
& \text { (33) } 2 f H_{-}^{(21)}-\frac{i}{2} H_{h-}^{(21)}=0
\end{aligned}
$$

\subsection{Implications for the metric factors}

The relations between the metric factors $f_{5}, f_{2}, f_{1}$ and Hermitian forms, derived in (5.6), together with the vanishing Hermitian forms of the preceding section, produce vanishing value for the following constants,

$$
\begin{aligned}
& 0=C_{5}^{(31)}=C_{5}^{(32)}=C_{5}^{(33)}=C_{5}^{(01)} \\
& 0=C_{2}^{(31)}=C_{2}^{(32)} \\
& 0=C_{1}^{(30)}=C_{1}^{(32)}=C_{1}^{(33)}
\end{aligned}
$$

The remaining non-vanishing Hermitian forms $f_{1}, f_{2}, f_{5}$ are given as follows,

$$
\begin{aligned}
& f_{5}^{-1} H_{+}^{(00)}=1 \quad f_{5}^{3} H_{-}^{(30)}=c_{5} \\
& f_{2}^{-1} H_{-}^{(33)}=c_{2} \\
& f_{1}^{-1} H_{+}^{(01)}=c_{1}
\end{aligned}
$$

Here, we have used the normalization $C_{5}^{(00)}=1$ derived in (5.10), and abbreviated $C_{1}^{(01)}$, $C_{2}^{(33)}$, and $C_{5}^{(30)}$ by $c_{1}, c_{2}$, and $c_{5}$ respectively, all three of which are real constants.

A series of additional constant Hermitian forms were derived in (5.7) and (5.8) respectively when $g_{9}=0$ and $h=0$ or both. These relations are readily seen to follow directly from the equations (6.6), (6.7), and (6.8), and may thus be omitted.

\section{General solution to the reduced BPS equations}

In this section, we shall exploit the vanishing hermitian forms to solve the BPS equations, and conclude that the only solution to the reduced BPS equations is $A d S_{5} \times S^{5}$. 


\subsection{Solving the Hermitian relations $H_{ \pm}^{(\alpha \beta)}=0$}

We begin by solving the Hermitian relations of the form $H_{ \pm}^{(\alpha \beta)}=0$, namely those relations given in the first two lines of (6.5). Once we shall have solved for those, we shall solve the remaining Hermitian equations of (6.5) as well as the remaining BPS equations.

Every set of four Hermitian relations can be grouped together to derive an equivalent set of simplified relations between spinor components,

$$
\begin{aligned}
& H_{-}^{(00)}=H_{-}^{(03)}=H_{+}^{(30)}=H_{+}^{(33)}=0 \quad \Rightarrow \quad \xi_{+ \pm}^{*} \xi_{+ \pm}-\psi_{- \pm}^{*} \psi_{- \pm}=0 \\
& \xi_{- \pm}^{*} \xi_{- \pm}-\psi_{+ \pm}^{*} \psi_{+ \pm}=0 \\
& H_{-}^{(01)}=H_{-}^{(02)}=H_{+}^{(31)}=H_{+}^{(32)}=0 \quad \Rightarrow \quad \xi_{+ \pm}^{*} \xi_{+\mp}-\psi_{- \pm}^{*} \psi_{-\mp}=0 \\
& \xi_{- \pm}^{*} \xi_{-\mp}-\psi_{+ \pm}^{*} \psi_{+\mp}=0 \\
& H_{+}^{(10)}=H_{+}^{(13)}=H_{+}^{(20)}=H_{+}^{(23)}=0 \quad \Rightarrow \quad \xi_{ \pm+}^{*} \xi_{\mp+}+\psi_{ \pm+}^{*} \psi_{\mp+}=0 \\
& \xi_{ \pm-}^{*} \xi_{\mp-}+\psi_{ \pm-}^{*} \psi_{\mp-}=0 \\
& H_{+}^{(11)}=H_{-}^{(12)}=H_{+}^{(21)}=H_{-}^{(22)}=0 \quad \Rightarrow \quad \xi_{+ \pm}^{*} \xi_{-\mp}+\psi_{+\mp}^{*} \psi_{- \pm}=0 \\
& \xi_{- \pm}^{*} \xi_{+\mp}+\psi_{-\mp}^{*} \psi_{+ \pm}=0
\end{aligned}
$$

From the first group (7.1), we deduce that $\left|\xi_{+ \pm}\right|=\left|\psi_{- \pm}\right|,\left|\xi_{- \pm}\right|=\left|\psi_{+ \pm}\right|$. Thus, the corresponding functions are related by phases, which we introduce as follows,

$$
\begin{array}{lll}
\xi_{++}=e^{i \theta_{1}} \psi_{-+} & \xi_{+-}=e^{i \theta_{2}} \psi_{--} \\
\xi_{-+}=e^{i \theta_{3}} \psi_{++} & \xi_{--}=e^{i \theta_{4}} \psi_{+-}
\end{array}
$$

Substituting these results into the second group (7.2), we obtain,

$$
\begin{aligned}
& \left(e^{i\left(\theta_{2}-\theta_{1}\right)}-1\right) \psi_{-+}^{*} \psi_{--}=0 \\
& \left(e^{i\left(\theta_{4}-\theta_{3}\right)}-1\right) \psi_{++}^{*} \psi_{+-}=0
\end{aligned}
$$

while from the third and fourth groups (7.3) and (7.4), we obtain,

$$
\begin{aligned}
& e^{i\left(\theta_{3}-\theta_{1}\right)} \psi_{-+}^{*} \psi_{++}+\psi_{++}^{*} \psi_{-+}=0 \\
& e^{i\left(\theta_{4}-\theta_{2}\right)} \psi_{--}^{*} \psi_{+-}+\psi_{+-}^{*} \psi_{--}=0 \\
& e^{i\left(\theta_{4}-\theta_{1}\right)} \psi_{-+}^{*} \psi_{+-}+\psi_{+-}^{*} \psi_{-+}=0 \\
& e^{i\left(\theta_{3}-\theta_{2}\right)} \psi_{--}^{*} \psi_{++}+\psi_{++}^{*} \psi_{--}=0
\end{aligned}
$$

The solution depends upon whether the spinor components $\psi_{ \pm \pm}$generically vanish or not.

\subsubsection{The generic case for which $\psi_{++} \psi_{+_{-}} \psi_{-+} \psi_{--} \neq 0$}

When the components $\psi_{++}, \psi_{+-}, \psi_{-+}$, and $\psi_{--}$are all generic and non-vanishing, then equations (7.6) require,

$$
\theta_{1}=\theta_{2} \quad \theta_{3}=\theta_{4}
$$


We introduce the angles $\Theta$ and $\Theta^{\prime}$, defined by,

$$
\begin{aligned}
4 \Theta & \equiv \theta_{1}-\theta_{3}+\pi & \theta_{1} & =+2 \Theta+2 \Theta^{\prime} \\
4 \Theta^{\prime} & \equiv \theta_{1}+\theta_{3}-\pi & \theta_{3} & =-2 \Theta+2 \Theta^{\prime}+\pi
\end{aligned}
$$

Note that we also have $4 \Theta=\theta_{2}-\theta_{3}+\pi=\theta_{1}-\theta_{4}+\pi=\theta_{2}-\theta_{4}+\pi$ in view of (7.8). The equations of (7.7) amount to relations between the phases of the components of $\psi$ which may all be expressed in terms of the angle $\Theta$ as follows,

$$
\begin{array}{ll}
\frac{\psi_{-+}^{*}}{\psi_{-+}}=e^{4 i \Theta} \frac{\psi_{++}^{*}}{\psi_{++}} & \frac{\psi_{--}^{*}}{\psi_{--}}=e^{4 i \Theta} \frac{\psi_{+-}^{*}}{\psi_{+-}} \\
\frac{\psi_{-+}^{*}}{\psi_{-+}}=e^{4 i \Theta} \frac{\psi_{+-}^{*}}{\psi_{+-}} & \frac{\psi_{--}^{*}}{\psi_{--}}=e^{4 i \Theta} \frac{\psi_{++}^{*}}{\psi_{++}}
\end{array}
$$

or equivalently,

$$
\frac{\psi_{++}^{*}}{\psi_{++}}=\frac{\psi_{+-}^{*}}{\psi_{+-}} \quad \frac{\psi_{-+}^{*}}{\psi_{-+}}=\frac{\psi_{--}^{*}}{\psi_{--}} \quad \frac{\psi_{-+}^{*}}{\psi_{-+}}=\frac{\left(e^{-2 i \Theta} \psi_{++}\right)^{*}}{e^{-2 i \Theta} \psi_{++}}
$$

The solutions to these relations may be parametrized in terms of 4 real arbitrary functions $r_{++}, r_{+-}, r_{-+}, r_{--}$, the angle $\Theta$, and a further arbitrary angle $\Lambda$,

$$
\begin{aligned}
& \psi_{++}=r_{++} e^{i \Lambda+i \Theta} \\
& \psi_{+-}=r_{+-} e^{i \Lambda+i \Theta} \\
& \psi_{-+}=r_{-+} e^{i \Lambda-i \Theta} \\
& \psi_{--}=r_{--} e^{i \Lambda-i \Theta}
\end{aligned}
$$

The solutions for $\xi$ now follow immediately,

$$
\begin{aligned}
& \xi_{++}=r_{-+} e^{i \Lambda^{\prime}+i \Theta} \\
& \xi_{+-}=r_{--} e^{i \Lambda^{\prime}+i \Theta} \\
& \xi_{-+}=-r_{++} e^{i \Lambda^{\prime}-i \Theta} \\
& \xi_{--}=-r_{+-} e^{i \Lambda^{\prime}-i \Theta}
\end{aligned}
$$

where $\Lambda^{\prime}=2 \Theta^{\prime}+\Lambda$. (Using the U(1) gauge freedom, we could remove the overall relative phase factor between $\psi$ and $\xi$, namely $\Lambda^{\prime}-\Lambda$, and set this angle to zero so that $\Lambda^{\prime}=\Lambda$. To keep symmetry manifest, and to remain as general as possible, we shall not make this gauge choice here.) One is then left with 4 real functions $r$, and two angles $\Theta$, and $\Lambda$.

\subsubsection{Non-generic cases with vanishing spinor components}

It is clear from the above generic solution that a non-generic solution could be viewed as the limit of the generic case. For example, if $\psi_{--}=0$, then this case can be viewed as the limit in which $r_{--} \rightarrow 0$. Tracking this case back to the original equations, it is clear that one retains the first and third equations of (7.11), whose solution simply amounts to the generic solution in which we set $r_{--}=0$. 
One can check that the only exceptions to the generic solution is when either $\psi_{-+}=$ $\psi_{--}=0, \psi_{++} \psi_{+-} \neq 0$ or $\psi_{++}=\psi_{+-}=0, \psi_{-+} \psi_{--} \neq 0$, which has equivalent derivations. We shall focus on the first possibility then, which may be parameterized by four real functions $r_{++}, r_{+-}, \Lambda_{1}, \Lambda_{2}$, and equations (7.6), (7.7) are automatically satisfied.

$$
\begin{aligned}
\theta_{3} & =\theta_{4} \\
\psi_{++} & =r_{++} e^{i \Lambda_{1}}=r_{1} e^{i \Lambda_{1}} \\
\psi_{+-} & =r_{+-} e^{i \Lambda_{2}}=r_{2} e^{i \Lambda_{2}} \\
\xi_{-+} & =r_{1} e^{i\left(\Lambda_{1}+\theta_{3}\right)} \\
\xi_{--} & =r_{2} e^{i\left(\Lambda_{2}+\theta_{3}\right)}
\end{aligned}
$$

We shall call this the second type of solution, the difference from the generic solution being $\psi_{++}$and $\psi_{+-}$may have different phases.

\subsubsection{Two types of solutions}

The solutions of (7.12) and (7.13) constitute the first type of solution. They may be expressed in matrix form, as follows,

$$
\begin{aligned}
\psi & =e^{i \Lambda+i \Theta \tau^{(30)}} r \\
\xi & =\left(i \tau^{(20)}\right) e^{i \Lambda^{\prime}-i \Theta \tau^{(30)}} r
\end{aligned}
$$

where $r$ is given by,

$$
r=\left(\begin{array}{c}
r_{++} \\
r_{+-} \\
r_{-+} \\
r_{--}
\end{array}\right) \equiv\left(\begin{array}{l}
r_{1} \\
r_{2} \\
r_{3} \\
r_{4}
\end{array}\right)
$$

We can readily check that this form of $\psi$ and $\xi$ indeed reproduces all the vanishing Hermitian forms of the first two lines of (6.5). In checking this, the dependences on the angles $\Lambda$ and $\Lambda^{\prime}$ clearly drop out, but the dependence on $\Theta$ may or may not drop out. We find,

$$
H_{ \pm}^{(\alpha \beta)}=r^{t} e^{-i \Theta \tau^{(30)}}\left[\tau^{(\alpha \beta)} \pm \tau^{(20)}\left(\tau^{(\alpha \beta)}\right)^{t} \tau^{(20)}\right] e^{i \Theta \tau^{(30)}} r
$$

This combination will vanish for a given assignment of $(\alpha \beta)$ and all values of $r$ and $\Theta$ provided either $(i)$ the terms in the square brackets cancel; or $(i i) \tau^{(\alpha \beta)}$ is anti-symmetric and commutes with $\tau^{(30)}$ (which is the case precisely only for $\tau^{(02)}$ and $\tau^{(32)}$ ). These two vanishing conditions reproduce all the vanishing Hermitian forms of the first two lines of (6.5), as well as two additional relations,

$$
H_{+}^{(02)}=H_{-}^{(32)}=0
$$

These relations do not figure amongst the first two lines of (6.5), and remarkably never enter the additional relations either. Equation (7.14) gives the second type of solution, 
with non-vanishing components,

$$
\begin{aligned}
& \psi_{++}=r_{1} e^{i \Lambda_{1}} \\
& \psi_{+-}=r_{2} e^{i \Lambda_{2}} \\
& \xi_{-+}=r_{1} e^{i\left(\Lambda_{1}+\theta_{3}\right)} \\
& \xi_{--}=r_{2} e^{i\left(\Lambda_{2}+\theta_{3}\right)}
\end{aligned}
$$

\subsection{Solving the Hermitian relations $H_{g \pm}^{(\alpha \beta)}$}

\subsubsection{For the first type of solution}

We shall focus on the first type of generic solution (7.15) in the section. Expressing $H_{g \pm}^{(\alpha \beta)}$ in terms of the above parametrization gives

$$
\begin{aligned}
H_{g \pm}^{(\alpha \beta)}= & i g_{9} e^{i\left(\Lambda^{\prime}-\Lambda\right)} r^{t} e^{-i \Theta \tau^{(30)} \tau^{(\alpha \beta)} \tau^{(20)} e^{-i \Theta \tau^{(30)}} r} \\
& \mp i g_{9}^{*} e^{-i\left(\Lambda^{\prime}-\Lambda\right)} r^{t} e^{i \Theta \tau^{(30)}} \tau^{(20)} \tau^{(\alpha \beta)} e^{i \Theta \tau^{(30)}} r
\end{aligned}
$$

This form may be simplified according to whether $\tau^{(\alpha \beta)}$ commutes or anti-commutes with $\tau^{(30)}$, so that the formula may be split into two cases,

$$
\begin{aligned}
& \alpha=0,3 \quad H_{g \pm}^{(\alpha \beta)}=i g_{9} e^{i\left(\Lambda^{\prime}-\Lambda\right)} r^{t} \tau^{(\alpha \beta)} \tau^{(20)} r \\
& \mp i g_{9}^{*} e^{-i\left(\Lambda^{\prime}-\Lambda\right)} r^{t} \tau^{(20)} \tau^{(\alpha \beta)} r \\
& \alpha=1,2 \\
& H_{g \pm}^{(\alpha \beta)}=i g_{9} e^{i\left(\Lambda^{\prime}-\Lambda\right)} r^{t} e^{-2 i \Theta \tau^{(30)}} \tau^{(\alpha \beta)} \tau^{(20)} r \\
& \mp i g_{9}^{*} e^{-i\left(\Lambda^{\prime}-\Lambda\right)} r^{t} e^{2 i \Theta \tau^{(30)}} \tau^{(20)} \tau^{(\alpha \beta)} r
\end{aligned}
$$

It follows from the vanishing of $r^{t} \tau^{(\gamma \delta)} r$, when $\tau^{(\gamma \delta)}$ is anti-symmetric, that we have the following automatic cancelations,

$$
H_{g+}^{(00)}=H_{g+}^{(03)}=H_{g+}^{(12)}=H_{g+}^{(22)}=H_{g-}^{(01)}=H_{g-}^{(32)}=0
$$

The remaining cases of the middle two lines of (6.5), impose the following conditions. For $H_{g-}^{(30)}, H_{g-}^{(33)}$, and $H_{g+}^{(02)}$, we have,

$$
\left(g_{9} e^{i\left(\Lambda^{\prime}-\Lambda\right)}-g_{9}^{*} e^{-i\left(\Lambda^{\prime}-\Lambda\right)}\right) r^{t} \tau^{(\gamma \delta)} r=0 \quad(\gamma \delta) \in\{(10),(13),(22)\}
$$

while the vanishing of $H_{g+}^{(10)}$, and $H_{g+}^{(20)}$, imposes the following relations,

$$
\left(\begin{array}{cc}
i \sin (2 \Theta) \mathcal{G}_{-} & -\cos (2 \Theta) \mathcal{G}_{+} \\
i \cos (2 \Theta) \mathcal{G}_{-} & \sin (2 \Theta) \mathcal{G}_{+}
\end{array}\right)\left(\begin{array}{c}
r^{t} r \\
r^{t} \tau^{(30)} r
\end{array}\right)=0
$$

where $\mathcal{G}_{ \pm} \equiv g_{9} e^{i\left(\Lambda^{\prime}-\Lambda\right)} \pm g_{9}^{*} e^{-i\left(\Lambda^{\prime}-\Lambda\right)}$. Since $r^{t} r \neq 0$, this equation must admit non-trivial solutions, requiring that the determinant of the above $2 \times 2$ matrix vanish, i.e. $\mathcal{G}_{+} \mathcal{G}_{-}=0$. It is manifest that $\mathcal{G}_{+}=0$, with $\mathcal{G}_{-} \neq 0$, cannot be a viable solution. Hence, we must have

$$
\mathcal{G}_{-}=g_{9} e^{i\left(\Lambda^{\prime}-\Lambda\right)}-g_{9}^{*} e^{-i\left(\Lambda^{\prime}-\Lambda\right)}=0
$$

which then automatically solves also the equations (7.23).

Now if $g_{9} \neq 0$, we have $\mathcal{G}_{+} \neq 0$, and also the relation $r^{t} \tau^{(30)} r=0$.

$$
r_{1}^{2}+r_{2}^{2}=r_{3}^{2}+r_{4}^{2}
$$




\subsubsection{Vanishing of $g_{9}$ for first type of solution}

The above result contradicts the equation (12) in (6.7), which implies,

$$
H_{+}^{(00)}=\frac{1}{f f_{5}} H_{-}^{(30)}=\frac{2}{f f_{5}}\left(r_{1}^{2}+r_{2}^{2}-r_{3}^{2}-r_{4}^{2}\right)=0
$$

which is impossible since $H_{+}^{(00)}$ is positive-definite, and proportional to $f_{5}$. As a result, we may conclude that $g_{9}=0$. Now, by rechecking equations $(6.6),(6.7),(6.8)$ using the condition $g_{9}=0$, we may find some additional vanishing Hermitian forms.

$$
\begin{aligned}
& H_{-}^{(10)}=H_{-}^{(11)}=H_{+}^{(12)} H_{-}^{(20)}=H_{-}^{(21)}=H_{+}^{(22)}=0 \\
& H_{h+}^{(10)}=H_{h-}^{(11)}=H_{h+}^{(20)}=H_{h-}^{(21)}=0
\end{aligned}
$$

Combing with the original vanishing Hermitian forms, we obtain

$$
\psi^{\dagger} \tau^{(\alpha \beta)} \psi=0 \quad(\alpha \beta) \in\{10,11,12,20,21,22\}
$$

The (11),(12),(21),(22) equations are equivalent to the following ones,

$$
\psi_{ \pm+}^{*} \psi_{\mp-}=0
$$

from which we know that

$$
r_{1} r_{4}=r_{2} r_{3}=0
$$

From the (10) and (20) equations, we also derive that

$$
r_{1} r_{3}+r_{2} r_{4}=0
$$

\subsubsection{Vanishing of $g_{9}$ for second type of solution}

The computation of $H_{g \pm}^{(\alpha \beta)}$ equations is a lot simpler for the second type of solution. Consider $H_{g+}^{(10)}=H_{g+}^{(20)}=0$, using the parametrization (7.19), two equations combine to be,

$$
g_{9}\left(r_{1}^{2}+r_{2}^{2}\right)=0
$$

So we immediately have $g_{9}=0$.

\subsection{Non-existence of solutions except for $A d S_{5} \times S^{5}$}

\subsubsection{First type of solution}

Without loss of generality, let's choose $r_{4}=0$ in equations (7.31) and (7.32). It then follows that either $r_{1}=r_{2}=0$ or $r_{3}=0$. We now examine the dilatino equations in (3.27),

$$
\begin{array}{ll}
\left(d_{1}\right) & 4 i p_{z} \xi+g_{z} \psi-h \tau^{(12)} \xi^{*}=0 \\
\left(d_{2}\right) & 4 i p_{\bar{z}}^{*} \psi+g_{\bar{z}}^{*} \xi-h^{*} \tau^{(12)} \psi^{*}=0
\end{array}
$$

If $r_{1}=r_{2}=r_{4}=0$, to have a non-vanishing value for $r_{3}$, we must have

$$
p_{z}=p_{\bar{z}}=g_{z}=g_{\bar{z}}=h=0
$$


From the analysis in section 4 , we know that this leads to the $A d S_{5} \times S^{5}$ solution. On the other hand, if $r_{3}=r_{4}=0$, the first dilatino equation reads in component form as,

$$
\begin{array}{ll}
0=p_{z} \xi_{- \pm}=p_{\bar{z}}^{*} \psi_{+ \pm} & \\
0=g_{z} \psi_{++}+i h \psi_{+-}^{*} e^{-i \theta_{3}} & 0=i h^{*} \psi_{++} e^{i \theta_{3}}+g_{z}^{*} \psi_{+-}^{*} \\
0=g_{\bar{z}}^{*} e^{i \theta_{3}} \psi_{++}+i h^{*} \psi_{+-}^{*} & 0=i h \psi_{++}+g_{\bar{z}} e^{-i \theta_{3}} \psi_{+-}^{*}
\end{array}
$$

In order to have non-vanishing spinor solutions, we should require

$$
\begin{aligned}
p_{z}=p_{\bar{z}} & =0 \\
|h|^{2}+\left|g_{z}\right|^{2} & =0 \\
|h|^{2}+\left|g_{\bar{z}}\right|^{2} & =0
\end{aligned}
$$

The second dilatino equation provides similar results, requiring $p_{z}=p_{\bar{z}}=g_{z}=g_{\bar{z}}=h=0$. The solution is still limited to $A d S_{5} \times S^{5}$.

\subsubsection{Second type of solution}

The analysis is identical to the one used for the first type of solution, and we again reach the conclusion that the only possible solution is $A d S_{5} \times S^{5}$.

\section{Ansatz with $\mathrm{SO}(2,4) \times \mathrm{SO}(4)$ symmetry}

Another realization of the superalgebra $\mathrm{SU}(2,2 \mid 2)$ in Type IIB supergravity is motivated by the system of a stack of D3-branes in the presence of probe D7-branes (see the Introduction). This realization requires extending the invariance by adjoining a purely bosonic $\mathrm{SU}(2)$ group, so that we have full $\mathrm{SU}(2,2 \mid 2) \times \mathrm{SU}(2)$ symmetry [23]. Its bosonic symmetry is $\mathrm{SO}(2,4) \times \mathrm{SO}(4) \times \mathrm{SO}(2)$. In this section, we shall use a less restrictive bosonic symmetry $\mathrm{SO}(2,4) \times \mathrm{SO}(4)$, thereby increasing the generality of our results. We shall show that the only solution is $A d S_{5} \times S^{5}$ for this realization of the symmetry.

\subsection{Construction of the Ansatz}

We seek the most general Ansatz in Type IIB supergravity with $\mathrm{SO}(2,4) \times \mathrm{SO}(4)$ symmetry. The $\mathrm{SO}(2,4)$-factor requires the geometry to contain an $A d S_{5}$-factor, while the $\mathrm{SO}(4)$ factor requires an $S^{3}$-factor, both of which are warped over a 2-dimensional surface $\Sigma$ with boundary. The total space-time then has the structure $A d S_{5} \times S^{3} \times \Sigma$. Following a construction similar to the one given in section 2, the Ansatz for the metric is found to be,

$$
d s^{2}=f_{5}^{2} d s_{A d S_{5}}^{2}+f_{3}^{2} d s_{S^{3}}^{2}+d s_{\Sigma}^{2}
$$

where $f_{3}$ and $f_{5}$ are functions on $\Sigma$ only. Orthonormal frames are defined by.

$$
\begin{array}{rlrl}
e^{m} & =f_{5} \hat{e}^{m} & m & =0,1,2,3,4 \\
e^{i} & =f_{3} \hat{e}^{i} & i & =5,6,7 \\
e^{a} & a & =8,9
\end{array}
$$


Here, the metrics $d s_{A d S_{5}}^{2}$ and $d s_{S^{3}}^{2}$, as well as the orthonormal frames $\hat{e}^{m}$ and $\hat{e}^{i}$, refer to the spaces $A d S_{5}$ and $S^{3}$ with unit radius. In particular, we have

$$
\begin{aligned}
d s_{A d S_{5}}^{2} & =\eta_{m n} \hat{e}^{m} \otimes \hat{e}^{n} \\
d s_{S^{3}}^{2} & =\delta_{i j} \hat{e}^{i} \otimes \hat{e}^{j} \\
d s_{\Sigma}^{2} & =\delta_{a b} e^{a} \otimes e^{b}
\end{aligned}
$$

The dilaton/axion field $B$ is a function of $\Sigma$ only, and the associated composites $P, Q$ of (2.1) are decomposed as follows, $P=p_{a} e^{a}$, and $Q=q_{a} e^{a}$, where $p_{a}$ and $q_{a}$ are again functions of $\Sigma$ only. Finally, the most general Ansätze for the 3 - and 5-form fields $G$ and $F_{(5)}$ consistent with $\mathrm{SO}(2,4) \times \mathrm{SO}(4)$ invariance, are given by

$$
\begin{aligned}
F_{(5)} & =f\left(e^{01234}-e^{56789}\right) \\
G & =i g e^{567}
\end{aligned}
$$

By $\mathrm{SO}(2,4) \times \mathrm{SO}(4)$-invariance, the coefficient functions $p_{a}, q_{a}, g$ and $f$ depend only on $\Sigma$. The functions $f, q_{a}$ are real, while $p_{a}, g$ are complex-valued.

\subsection{Reducing the BPS equations}

The supersymmetry spinor $\varepsilon$ must be covariant under $\mathrm{SO}(2,4) \times \mathrm{SO}(4)$, and may be built from the Killing spinors on the space $A d S_{5} \times S^{3}$, where each factor space has unit radius.

\subsubsection{Killing spinors}

We begin by constructing suitable Killing spinors on $A d S_{5} \times S^{3}$. We define a spinor representation $\chi^{\eta_{1}, \eta_{2}}$ of $\mathrm{SO}(2,4) \times \mathrm{SO}(4)$ by the following equations [10, 13],

$$
\begin{array}{rlrl}
\left(\hat{\nabla}_{m}-\frac{1}{2} \eta_{1} \gamma_{m} \otimes I_{2}\right) \chi^{\eta_{1}, \eta_{2}} & =0 & & m=0,1,2,3,4 \\
\left(\hat{\nabla}_{i}-\frac{i}{2} \eta_{2} I_{4} \otimes \gamma_{i}\right) \chi^{\eta_{1}, \eta_{2}} & =0 & i & =5,6,7
\end{array}
$$

Here, $\gamma_{m}$ and $\gamma_{i}$ are the Dirac matrices on $A d S_{5}$ and $S^{3}$ respectively (see appendix A.2). Integrability requires $\eta_{1}^{2}=\eta_{2}^{2}=1$. The respective solution spaces are of dimension 4 and 2 , so that $\chi^{\eta_{1}, \eta_{2}}$ has 8 independent solutions. Charge conjugation $\chi \rightarrow \chi^{c}$ is given by,

$$
\left(\chi^{c}\right)^{\eta_{1}, \eta_{2}}=\left(B_{(1)} \otimes B_{(2)}\right)^{-1}\left(\chi^{\eta_{1}, \eta_{2}}\right)^{*}
$$

and reverses the sign of $\eta_{1}$, so that $\left(\chi^{c}\right)^{\eta_{1}, \eta_{2}}$ is proportional to $\chi^{-\eta_{1}, \eta_{2}}$. The overall proportionality factor may be chosen freely; here, we shall use the following convention,

$$
\left(\chi^{c}\right)^{\eta_{1}, \eta_{2}}=\chi^{-\eta_{1}, \eta_{2}}
$$

Putting all together, the full 32-component spinor $\varepsilon$ may thus be decomposed in terms of Killing spinors on $A d S_{5} \times S^{3}$ as follows,

$$
\varepsilon=\sum_{\eta_{1}, \eta_{2}} \chi^{\eta_{1}, \eta_{2}} \otimes\left(\zeta_{\eta_{1}, \eta_{2}} \otimes u_{+}+\zeta_{\eta_{1}, \eta_{2}}^{\prime} \otimes u_{-}\right)
$$


We use the $u_{ \pm}$basis of (3.7), so that $\zeta_{\eta_{1}, \eta_{2}}$ and $\zeta_{\eta_{1}, \eta_{2}}^{\prime}$ are independent 2-dimensional spinor functions on $\Sigma$. The factor in parentheses in (8.8) parametrizes a general 4-component spinor. The 10-dimensional chirality condition $\Gamma^{11} \varepsilon=-\varepsilon$, with $\Gamma^{11}=I_{4} \otimes I_{2} \otimes \sigma_{3} \otimes \sigma_{2}$, reduces to $\zeta_{\eta_{1}, \eta_{2}}^{\prime}=-i \sigma^{3} \zeta_{\eta_{1}, \eta_{2}}$. so that the spinor $\varepsilon$ takes the following form,

$$
\varepsilon=\sum_{\eta_{1}, \eta_{2}} \chi^{\eta_{1}, \eta_{2}} \otimes\left(\zeta_{\eta_{1}, \eta_{2}} \otimes u_{+}-i \sigma^{3} \zeta_{\eta_{1}, \eta_{2}} \otimes u_{-}\right)
$$

We shall continue to use the $\tau$ matrix notation as introduced in equation (3.12).

\subsubsection{The reduced BPS equations}

The detailed calculations for the reduction of the BPS equations are in appendix C. Here, we summarize the final results. The dilatino BPS equation is given by,

$$
\text { (d) } \quad 0=g \zeta=p_{a} \sigma^{a} \sigma^{1} \zeta^{*}
$$

Any solution with non-vanishing $\zeta$ requires $g=0$. The second equation $\operatorname{implies} \operatorname{det}\left(p_{a} \sigma^{a}\right)=$ 0 , from which we derive either $p_{8}=i p_{9} \neq 0$ or $p_{8}=-i p_{9} \neq 0$ or $p_{8}=p_{9}=0$. The gravitino equation decomposes into a system of 3 equations,

$$
\begin{aligned}
\text { (m) } & 0 & =\left(\frac{1}{2 f_{5}} \tau^{(30)}-\frac{1}{2} \frac{D_{a} f_{5}}{f_{5}} \sigma^{a} \sigma^{3}-\frac{f}{2}\right) \zeta \\
(i) & 0 & =\left(\frac{1}{2 f_{3}} \tau^{(03)} \sigma^{3}-\frac{1}{2} \frac{D_{a} f_{3}}{f_{3}} \sigma^{a} \sigma^{3}+\frac{f}{2}\right) \zeta \\
\text { (a) } & 0 & =\left(D_{a}+\frac{i}{2} \hat{\omega}_{a} \sigma^{3}+\frac{f}{2} \sigma_{a} \sigma^{3}-\frac{i}{2} q_{a}\right) \zeta
\end{aligned}
$$

\subsubsection{The chiral form of the reduced BPS equations}

Using the complex basis defined in (3.24), we proceed to decompose the two-index spinor $\zeta$ into its two chirality components in this same 2-dimensional spinor basis,

$$
\zeta_{\eta_{1}, \eta_{2}}=\left(\begin{array}{c}
\xi_{\eta_{1}, \eta_{2}} \\
\psi_{\eta_{1}, \eta_{2}}
\end{array}\right)
$$

The reduced gravitino BPS equations now becomes

$$
\begin{array}{ll}
\left(m_{1}\right) \quad\left(\frac{1}{2 f_{5}} \tau^{(30)}-\frac{f}{2}\right) \xi+\frac{D_{z} f_{5}}{2 f_{5}} \psi=0 \\
\left(m_{2}\right) \quad-\frac{D_{\bar{z}} f_{5}}{2 f_{5}} \xi+\left(\frac{1}{2 f_{5}} \tau^{(30)}-\frac{f}{2}\right) \psi=0 \\
\left(i_{1}\right) \quad\left(\frac{1}{2 f_{3}} \tau^{(03)}+\frac{f}{2}\right) \xi+\frac{D_{z} f_{3}}{2 f_{3}} \psi=0 \\
\left(i_{2}\right) \quad-\frac{D_{\bar{z}} f_{3}}{2 f_{3}} \xi+\left(-\frac{1}{2 f_{3}} \tau^{(03)}+\frac{f}{2}\right) \psi=0 \\
\left(-_{1}\right) \quad\left(D_{z}+\frac{i}{2} \hat{\omega}_{z}-\frac{i}{2} q_{z}\right) \xi=0
\end{array}
$$




$$
\begin{array}{ll}
\left(-_{2}\right) & \left(D_{z}-\frac{i}{2} \hat{\omega}_{z}-\frac{i}{2} q_{z}\right) \psi+f \xi=0 \\
\left(+_{1}\right) \quad & \left(D_{\bar{z}}+\frac{i}{2} \hat{\omega}_{\bar{z}}-\frac{i}{2} q_{\bar{z}}\right) \xi-f \psi=0 \\
\left(+_{2}\right) \quad & \left(D_{\bar{z}}-\frac{i}{2} \hat{\omega}_{\bar{z}}-\frac{i}{2} q_{\bar{z}}\right) \psi=0
\end{array}
$$

\subsection{Solving the reduced BPS equations}

From the argument given below (8.10), we have three possible solutions for $p_{a}$. We shall now show that only the $p_{a}=0$ solution is viable. Indeed, suppose that we had a solution with $p_{8}=i p_{9} \neq 0$. From (8.10), this implies that $\psi=0$. Then from $\left(-_{2}\right)$ in (8.13), we find $f=0$ in order to have non-zero $\xi$. But now the $\left(m_{1}\right)$ equation becomes $\frac{1}{2 f_{5}} \tau^{(30)} \xi=0$, which in turn implies $\xi=0$, making the resulting solution trivial. The same arguments eliminates the case $p_{8}=-i p_{9} \neq 0$. Thus we are left with the only remaining solution $p_{8}=p_{9}=0$. Now when $p_{a}=0$, the Bianchi identity $d Q=0$ implies that $q_{a}$ are constants. With the help of the $\mathrm{U}(1)_{q}$ gauge symmetry, we may choose the gauge $q_{a}=0$.

\subsubsection{Using discrete symmetries}

There are two commuting discrete symmetries for the reduced BPS equations,

$$
\begin{aligned}
& T_{1}: \zeta \rightarrow \tau^{(30)} \zeta \\
& T_{2}: \zeta \rightarrow \tau^{(03)} \zeta
\end{aligned}
$$

so we can project the solution to the eigenspace of $\tau^{(30)}$ and $\tau^{(03)}$,

$$
\begin{aligned}
& \tau^{(30)} \zeta=\nu \zeta \\
& \tau^{(03)} \zeta=\gamma \zeta
\end{aligned}
$$

It turns out that different projections will give the same solution for supergravity fields. We restrict to the projection with $\nu=\gamma=1$, so that only the $\zeta_{++}$component can be non-zero; we shall abbreviate this component simply as $\zeta$.

\subsubsection{Hermitian forms for metric factors}

The calculations of Hermitian forms for metric factors proceed in the same way as for $\mathrm{SO}(2,4) \times \mathrm{SO}(3) \times \mathrm{SO}(2)$ case, and we will omit some details in the calculation. Using differential equations $(-)(+)$ in equation $(8.13)$, we find,

$$
D_{z}(\bar{\psi} \psi+\bar{\xi} \xi)=0
$$

So $\bar{\psi} \psi+\bar{\xi} \xi$ is a constant, which may be normalized to $f_{5}$,

$$
f_{5}=\bar{\psi} \psi+\bar{\xi} \xi
$$

since $f_{5}$ is a constant too, which is derived from $(m)$ equation in the next subsection. Using $(+)$ and $(-)$ and $(i)$ equations, we find $D_{z}\left(f_{3}^{-1}(\bar{\psi} \psi-\bar{\xi} \xi)\right)=0$, whose general solution is 
given by, $f_{3}=c_{3}(\bar{\psi} \psi-\bar{\xi} \xi)$. Using the fact that $f f_{5}=1$ and the $(i)$ equation, the normalization factor $c_{3}$ is determined to be 1 . So the expression for $f_{3}$ becomes,

$$
f_{3}=\bar{\psi} \psi-\bar{\xi} \xi
$$

\subsubsection{Generic solution to reduced BPS equations}

From equations $\left(m_{1}\right)$ and $\left(m_{2}\right)$, we have,

$$
\left(\frac{1}{2 f_{5}}-\frac{f}{2}\right)^{2} \psi+\left|\frac{D_{z} f_{5}}{2 f_{5}}\right|^{2} \psi=0
$$

the solution to which is,

$$
D_{z} f_{5}=0 \quad f f_{5}=1
$$

which imply that $f$ and $f_{5}$ are constants. The solution to $\left(-{ }_{1}\right)$ and $\left(+_{2}\right)$ is given by,

$$
\begin{aligned}
\xi & =\rho^{1 / 2} \overline{\alpha(z)} \\
\psi & =\rho^{1 / 2} \beta(z)
\end{aligned}
$$

Where $\alpha(z), \beta(z)$ are holomorphic functions. Using the above expression, the $\left(-{ }_{2}\right)$ and $\left(+_{1}\right)$ equations now become,

$$
\begin{array}{ll}
\left(-_{2}\right) & \partial_{z}(\rho \beta)+f \rho^{2} \bar{\alpha}=0 \\
\left(+_{1}\right) & \partial_{z}(\rho \alpha)-f \rho^{2} \bar{\beta}=0
\end{array}
$$

From the Hermitian form for $f_{5}$ in equation (8.17), we derive $\rho=f_{5} /\left(|\alpha|^{2}+|\beta|^{2}\right)$, and the differential equations become

$$
\begin{array}{ll}
\left(-_{2}\right) & \partial_{z}\left(\frac{\beta}{|\alpha|^{2}+|\beta|^{2}}\right)+\frac{\bar{\alpha}}{\left(|\alpha|^{2}+|\beta|^{2}\right)^{2}}=0 \\
\left(+{ }_{1}\right) & \partial_{z}\left(\frac{\alpha}{|\alpha|^{2}+|\beta|^{2}}\right)-\frac{\bar{\beta}}{\left(|\alpha|^{2}+|\beta|^{2}\right)^{2}}=0
\end{array}
$$

These equations may be compared to equations (4.10), which differ only by a factor of $i$. Let $A(z)$ be an arbitrary holomorphic function, the solution is

$$
\alpha(z)=\frac{1}{\sqrt{\partial_{z} A(z)}} \quad \beta(z)=-\frac{A(z)}{\sqrt{\partial_{z} A(z)}}
$$

\subsubsection{Uniqueness of the $A d S_{5} \times S^{5}$}

The above solution coincides with $A d S_{5} \times S^{5}$. This may be seen by a convenient choice of the coordinate $z$, for which $A(z)=\left(e^{z}-i e^{-z}\right) /\left(e^{z}+i e^{-z}\right)$, for which the metric manifestly factorizes and agrees with the unit radius metric on $A d S_{5} \times S^{5}$. Because there are 8 solutions to the Killing spinor $\chi^{\eta_{1} \eta_{2}}$, as well as 4 solutions for spinor $\zeta$ from the different projections, we recover indeed 32 supersymmetries. 


\section{A Clifford algebras}

\section{A.1 Clifford algebra basis for $A d S_{5} \times S^{2} \times \Sigma \times S^{1}$ space}

We choose a basis for the Clifford algebra which is well-adapted to the $A d S_{5} \times S^{2} \times \Sigma \times S^{1}$, with the frame labeled as in (2.17),

$$
\begin{array}{rlrl}
\Gamma^{m} & =\gamma^{m} \otimes I_{2} \otimes I_{2} \otimes \sigma^{3} & m & =0,1,2,3,4 \\
\Gamma^{i} & =I_{4} \otimes \gamma^{i} \otimes I_{2} \otimes \sigma^{1} & i & =5,6 \\
\Gamma^{a} & =I_{4} \otimes \sigma^{3} \otimes \gamma^{a} \otimes \sigma^{1} & & a=7,8 \\
\Gamma^{9} & =I_{4} \otimes \sigma^{3} \otimes \gamma^{9} \otimes \sigma^{1} & &
\end{array}
$$

where a convenient basis for the lower dimensional Clifford algebras is as follows,

$$
\begin{array}{rlrl}
i \gamma^{0} & =\sigma^{2} \otimes I_{2} & & \gamma^{5}=\gamma^{7}=\sigma^{1} \\
\gamma^{1} & =\sigma^{1} \otimes I_{2} & \gamma^{6}=\gamma^{8}=\sigma^{2} \\
\gamma^{2} & =\sigma^{3} \otimes \sigma^{2} & & \gamma^{9}=\sigma^{3} \\
\gamma^{3} & =\sigma^{3} \otimes \sigma^{1} & & \\
\gamma^{4} & =\sigma^{3} \otimes \sigma^{3} & &
\end{array}
$$

We also find the following matrix

$$
\begin{array}{rlrl}
\gamma^{01234} & =i I_{4} & \Gamma^{01234} & =i I_{4} \otimes I_{2} \otimes I_{2} \otimes \sigma_{3} \\
\gamma_{(2)}=-i \gamma^{56} & =\sigma^{3} \\
\gamma_{(3)}=-i \gamma^{78} & =\sigma^{3} & \Gamma_{(2)} & =-i \Gamma^{56}=I_{4} \otimes \sigma^{3} \otimes I_{2} \otimes I_{2} \\
\Gamma_{(3)} & =-i \Gamma^{78}=I_{4} \otimes I_{2} \otimes \sigma^{3} \otimes I_{2}
\end{array}
$$

The 10-dimensional chirality matrix in this basis is given by

$$
\Gamma^{11}=\Gamma^{0123456789}=I_{4} \otimes I_{2} \otimes I_{2} \otimes \sigma_{2}
$$

The complex conjugation matrices in each subspace are defined by

$$
\begin{array}{lll}
\left(\gamma^{m}\right)^{*}=-B_{(1)} \gamma^{m} B_{(1)}^{-1} & \left(B_{(1)}\right)^{*} B_{(1)}=-I_{2} & B_{(1)}=\sigma^{3} \otimes \sigma^{2} \\
\left(\gamma^{i}\right)^{*}=+B_{(2)} \gamma^{i} B_{(2)}^{-1} & \left(B_{(2)}\right)^{*} B_{(2)}=+I_{2} & B_{(2)}=\gamma^{5}=\sigma^{1} \\
\left(\gamma^{a}\right)^{*}=-B_{(3)} \gamma^{a} B_{(3)}^{-1} & \left(B_{(3)}\right)^{*} B_{(3)}=-I_{2} & B_{(3)}=\gamma^{8}=\sigma^{2} \\
\left(\gamma^{9}\right)^{*}=-B_{(4)} \gamma^{9} B_{(4)}^{-1} & \left(B_{(4)}\right)^{*} B_{(4)}=-I_{2} & B_{(4)}=\gamma^{8}=\sigma^{2}
\end{array}
$$

where in the last column we have also listed the form of these matrices in our particular basis. The 10-dimensional complex conjugation matrix $\mathcal{B}$ is defined by $\left(\Gamma^{M}\right)^{*}=\mathcal{B} \Gamma^{M} \mathcal{B}^{-1}$ and $\mathcal{B B}^{*}=I$, and in this basis is given by

$$
\mathcal{B}=B_{(1)} \otimes B_{(2)} \otimes B_{(3)} \otimes \sigma^{1}=\sigma^{3} \otimes \sigma^{2} \otimes \sigma^{1} \otimes \sigma^{2} \otimes \sigma^{1}
$$




\section{A.2 Clifford algebra basis for $A d S_{5} \times S^{3} \times \Sigma$ space}

We choose another basis of the Clifford algebra for this ansatz, which is well-adapted to the $A d S_{5} \times S^{3}$, with the frame labeled as in (8.2),

$$
\begin{array}{rlrl}
\Gamma^{m} & =\gamma^{m} \otimes I_{2} \otimes I_{2} \otimes \sigma^{3} & m & =0,1,2,3,4 \\
\Gamma^{i} & =I_{4} \otimes \gamma^{i} \otimes I_{2} \otimes \sigma^{1} & i & =5,6,7 \\
\Gamma^{a} & =I_{4} \otimes I_{2} \otimes \gamma^{a} \otimes \sigma^{2} & a & =8,9
\end{array}
$$

where a convenient basis for the lower dimensional Clifford algebras is as follows,

$$
\begin{array}{rlrl}
i \gamma^{0} & =\sigma^{2} \otimes I_{2} & \gamma^{5}=\gamma^{8}=\sigma^{1} \\
\gamma^{1} & =\sigma^{1} \otimes I_{2} & \gamma^{6}=\gamma^{9}=\sigma^{2} \\
\gamma^{2} & =\sigma^{3} \otimes \sigma^{2} & \gamma^{7}=\sigma^{3} & \\
\gamma^{3} & =\sigma^{3} \otimes \sigma^{1} & & \\
\gamma^{4} & =\sigma^{3} \otimes \sigma^{3} & &
\end{array}
$$

We also find the following matrix

$$
\begin{aligned}
\gamma^{01234} & =i I_{4} & \Gamma^{01234} & =i I_{4} \otimes I_{2} \otimes I_{2} \otimes \sigma_{3} \\
\gamma_{\Sigma}=-i \gamma^{89} & =\sigma^{3} & \Gamma_{\Sigma} & =-i \Gamma^{89}=I_{4} \otimes I_{2} \otimes \sigma_{3} \otimes I_{2}
\end{aligned}
$$

The 10-dimensional chirality matrix in this basis is given by

$$
\Gamma^{11}=\Gamma^{0123456789}=I_{4} \otimes I_{2} \otimes \sigma_{3} \otimes \sigma_{2}
$$

The complex conjugation matrices in each subspace are defined by

$$
\begin{array}{lll}
\left(\gamma^{m}\right)^{*}=-B_{(1)} \gamma^{m} B_{(1)}^{-1} & \left(B_{(1)}\right)^{*} B_{(1)}=-I_{2} & B_{(1)}=\sigma^{3} \otimes \sigma^{2} \\
\left(\gamma^{i}\right)^{*}=-B_{(2)} \gamma^{i} B_{(2)}^{-1} & \left(B_{(2)}\right)^{*} B_{(2)}=-I_{2} & B_{(2)}=\gamma^{6}=\sigma^{2} \\
\left(\gamma^{a}\right)^{*}=-B_{(3)} \gamma^{a} B_{(3)}^{-1} & \left(B_{(3)}\right)^{*} B_{(3)}=-I_{2} & B_{(3)}=\gamma^{9}=\sigma^{2}
\end{array}
$$

where in the last column we have also listed the form of these matrices in our particular basis. The 10-dimensional complex conjugation matrix $\mathcal{B}$ is defined by $\left(\Gamma^{M}\right)^{*}=\mathcal{B} \Gamma^{M} \mathcal{B}^{-1}$ and $\mathcal{B B}^{*}=I$, and in this basis is given by

$$
\mathcal{B}=B_{(1)} \otimes B_{(2)} \otimes B_{(3)} \otimes \sigma^{2}=\sigma^{3} \otimes \sigma^{2} \otimes \sigma^{2} \otimes \sigma^{2} \otimes \sigma^{2}
$$

\section{B Reducing the BPS equations: $A d S_{5} \times S^{2} \times \Sigma \times S^{1}$ case}

In this appendix, we shall present the detailed calculations required for the reduction of the BPS equations in $A d S_{5} \times S^{2} \times \Sigma \times S^{1}$ case. The reduction of the BPS equations (2.11) to our Ansatz requires the following combinations,

$$
\begin{aligned}
P \cdot \Gamma & =p_{a} \Gamma^{a} \\
G \cdot \Gamma & =6\left(i g_{a} \Gamma^{56 a}+i g_{9} \Gamma^{569}+h \Gamma^{789}\right) \\
F_{(5)} \cdot \Gamma & =240 f \Gamma^{01234}
\end{aligned}
$$


The corresponding $\Gamma$-matrices, in the basis given in appendix A.1, are given by,

$$
\begin{aligned}
\Gamma^{a} & =I_{4} \otimes \sigma^{3} \otimes \gamma^{a} \otimes \sigma^{1} \\
\Gamma^{56 \tilde{a}} & =i I_{4} \otimes I_{2} \otimes \gamma^{\tilde{a}} \otimes \sigma^{1} \\
\Gamma^{789} & =i I_{4} \otimes \sigma^{3} \otimes I_{2} \otimes \sigma^{1} \\
\Gamma^{01234} & =i I_{4} \otimes I_{2} \otimes I_{2} \otimes \sigma^{3}
\end{aligned}
$$

We shall also need the decompositions of $\mathcal{B}^{-1} \varepsilon^{*}$,

$$
\mathcal{B}^{-1} \varepsilon^{*}=\sum_{\eta_{1}, \eta_{2}, \eta_{3}} \chi^{\eta_{1}, \eta_{2}} \chi^{\eta_{3}} \otimes * \zeta_{\eta_{1}, \eta_{2}, \eta_{3}} \otimes \theta
$$

where we use the abbreviation,

$$
\begin{aligned}
* \zeta_{\eta_{1}, \eta_{2}, \eta_{3}} & =-\sigma^{2} \eta_{2} \zeta_{-\eta_{1},-\eta_{2},-\eta_{3}}^{*} \\
* \zeta & =-i \tau^{(121)} \sigma^{2} \zeta^{*}
\end{aligned}
$$

in $\tau$-matrix notation.

\section{B.1 The dilatino equation}

The dilatino equation is,

$$
0=i(P \cdot \Gamma) \mathcal{B}^{-1} \varepsilon^{*}-\frac{i}{24} \Gamma \cdot G \varepsilon
$$

Using the above form of the $\Gamma$-matrices, we obtain,

$$
0=\sum_{\eta_{1}, \eta_{2}, \eta_{3}} \chi^{\eta_{1}, \eta_{2}} \chi^{\eta_{3}} \otimes\left[i p_{a} \sigma^{a} \sigma^{2} \eta_{2} \zeta_{-\eta_{1}, \eta_{2},-\eta_{3}}^{*}+\frac{1}{4} i g_{\tilde{a}} \sigma^{\tilde{a}} \zeta_{\eta_{1}, \eta_{2}, \eta_{3}}+\frac{1}{4} h \zeta_{\eta_{1},-\eta_{2}, \eta_{3}}\right] \otimes \theta^{*}
$$

Since the $\chi^{\eta_{1}, \eta_{2}} \chi^{\eta_{3}}$ are linearly independent, each coefficient is required to vanish. The result may be recast economically using the $\tau$-matrix notation, and upon multiplication on the left by $-4 i \tau^{(131)}$, we have,

$$
0=4 p_{a} \sigma^{a} \sigma^{2} \zeta^{*}+g_{\tilde{a}} \tau^{(131)} \sigma^{\tilde{a}} \zeta+h \tau^{(121)} \zeta
$$

\section{B.2 The gravitino equation}

The gravitino equation is

$$
\begin{aligned}
0 & =d \varepsilon+\omega \varepsilon+\phi \varepsilon+g \mathcal{B}^{-1} \varepsilon^{*} \\
\omega & =\frac{1}{4} \omega_{A B} \Gamma^{A B} \\
\phi & =-\frac{i}{2} Q+\frac{i}{480}\left(\Gamma \cdot F_{(5)}\right) e_{A} \Gamma^{A} \\
g & =-\frac{1}{96} e_{A}\left(\Gamma^{A}(\Gamma \cdot G)+2(\Gamma \cdot G) \Gamma^{A}\right)
\end{aligned}
$$


The non-vanishing spin connection components are as follows,

$$
\begin{array}{ll}
\omega^{m}{ }_{n}=\hat{\omega}^{m}{ }_{n} & \omega^{m}{ }_{a}=e^{m} \frac{D_{a} f_{5}}{f_{5}} \\
\omega^{i}{ }_{j}=\hat{\omega}^{i}{ }_{j} & \omega^{i}{ }_{a}=e^{i} \frac{D_{a} f_{2}}{f_{2}} \\
\omega^{a}{ }_{b} & \omega^{9}{ }_{a}=e^{9} \frac{D_{a} f_{1}}{f_{1}}
\end{array}
$$

where $D_{a}=\rho^{-1} \partial_{a}$. The hats refers to the canonical connections on $A d S_{5}, S^{2}$ respectively. Projecting the spin-connection along the various directions we have the expression for $d \varepsilon+\omega \varepsilon$

$$
\begin{array}{ll}
\text { (m) } & \nabla_{m}^{\prime} \varepsilon+\frac{1}{2} \frac{D_{a} f_{5}}{f_{5}} \Gamma_{m} \Gamma^{a} \varepsilon \\
(i) & \nabla_{i}^{\prime} \varepsilon+\frac{1}{2} \frac{D_{a} f_{2}}{f_{2}} \Gamma_{i} \Gamma^{a} \varepsilon \\
(a) & \nabla_{a} \varepsilon \\
\text { (9) } & \nabla_{9}^{\prime} \varepsilon+\frac{1}{2} \frac{D_{a} f_{1}}{f_{1}} \Gamma_{9} \Gamma^{a} \varepsilon
\end{array}
$$

where the prime on the covariant derivative indicates that only the connection along $A d S_{5}$, $S^{2}$ respectively is included. Using the Killing spinor equations (3.1) we eliminate the primed covariant derivatives, and bring out overall factors of $\Gamma_{M}$, which yields

$$
\begin{array}{ll}
\text { (m) } & \Gamma_{m} \sum_{\eta_{1}, \eta_{2}, \eta_{3}} \chi^{\eta_{1}, \eta_{2}} \chi^{\eta_{3}} \otimes\left[\frac{i \eta_{1}}{2 f_{5}} \zeta_{\eta_{1}, \eta_{2}, \eta_{3}}+\frac{1}{2} \frac{D_{a} f_{5}}{f_{5}} \sigma^{a} \zeta_{\eta_{1},-\eta_{2}, \eta_{3}}\right] \otimes \theta^{*} \\
\text { (i) } & \Gamma_{i} \sum_{\eta_{1}, \eta_{2}, \eta_{3}} \chi^{\eta_{1}, \eta_{2}} \chi^{\eta_{3}} \otimes\left[\frac{i \eta_{2}}{2 f_{2}} \zeta_{\eta_{1}, \eta_{2}, \eta_{3}}+\frac{1}{2} \frac{D_{a} f_{2}}{f_{2}} \sigma^{a} \zeta_{\eta_{1},-\eta_{2}, \eta_{3}}\right] \otimes \theta^{*} \\
\text { (a) } & \nabla_{a} \varepsilon \\
\text { (9) } & \Gamma_{9} \sum_{\eta_{1}, \eta_{2}, \eta_{3}} \chi^{\eta_{1}, \eta_{2}} \chi^{\eta_{3}} \otimes\left[\frac{i \eta_{3}}{2 f_{1}} \sigma^{3} \zeta_{\eta_{1},-\eta_{2}, \eta_{3}}+\frac{1}{2} \frac{D_{a} f_{1}}{f_{1}} \sigma^{a} \zeta_{\eta_{1},-\eta_{2}, \eta_{3}}\right] \otimes \theta^{*}
\end{array}
$$

All terms in the gravitino equations (m), (i), and (9), will contain $\Gamma_{M} \chi^{\eta_{1}, \eta_{2}} \chi_{\eta_{3}}$, and we must require the coefficients to vanish independently. The coefficients of $\Gamma_{M} \chi^{\eta_{1}, \eta_{2}} \chi^{\eta_{3}}$ can be expressed in the $\tau$-matrix notation as

$$
\begin{array}{ll}
\text { (m) } & \frac{i}{2 f_{5}} \tau^{(300)} \zeta+\frac{1}{2} \frac{D_{a} f_{5}}{f_{5}} \tau^{(010)} \sigma^{a} \zeta \\
\text { (i) } & \frac{i}{2 f_{2}} \tau^{(030)} \zeta+\frac{1}{2} \frac{D_{a} f_{2}}{f_{2}} \tau^{(010)} \sigma^{a} \zeta \\
\text { (9) } & \frac{i}{2 f_{1}} \tau^{(013)} \sigma^{3} \zeta+\frac{1}{2} \frac{D_{a} f_{1}}{f_{1}} \tau^{(010)} \sigma^{a} \zeta
\end{array}
$$

The $F_{(5)}$ part in the calculation of $\phi$ is as follows,

$$
\frac{i}{480}\left(\Gamma \cdot F_{(5)}\right) \Gamma^{A} e_{A} \varepsilon=\frac{i}{2} f \Gamma^{01234} \Gamma^{A} e_{A} \varepsilon
$$


Projecting along the various directions, we have

$$
\begin{array}{ll}
\text { (m) } & \Gamma_{m} \sum_{\eta_{1}, \eta_{2}, \eta_{3}} \chi^{\eta_{1}, \eta_{2}} \chi^{\eta_{3}} \otimes-\frac{i}{2} f \zeta_{\eta_{1}, \eta_{2}, \eta_{3}} \otimes \theta^{*} \\
(i) & \Gamma_{i} \sum_{\eta_{1}, \eta_{2}, \eta_{3}} \chi^{\eta_{1}, \eta_{2}} \chi^{\eta_{3}} \otimes \frac{i}{2} f \zeta_{\eta_{1}, \eta_{2}, \eta_{3}} \otimes \theta^{*} \\
(a) & \sum_{\eta_{1}, \eta_{2}, \eta_{3}} \chi^{\eta_{1}, \eta_{2}} \chi^{\eta_{3}} \otimes\left[-\frac{i q_{a}}{2} \zeta_{\eta_{1}, \eta_{2}, \eta_{3}}+\frac{i}{2} f \sigma^{a} \zeta_{\eta_{1},-\eta_{2}, \eta_{3}}\right] \otimes \theta \\
(9) & \Gamma_{9} \sum_{\eta_{1}, \eta_{2}, \eta_{3}} \chi^{\eta_{1}, \eta_{2}} \chi^{\eta_{3}} \otimes\left[\frac{i}{2} f \zeta_{\eta_{1}, \eta_{2}, \eta_{3}}\right] \otimes \theta^{*}
\end{array}
$$

Using the $\tau$-matrix notation, we write the coefficients of $\Gamma_{M} \chi^{\eta_{1}, \eta_{2}} \chi^{\eta_{3}}$ or $\chi^{\eta_{1}, \eta_{2}} \chi^{\eta_{3}}$ in the form

$$
\begin{array}{ll}
\text { (m) } & -\frac{i}{2} f \zeta \\
(i) & \frac{i}{2} f \zeta \\
\text { (a) } & -\frac{i q_{a}}{2} \zeta+\frac{i f}{2} \tau^{(010)} \sigma^{a} \zeta \\
\text { (9) } & \frac{i}{2} f \zeta
\end{array}
$$

The relevant expression for the calculation of $g$ is as follows,

$$
g \mathcal{B}^{-1} \epsilon^{*}=-\frac{3 !}{96} e_{A}\left(i g_{\tilde{a}}\left(\Gamma^{A} \Gamma^{56 \tilde{a}}+2 \Gamma^{56 \tilde{a}} \Gamma^{A}\right)+h\left(\Gamma^{A} \Gamma^{789}+2 \Gamma^{789} \Gamma^{A}\right)\right) \mathcal{B}^{-1} \epsilon^{*}
$$

A few useful equations are as follows,

$$
\begin{aligned}
\Gamma^{\tilde{a}} \Gamma^{56 \tilde{b}}+2 \Gamma^{56 \tilde{b}} \Gamma^{\tilde{a}} & =\Gamma^{56}\left(3 \delta^{\tilde{\tilde{b}} \tilde{b}}-\Gamma^{\tilde{a} \tilde{b}}\right)=i \gamma_{(2)}\left(3 \delta^{\tilde{a} \tilde{b}}-\sigma^{\tilde{a} \tilde{b}}\right) \\
\Gamma^{\tilde{a}} \Gamma^{789}+2 \Gamma^{789} \Gamma^{\tilde{a}} & =3 \Gamma^{\tilde{a}} \Gamma^{789}
\end{aligned}
$$

Projecting along the various directions we obtain

$$
\begin{aligned}
& \text { (m) } \Gamma_{m} \sum_{\eta_{1}, \eta_{2}, \eta_{3}} \chi^{\eta_{1}, \eta_{2}} \chi^{\eta_{3}} \otimes \frac{1}{16}\left(-g_{\tilde{a}} \sigma^{\tilde{a}} * \zeta_{\eta_{1}, \eta_{2}, \eta_{3}}+i h * \zeta_{\eta_{1},-\eta_{2}, \eta_{3}}\right) \otimes \theta^{*} \\
& (i) \quad \Gamma_{i} \sum_{\eta_{1}, \eta_{2}, \eta_{3}} \chi^{\eta_{1}, \eta_{2}} \chi^{\eta_{3}} \otimes \frac{1}{16}\left(3 g_{\tilde{a}} \sigma^{\tilde{a}} * \zeta_{\eta_{1}, \eta_{2}, \eta_{3}}+i h * \zeta_{\eta_{1},-\eta_{2}, \eta_{3}}\right) \otimes \theta^{*} \\
& \left(\text { a) } \sum_{\eta_{1}, \eta_{2}, \eta_{3}} \chi^{\eta_{1}, \eta_{2}} \chi^{\eta_{3}} \otimes \frac{1}{16}\left(\left(3 g^{a}-g_{\tilde{b}} \sigma^{a \tilde{b}}\right) * \zeta_{\eta_{1},-\eta_{2}, \eta_{3}}-3 i h \sigma^{a} * \zeta_{\eta_{1}, \eta_{2}, \eta_{3}}\right) \otimes \theta\right. \\
& \text { (9) } \Gamma_{9} \sum_{\eta_{1}, \eta_{2}, \eta_{3}} \chi^{\eta_{1}, \eta_{2}} \chi^{\eta_{3}} \otimes \frac{1}{16}\left(\left(3 g^{9} \sigma^{9}-g_{a} \sigma^{a}\right) * \zeta_{\eta_{1}, \eta_{2}, \eta_{3}}-3 i h * \zeta_{\eta_{1},-\eta_{2}, \eta_{3}}\right) \otimes \theta^{*}
\end{aligned}
$$


Using the $\tau$-matrix notation, we write the coefficient of $\Gamma_{M} \chi^{\eta_{1}, \eta_{2}} \chi^{\eta_{3}}$ or $\chi^{\eta_{1}, \eta_{2}} \chi^{\eta_{3}}$ in the form

$$
\begin{aligned}
& \text { (m) } \frac{1}{16}\left(-g_{\tilde{a}} \sigma^{\tilde{a}} * \zeta+i h \tau^{(010)} * \zeta\right) \\
& \text { (i) } \frac{1}{16}\left(3 g_{\tilde{a}} \sigma^{\tilde{a}} * \zeta+i h \tau^{(010)} * \zeta\right) \\
& \text { (a) } \frac{1}{16}\left(3 g_{a} \tau^{(010)} * \zeta-g_{\tilde{b}} \tau^{(010)} \sigma_{a}^{\tilde{b}} * \zeta-3 i h \sigma_{a} * \zeta\right) \\
& \text { (9) } \frac{1}{16}\left(3 g^{9} \sigma^{3} * \zeta-g_{a} \sigma^{a} * \zeta-3 i h \tau^{(010)} * \zeta\right)
\end{aligned}
$$

\section{B.2.1 Assembling the complete gravitino BPS equation}

Now we combine the three equations (B.12), (B.15), and (B.19) to obtain the reduced gravitino equations. We again argue that the $\Gamma_{M} \chi^{\eta_{1}, \eta_{2}}$ or $\chi^{\eta_{1}, \eta_{2}}$ are linearly independent which leads to the equations (3.14).

For equations (m), (i) and (9), we have dropped an overall factor of $\Gamma_{M}$. In equation $(a), \hat{\omega}_{a}=\left(\hat{\omega}_{78}\right)_{a}$ is the spin connection along $\Sigma$, and we have used the connection formula (B.9) for the covariant derivative and the fact $\gamma^{78}=i \sigma^{3}$.

\section{Reducing the BPS equations: $A d S_{5} \times S^{3} \times \Sigma$ case}

In this appendix, we shall present the detailed calculations required for the reduction of the BPS equations in $A d S_{5} \times S^{3} \times \Sigma$ case. The corresponding $\Gamma$-matrices, in the basis given in appendix A.2, are given by,

$$
\begin{aligned}
\Gamma^{a} & =I_{4} \otimes I_{2} \otimes \gamma^{a} \otimes \sigma^{2} \\
\Gamma^{567} & =i I_{4} \otimes I_{2} \otimes I_{2} \otimes \sigma^{1} \\
\Gamma^{01234} & =i I_{4} \otimes I_{2} \otimes I_{2} \otimes \sigma^{3}
\end{aligned}
$$

We shall also need the decompositions of $\mathcal{B}^{-1} \varepsilon^{*}$,

$$
\mathcal{B}^{-1} \varepsilon^{*}=\sum_{\eta_{1}, \eta_{2}} \chi^{\eta_{1}, \eta_{2}} \otimes\left(-i \sigma^{1} \tau^{(10)} \zeta_{\eta_{1}, \eta_{2}}^{*} \otimes u_{+}+i \sigma^{2} \tau^{(10)} \zeta_{\eta_{1}, \eta_{2}}^{*} \otimes u_{-}\right)
$$

\section{C.1 The dilatino equation}

The dilatino equation becomes,

$$
\begin{aligned}
0= & i p_{a} \Gamma^{a} \sum_{\eta_{1}, \eta_{2}} \chi^{\eta_{1}, \eta_{2}} \otimes\left(-i \sigma^{1} \zeta_{-\eta_{1}, \eta_{2}}^{*} \otimes u_{+}+i \sigma^{2} \zeta_{-\eta_{1}, \eta_{2}}^{*} \otimes u_{-}\right) \\
& +\frac{1}{4} g \Gamma^{567} \sum_{\eta_{1}, \eta_{2}} \chi^{\eta_{1}, \eta_{2}} \otimes\left(\zeta_{\eta_{1}, \eta_{2}} \otimes u_{+}-i \sigma^{3} \zeta_{\eta_{1}, \eta_{2}} \otimes u_{-}\right)
\end{aligned}
$$

Using the above form of the matrices, we obtain,

$$
\begin{aligned}
& 0=i p_{a} \sigma^{a} \sigma^{2} \zeta_{-\eta_{1}, \eta_{2}}^{*}+\frac{1}{4} g \sigma^{3} \zeta_{\eta_{1}, \eta_{2}} \\
& 0=i p_{a} \sigma^{a} \sigma^{1} \zeta_{-\eta_{1}, \eta_{2}}^{*}+\frac{i}{4} g \zeta_{\eta_{1}, \eta_{2}}
\end{aligned}
$$


Multiplying the first equation by $\sigma^{3}$, and combined the result with the second equation, we find the reduced dilatino equations,

$$
\text { (d) } \quad 0=g \zeta_{\eta_{1}, \eta_{2}}=p_{a} \sigma^{a} \sigma^{1} \zeta_{-\eta_{1}, \eta_{2}}^{*}
$$

\section{C.2 The gravitino equation}

The gravitino equation is the same as equation (B.8). The non-vanishing spin connection components are $\omega_{b}^{a}$ and

$$
\begin{aligned}
\omega^{m}{ }_{n} & =\hat{\omega}^{m}{ }_{n} & \omega^{m}{ }_{a} & =e^{m} \frac{D_{a} f_{5}}{f_{5}} \\
\omega^{i}{ }_{j} & =\hat{\omega}^{i}{ }_{j} & \omega^{i}{ }_{a} & =e^{i} \frac{D_{a} f_{3}}{f_{3}}
\end{aligned}
$$

The hats refers to the canonical connections on $A d S_{5}, S^{3}$ respectively. Projecting the spin-connection along the various directions we have the expression for $d \varepsilon+\omega \varepsilon$

$$
\begin{array}{ll}
\text { (m) } & \nabla_{m}^{\prime} \varepsilon+\frac{1}{2} \frac{D_{a} f_{5}}{f_{5}} \Gamma_{m} \Gamma^{a} \varepsilon \\
(i) & \nabla_{i}^{\prime} \varepsilon+\frac{1}{2} \frac{D_{a} f_{3}}{f_{3}} \Gamma_{i} \Gamma^{a} \varepsilon \\
\text { (a) } & \nabla_{a} \varepsilon
\end{array}
$$

where the prime on the covariant derivative indicates that only the connection along $A d S_{5}$, $S^{3}$ respectively is included. Using the Killing spinor equations (8.5) we can eliminate the primed covariant derivatives, which yields

$$
\begin{array}{ll}
\text { (m) } & \frac{1}{2 f_{5}} \Gamma_{m} \sum_{\eta_{1}, \eta_{2}} \eta_{1} \chi^{\eta_{1}, \eta_{2}} \otimes\left(\zeta_{\eta_{1}, \eta_{2}} \otimes u_{+}+i \sigma^{3} \zeta_{\eta_{1}, \eta_{2}} \otimes u_{-}\right)+\frac{1}{2} \frac{D_{a} f_{5}}{f_{5}} \Gamma_{m} \Gamma^{a} \varepsilon \\
\text { (i) } & \frac{i}{2 f_{3}} \Gamma_{i} \sum_{\eta_{1}, \eta_{2}} \eta_{2} \chi^{\eta_{1}, \eta_{2}} \otimes\left(-i \sigma^{3} \zeta_{\eta_{1}, \eta_{2}} \otimes u_{+}+\zeta_{\eta_{1}, \eta_{2}} \otimes u_{-}\right)+\frac{1}{2} \frac{D_{a} f_{3}}{f_{3}} \Gamma_{i} \Gamma^{a} \varepsilon \\
\text { (a) } & \nabla_{a} \varepsilon
\end{array}
$$

combining the terms, we have

$$
\begin{array}{ll}
(m) \quad \Gamma_{m} \sum_{\eta_{1}, \eta_{2}} \chi^{\eta_{1}, \eta_{2}} \otimes\left[\left(\frac{\eta_{1}}{2 f_{5}} \zeta_{\eta_{1}, \eta_{2}}-\frac{1}{2} \frac{D_{a} f_{5}}{f_{5}} \sigma^{a} \sigma^{3} \zeta_{\eta_{1}, \eta_{2}}\right) \otimes u_{+}\right. \\
\left.+\left(\frac{i \eta_{1}}{2 f_{5}} \sigma^{3} \zeta_{\eta_{1}, \eta_{2}}+\frac{i}{2} \frac{D_{a} f_{5}}{f_{5}} \sigma^{a} \zeta_{\eta_{1}, \eta_{2}}\right) \otimes u_{-}\right] \\
\quad \Gamma_{i} \sum_{\eta_{1}, \eta_{2}} \chi^{\eta_{1}, \eta_{2}} \otimes\left[\left(\frac{\eta_{2}}{2 f_{3}} \sigma^{3} \zeta_{\eta_{1}, \eta_{2}}-\frac{1}{2} \frac{D_{a} f_{3}}{f_{3}} \sigma^{a} \sigma^{3} \zeta_{\eta_{1}, \eta_{2}}\right) \otimes u_{+}\right. \\
\left.+\left(\frac{i \eta_{2}}{2 f_{3}} \zeta_{\eta_{1}, \eta_{2}}+\frac{i}{2} \frac{D_{a} f_{3}}{f_{3}} \sigma^{a} \zeta_{\eta_{1}, \eta_{2}}\right) \otimes u_{-}\right] \\
\quad \nabla_{a} \varepsilon
\end{array}
$$

where we have pulled a factor of $\Gamma_{M}$ out front for equation (m), (i). It will turn out that all terms in the gravitino equation (m) (i) will contain $\Gamma_{M} \chi^{\eta_{1}, \eta_{2}}$, and we require the 
coefficients to vanish independently, just as we did for the dilatino equation. The coefficient of $\Gamma_{M} \chi^{\eta_{1}, \eta_{2}}, \chi^{\eta_{3}}$ can be expressed in the $\tau$-matrix notation as

$\begin{array}{ll}\text { (m) } & \left(\frac{1}{2 f_{5}} \tau^{30}-\frac{1}{2} \frac{D_{a} f_{5}}{f_{5}} \sigma^{a} \sigma^{3}\right) \zeta \otimes u_{+}+\left(\frac{i}{2 f_{5}} \tau^{30} \sigma^{3}+\frac{i}{2} \frac{D_{a} f_{5}}{f_{5}} \sigma^{a}\right) \zeta \otimes u_{-} \\ \text {(i) } & \left(\frac{1}{2 f_{3}} \tau^{03} \sigma^{3}-\frac{1}{2} \frac{D_{a} f_{3}}{f_{3}} \sigma^{a} \sigma^{3}\right) \zeta \otimes u_{+}+\left(\frac{i}{2 f_{3}} \tau^{03}+\frac{i}{2} \frac{D_{a} f_{3}}{f_{3}} \sigma^{a}\right) \zeta \otimes u_{-}\end{array}$

The $F_{(5)}$ part is

$$
\frac{i}{480}\left(\Gamma \cdot F_{(5)}\right) \Gamma^{A} e_{A} \varepsilon=\frac{i}{2} f \Gamma^{01234} \Gamma^{A} e_{A} \varepsilon
$$

Projecting along the various directions, we have

$$
\begin{aligned}
& \text { (m) } \Gamma_{m} \sum_{\eta_{1}, \eta_{2}} \chi^{\eta_{1}, \eta_{2}} \otimes\left(-\frac{f}{2} \zeta_{\eta_{1}, \eta_{2}} \otimes u_{+}-\frac{i f}{2} \sigma^{3} \zeta_{\eta_{1}, \eta_{2}} \otimes u_{-}\right) \\
& \text {(i) } \Gamma_{i} \sum_{\eta_{1}, \eta_{2}} \chi^{\eta_{1}, \eta_{2}} \otimes\left(\frac{f}{2} \zeta_{\eta_{1}, \eta_{2}} \otimes u_{+}+\frac{i f}{2} \sigma^{3} \zeta_{\eta_{1}, \eta_{2}} \otimes u_{-}\right) \\
& \text {(a) } \sum_{\eta_{1}, \eta_{2}} \chi^{\eta_{1}, \eta_{2}} \otimes\left(\left(\frac{f}{2} \sigma_{a} \sigma^{3}-\frac{i}{2} q_{a}\right) \zeta_{\eta_{1}, \eta_{2}} \otimes u_{+}+\left(\frac{i f}{2} \sigma_{a}-\frac{1}{2} q_{a} \sigma^{3}\right) \zeta_{\eta_{1}, \eta_{2}} \otimes u_{-}\right)
\end{aligned}
$$

\section{C.2.1 Assembling the complete gravitino BPS equation}

Now we combine the above equations to obtain the reduced gravitino equations. We again argue that the $\Gamma_{M} \chi^{\eta_{1}, \eta_{2}}$ or $\chi^{\eta_{1}, \eta_{2}}$ are linearly independent which leads to the equations

$$
\begin{aligned}
\text { (m) } 0 & =\left(\frac{1}{2 f_{5}} \tau^{(30)}-\frac{1}{2} \frac{D_{a} f_{5}}{f_{5}} \sigma^{a} \sigma^{3}-\frac{f}{2}\right) \zeta \otimes u_{+}+\left(\frac{i}{2 f_{5}} \tau^{(30)} \sigma^{3}+\frac{i}{2} \frac{D_{a} f_{5}}{f_{5}} \sigma^{a}-\frac{i f}{2} \sigma^{3}\right) \zeta \otimes u_{-} \\
\text {(i) } 0 & =\left(\frac{1}{2 f_{3}} \tau^{(03)} \sigma^{3}-\frac{1}{2} \frac{D_{a} f_{3}}{f_{3}} \sigma^{a} \sigma^{3}+\frac{f}{2}\right) \zeta \otimes u_{+}+\left(\frac{i}{2 f_{3}} \tau^{(03)}+\frac{i}{2} \frac{D_{a} f_{3}}{f_{3}} \sigma^{a}+\frac{i f}{2} \sigma^{3}\right) \zeta \otimes u_{-} \\
\text {(a) } 0 & =\left(D_{a}+\frac{i}{2} \hat{\omega}_{a} \sigma^{3}+\frac{f}{2} \sigma_{a} \sigma^{3}-\frac{i}{2} q_{a}\right) \zeta \otimes u_{+}-i \sigma^{3}\left(D_{a}+\frac{i}{2} \hat{\omega}_{a} \sigma^{3}+\frac{f}{2} \sigma_{a} \sigma^{3}-\frac{i}{2} q_{a}\right) \zeta \otimes u_{-}
\end{aligned}
$$

Separating $u_{+}$and $u_{-}$terms reproduces the equations of (8.11).

\section{Calculation of metric factors for $A d S_{5} \times S^{2} \times S^{1} \times \Sigma$}

We shall use combinations of the differential equations $( \pm)$ and of the algebraic gravitino BPS equations of (3.27) to bring out the corresponding relations. To this end, we compute,

$$
\begin{aligned}
D_{z}\left(\psi^{\dagger} \tau^{(\alpha \beta)} \psi\right)= & -f \psi^{\dagger} \tau^{(\alpha \beta)} \tau^{(12)} \xi^{*}+\frac{i}{4} g_{\bar{z}}^{*} \xi^{\dagger} \tau^{(\alpha \beta)} \psi \\
& -\frac{i}{8}\left(g_{z} \psi^{\dagger} \tau^{(\alpha \beta)} \xi+g_{9} \psi^{\dagger} \tau^{(\alpha \beta)} \tau^{(13)} \psi^{*}+3 h \psi^{\dagger} \tau^{(\alpha \beta)} \tau^{(12)} \psi^{*}\right) \\
D_{z}\left(\xi^{\dagger} \tau^{(\alpha \beta)} \xi\right)= & +f \psi^{\dagger} \tau^{(12)}\left(\tau^{(\alpha \beta)}\right)^{t} \xi^{*}+\frac{i}{4} g_{z} \psi^{\dagger} \tau^{(\alpha \beta)} \xi \\
& +\frac{i}{8}\left(-g_{\bar{z}}^{*} \xi^{\dagger} \tau^{(\alpha \beta)} \psi+g_{9}^{*} \xi^{\dagger} \tau^{(\alpha \beta)} \tau^{(13)} \xi^{*}-3 h^{*} \xi^{\dagger} \tau^{(\alpha \beta)} \tau^{(12)} \xi^{*}\right)
\end{aligned}
$$


In the subsequent subsections, we shall combine these results with corresponding combinations from the algebraic gravitino BPS equations, and seek relations of the following type,

$$
D_{z}\left(r_{1} \psi^{\dagger} \tau^{(\alpha \beta)} \psi+r_{2} \xi^{\dagger} \tau^{(\alpha \beta)} \xi\right)+\frac{D_{z} f_{i}}{f_{i}}\left(r_{3} \psi^{\dagger} \tau^{(\alpha \beta)} \psi+r_{4} \xi^{\dagger} \tau^{(\alpha \beta)} \xi\right)=0
$$

where $i=1,2,5$ and the coefficients $r_{1}, r_{2}, r_{3}, r_{4}$ may depend on $i$, and $\alpha, \beta$, but not on $\Sigma$. We shall seek such relations for generic fields $f_{1}, f_{2}, f_{5}, f, g_{z}, g_{\bar{z}}, h$, and $g_{9}$, though we shall find that when special relations hold (such as $g_{9}=0$ ), additional relations of type (D.2) exist.

\section{D.1 Relations involving $f_{5}$}

We start with the metric factor $f_{5}$, provided by the $(m)$-equation. Left-multiplying equation $\left(m_{1}\right)$ by $\psi^{\dagger}$, and left-multiplying the complex conjugate of equation $\left(m_{2}\right)$ by $\xi^{\dagger}$, we find,

$$
\begin{aligned}
\frac{D_{z} f_{5}}{f_{5}}\left(\psi^{\dagger} \tau^{(\alpha \beta)} \psi\right)= & -\frac{i}{f_{5}} \psi^{\dagger} \tau^{(\alpha \beta)} \tau^{(22)} \xi^{*}+f \psi^{\dagger} \tau^{(\alpha \beta)} \tau^{(12)} \xi^{*} \\
& +\frac{i}{8}\left(g_{z} \psi^{\dagger} \tau^{(\alpha \beta)} \xi-g_{9} \psi^{\dagger} \tau^{(\alpha \beta)} \tau^{(13)} \psi^{*}+h \psi^{\dagger} \tau^{(\alpha \beta)} \tau^{(12)} \psi^{*}\right) \\
\frac{D_{z} f_{5}}{f_{5}}\left(\xi^{\dagger} \tau^{(\alpha \beta)} \xi\right)= & +\frac{i}{f_{5}} \xi^{\dagger} \tau^{(\alpha \beta)} \tau^{(22)} \psi^{*}+f \xi^{\dagger} \tau^{(\alpha \beta)} \tau^{(12)} \psi^{*} \\
& +\frac{i}{8}\left(g_{\bar{z}}^{*} \xi^{\dagger} \tau^{(\alpha \beta)} \psi+g_{9}^{*} \xi^{\dagger} \tau^{(\alpha \beta)} \tau^{(13)} \xi^{*}+h^{*} \xi^{\dagger} \tau^{(\alpha \beta)} \tau^{(12)} \xi^{*}\right)
\end{aligned}
$$

For generic fields, term by term cancellation imposes the following requirements,

$$
\begin{aligned}
\left(f_{5}\right) & 0=-r_{3} \tau^{(\alpha \beta)} \tau^{(22)}+r_{4} \tau^{(22)}\left(\tau^{(\alpha \beta)}\right)^{t} \\
(f) & 0=\left(r_{3}-r_{1}\right) \tau^{(\alpha \beta)} \tau^{(12)}+\left(r_{2}-r_{4}\right) \tau^{(12)}\left(\tau^{(\alpha \beta)}\right)^{t} \\
\left(g_{\bar{z}}^{*}\right) & 0=2 r_{1}-r_{2}+r_{4} \\
\left(g_{z}\right) & 0=2 r_{2}-r_{1}+r_{3} \\
\left(g_{9}\right) & 0=\left(r_{1}+r_{3}\right) g_{9} \psi^{\dagger} \tau^{(\alpha \beta)} \tau^{(13)} \psi^{*}=\left(r_{2}+r_{4}\right) g_{9}^{*} \xi^{\dagger} \tau^{(\alpha \beta)} \tau^{(13)} \xi^{*} \\
(h) & 0=\left(3 r_{1}-r_{3}\right) h \psi^{\dagger} \tau^{(\alpha \beta)} \tau^{(12)} \psi^{*}=\left(3 r_{2}-r_{4}\right) h^{*} \xi^{\dagger} \tau^{(\alpha \beta)} \tau^{(12)} \xi^{*}
\end{aligned}
$$$$
\left(g_{z}\right) \quad 0=2 r_{2}-r_{1}+r_{3}
$$

We shall now systematically analyze and solve the above conditions.

If $r_{3}=0$, then equation $\left(f_{5}\right)$ implies $r_{4}=0$, and $\left(g_{z}\right)$ and $\left(g_{\bar{z}}^{*}\right)$ imply $r_{1}=r_{2}=0$, which yields the trivial solution. Thus, we have $r_{3} \neq 0$, and by an overall rescaling, we choose $r_{3}=1$. Equation $\left(f_{5}\right)$ then implies that we must have $\left|r_{4}\right|=1$.

Equations $\left(g_{\bar{z}}^{*}\right)$ and $\left(g_{z}\right)$ then reduce to $r_{1}=2 r_{2}+1$, and $r_{4}=-3 r_{2}-2$, supplemented with the condition $\left|r_{4}\right|=\left|3 r_{2}+2\right|=1$. These relations have two distinct solutions, according to whether $r_{4}= \pm 1$. Satisfying the $(f)$ and $\left(f_{5}\right)$ equations then gives two sets of solutions,

$$
\begin{array}{ll}
\left(r_{1}, r_{2}, r_{3}, r_{4}\right)=(-1,-1,1,1) & \tau^{(\alpha \beta)} \in\left\{\tau^{(00)}, \tau^{(31)}, \tau^{(32)}, \tau^{(33)}\right\} \\
\left(r_{1}, r_{2}, r_{3}, r_{4}\right)=(1 / 3,-1 / 3,1,-1) & \tau^{(\alpha \beta)}=\left\{\tau^{(01)}, \tau^{(02)}, \tau^{(03)}, \tau^{(30)}\right\}
\end{array}
$$


Equations $(h)$ automatically holds for both solutions, while equations $\left(g_{9}\right)$ hold automatically for the first solution, and with $\tau^{(\alpha \beta)} \in\left\{\tau^{(01)}, \tau^{(30)}\right\}$ for the second solution. Thus, the first solution is generic, and the second solution is generic with $\tau^{(\alpha \beta)} \in\left\{\tau^{(01)}, \tau^{(30)}\right\}$.

\section{D.2 Relations involving $f_{2}$}

The analysis of the bilinear relations involving $f_{2}$ is analogous. We start from the relations,

$$
\begin{aligned}
\frac{D_{z} f_{2}}{f_{2}}\left(\psi^{\dagger} \tau^{(\alpha \beta)} \psi\right)= & -\frac{i}{f_{2}} \psi^{\dagger} \tau^{(\alpha \beta)} \tau^{(11)} \xi^{*}-f \psi^{\dagger} \tau^{(\alpha \beta)} \tau^{(12)} \xi^{*} \\
& +\frac{i}{8}\left(-3 g_{z} \psi^{\dagger} \tau^{(\alpha \beta)} \xi+3 g_{9} \psi^{\dagger} \tau^{(\alpha \beta)} \tau^{(13)} \psi^{*}+h \psi^{\dagger} \tau^{(\alpha \beta)} \tau^{(12)} \psi^{*}\right) \\
\frac{D_{z} f_{2}}{f_{2}}\left(\xi^{\dagger} \tau^{(\alpha \beta)} \xi\right)= & -\frac{i}{f_{2}} \xi^{\dagger} \tau^{(\alpha \beta)} \tau^{(11)} \psi^{*}-f \xi^{\dagger} \tau^{(\alpha \beta)} \tau^{(12)} \psi^{*} \\
& -\frac{i}{8}\left(3 g_{\bar{z}}^{*} \xi^{\dagger} \tau^{(\alpha \beta)} \psi+3 g_{9}^{*} \xi^{\dagger} \tau^{(\alpha \beta)} \tau^{(13)} \xi^{*}-h^{*} \xi^{\dagger} \tau^{(\alpha \beta)} \tau^{(12)} \xi^{*}\right)
\end{aligned}
$$

For generic fields, term by term cancellation imposes the following requirements,

$$
\begin{aligned}
\left(f_{2}\right) & 0=r_{3} \tau^{(\alpha \beta)} \tau^{(11)}+r_{4} \tau^{(11)}\left(\tau^{(\alpha \beta)}\right)^{t} \\
(f) & 0=-\left(r_{1}+r_{3}\right) \tau^{(\alpha \beta)} \tau^{(12)}+\left(r_{2}+r_{4}\right) \tau^{(12)}\left(\tau^{(\alpha \beta)}\right)^{t} \\
\left(g_{z}^{*}\right) & 0=2 r_{1}-r_{2}-3 r_{4} \\
\left(g_{z}\right) & 0=2 r_{2}-r_{1}-3 r_{3} \\
\left(g_{9}\right) & 0=\left(3 r_{3}-r_{1}\right) g_{9} \psi^{\dagger} \tau^{(\alpha \beta)} \tau^{(13)} \psi^{*}=\left(3 r_{4}-r_{2}\right) g_{9}^{*} \xi^{\dagger} \tau^{(\alpha \beta)} \tau^{(13)} \xi^{*} \\
(h) & 0=\left(3 r_{1}-r_{3}\right) h \psi^{\dagger} \tau^{(\alpha \beta)} \tau^{(12)} \psi^{*}=\left(3 r_{2}-r_{4}\right) h^{*} \xi^{\dagger} \tau^{(\alpha \beta)} \tau^{(12)} \xi^{*}
\end{aligned}
$$

We may again set $r_{3}=1$, so that $\left|r_{4}\right|=1$ without loss of generality. Equations $\left(g_{\bar{z}}^{*}\right)$ and $\left(g_{z}\right)$ reduce to $r_{1}=2 r_{2}-3$, and $r_{4}=r_{2}-2$, supplemented with the condition $\left|r_{4}\right|=\left|r_{2}-2\right|=1$. Equations $(f)$ and $\left(f_{2}\right)$ leave two distinct solutions, given by

$$
\begin{array}{cc}
\left(r_{1}, r_{2}, r_{3}, r_{4}\right)=(-1,1,1,-1) \quad & \tau^{(\alpha \beta)} \in\left\{\tau^{(00)}, \tau^{(01)}, \tau^{(02)}, \tau^{(10)}, \tau^{(11)},\right. \\
& \left.\tau^{(12)}, \tau^{(20)}, \tau^{(21)}, \tau^{(22)}, \tau^{(33)}\right\} \\
\left(r_{1}, r_{2}, r_{3}, r_{4}\right)=(3,3,1,1) & \tau^{(\alpha \beta)} \in\left\{\tau^{(31)}, \tau^{(32)}\right\}
\end{array}
$$

Equations $(h),\left(g_{9}\right)$ automatically hold for the second solution. They hold for the first solution with $\tau^{(\alpha \beta)}=\tau^{(33)}$, which is generic; with $\tau^{(00)}, \tau^{(10)}, \tau^{(20)}$ when $g_{9}=0$, with $\tau^{(01)}, \tau^{(11)}, \tau^{(21)}$ when $h=0$, and with $\tau^{(02)}, \tau^{(12)}, \tau^{(22)}$ when $g_{9}=h=0$.

\section{D.3 Relations involving $f_{1}$}

The analysis of the bilinear relations involving $f_{1}$ is analogous. We start from the relations,

$$
\begin{aligned}
\frac{D_{z} f_{1}}{f_{1}}\left(\psi^{\dagger} \tau^{(\alpha \beta)} \psi\right)= & \frac{\nu}{f_{1}} \psi^{\dagger} \tau^{(\alpha \beta)} \tau^{(23)} \xi^{*}-f \psi^{\dagger} \tau^{(\alpha \beta)} \tau^{(12)} \xi^{*} \\
& +\frac{i}{8}\left(g_{z} \psi^{\dagger} \tau^{(\alpha \beta)} \xi+3 g_{9} \psi^{\dagger} \tau^{(\alpha \beta)} \tau^{(13)} \psi^{*}-3 h \psi^{\dagger} \tau^{(\alpha \beta)} \tau^{(12)} \psi^{*}\right) \\
\frac{D_{z} f_{1}}{f_{1}}\left(\xi^{\dagger} \tau^{(\alpha \beta)} \xi\right)= & -\frac{\nu}{f_{1}} \xi^{\dagger} \tau^{(\alpha \beta)} \tau^{(23)} \psi^{*}-f \xi^{\dagger} \tau^{(\alpha \beta)} \tau^{(12)} \psi^{*} \\
& +\frac{i}{8}\left(g_{\bar{z}}^{*} \xi^{\dagger} \tau^{(\alpha \beta)} \psi-3 g_{9}^{*} \xi^{\dagger} \tau^{(\alpha \beta)} \tau^{(13)} \xi^{*}-3 h^{*} \xi^{\dagger} \tau^{(\alpha \beta)} \tau^{(12)} \xi^{*}\right)
\end{aligned}
$$


For generic fields, term by term cancellation imposes the following requirements,

$$
\begin{aligned}
\left(f_{1}\right) & 0=r_{3} \tau^{(\alpha \beta)} \tau^{(23)}+r_{4} \tau^{(23)}\left(\tau^{(\alpha \beta)}\right)^{t} \\
(f) & 0=-\left(r_{1}+r_{3}\right) \tau^{(\alpha \beta)} \tau^{(12)}+\left(r_{2}+r_{4}\right) \tau^{(12)}\left(\tau^{(\alpha \beta)}\right)^{t} \\
\left(g_{z}^{*}\right) & 0=2 r_{1}-r_{2}+r_{4} \\
\left(g_{z}\right) & 0=2 r_{2}-r_{1}+r_{3} \\
\left(g_{9}\right) & 0=\left(3 r_{3}-r_{1}\right) g_{9} \psi^{\dagger} \tau^{(\alpha \beta)} \tau^{(13)} \psi^{*}=\left(3 r_{4}-r_{2}\right) g_{9}^{*} \xi^{\dagger} \tau^{(\alpha \beta)} \tau^{(13)} \xi^{*} \\
(h) & 0=\left(r_{1}+r_{3}\right) h \psi^{\dagger} \tau^{(\alpha \beta)} \tau^{(12)} \psi^{*}=\left(r_{2}+r_{4}\right) h^{*} \xi^{\dagger} \tau^{(\alpha \beta)} \tau^{(12)} \xi^{*}
\end{aligned}
$$

We may again set $r_{3}=1$, so that $\left|r_{4}\right|=1$ without loss of generality. Equations $\left(g_{\bar{z}}^{*}\right)$ and $\left(g_{z}\right)$ reduce to $r_{1}=2 r_{2}+1$, and $r_{4}=-3 r_{2}-2$, supplemented with the condition $\left|r_{4}\right|=\left|3 r_{2}+2\right|=1$. Equations $(f)$ and $\left(f_{1}^{-1}\right)$ leave two distinct solutions, given by

$$
\begin{aligned}
\left(r_{1}, r_{2}, r_{3}, r_{4}\right)=(-1,-1,1,1) & \tau^{(\alpha \beta)} \in\left\{\tau^{(01)}, \tau^{(10)}, \tau^{(12)}, \tau^{(13)}, \tau^{(20)},\right. \\
& \left.\tau^{(22)}, \tau^{(23)}, \tau^{(30)}, \tau^{(32)}, \tau^{(33)}\right\} \\
\left(r_{1}, r_{2}, r_{3}, r_{4}\right)=(1 / 3,-1 / 3,1,-1) & \tau^{(\alpha \beta)} \in\left\{\tau^{(02)}, \tau^{(03)}, \tau^{(11)}, \tau^{(21)}\right\}
\end{aligned}
$$

Equations $(h)$ automatically hold for the first solution, while $\left(g_{9}\right)$ automatically holds only with $\tau^{(\alpha \beta)} \in\left\{\tau^{(01)}, \tau^{(30)}, \tau^{(32)}, \tau^{(33)}\right\}$, which is the generic solution. It holds with $\tau^{(10)}, \tau^{(12)}, \tau^{(13)}, \tau^{(20)}, \tau^{(22)}, \tau^{(23)}$ when $g_{9}=0$. For the second solution, the equations hold with $\tau^{(11)}, \tau^{(21)}$ when $h=0$, and with $\tau^{(02)}, \tau^{(03)}$ when $g_{9}=h=0$.

\section{E Vanishing Hermitian forms for $A d S_{5} \times S^{2} \times S^{1} \times \Sigma$}

In this appendix, we derive three sets of vanishing Hermitian forms.

\section{E.1 First set of Hermitan relations}

The first set of Hermitian relations is obtained by considering the combination of the BPS equations of $(3.27)$, given by $2(m)+(i)+(9)$. All $f, g_{z}, g_{\bar{z}}, h, h^{*}$ terms cancel in these combinations, and the resulting equations are,

$$
\begin{aligned}
D_{z} \ln \left(f_{5}^{2} f_{2} f_{1}\right) \psi+\left(\frac{2 i}{f_{5}} \tau^{(22)}+\frac{i}{f_{2}} \tau^{(11)}-\frac{\nu}{f_{1}} \tau^{(23)}\right) \xi^{*}-\frac{i}{2} g_{9} \tau^{(13)} \psi^{*}=0 \\
D_{z} \ln \left(f_{5}^{2} f_{2} f_{1}\right) \xi+\left(-\frac{2 i}{f_{5}} \tau^{(22)}+\frac{i}{f_{2}} \tau^{(11)}+\frac{\nu}{f_{1}} \tau^{(23)}\right) \psi^{*}+\frac{i}{2} g_{9}^{*} \tau^{(13)} \xi^{*}=0
\end{aligned}
$$

Multiplying the first equation by $\xi^{t} \tau^{(\alpha \beta)}$, the second by $-\psi^{t} \tau^{(\alpha \beta) t}$, adding both to cancel the differential terms, and taking the transpose, we obtain,

$$
\begin{aligned}
0= & \xi^{\dagger}\left(\frac{2 i}{f_{5}} \tau^{(22)}+\frac{i}{f_{2}} \tau^{(11)}+\frac{\nu}{f_{1}} \tau^{(23)}\right) \tau^{(\alpha \beta)^{t}} \xi-\frac{i}{2} g_{9} \psi^{\dagger} \tau^{(13)} \tau^{(\alpha \beta)^{t}} \xi \\
& +\psi^{\dagger}\left(\frac{2 i}{f_{5}} \tau^{(22)}-\frac{i}{f_{2}} \tau^{(11)}+\frac{\nu}{f_{1}} \tau^{(23)}\right) \tau^{(\alpha \beta)} \psi-\frac{i}{2} g_{9}^{*} \xi^{\dagger} \tau^{(13)} \tau^{(\alpha \beta)} \psi
\end{aligned}
$$


Each term in $\xi^{\dagger} \tau \xi$ or $\psi^{\dagger} \tau \psi$, and each combination of terms involving $g_{9}$ and $g_{9}^{*}$, is either real or purely imaginary. Defining the sign factors \pm associated with each label $(\alpha \beta)$,

$$
\tau^{(\alpha \beta) *}= \pm \tau^{(13)} \tau^{(\alpha \beta)} \tau^{(13)}
$$

the corresponding term in $g_{9}$ and $g_{9}^{*}$ is real for the - sign and imaginary for the + sign. Separating out the corresponding real and imaginary parts yields valuable information. We summarize the resulting relations below, the first column corresponding to the $(\alpha \beta)$ assignment(s) from which the equation has been derived.

$$
\begin{aligned}
& H_{+}^{(23)}=0 \quad \frac{2}{f_{5}} H_{+}^{(22)}-\frac{1}{f_{2}} H_{-}^{(11)}-\frac{1}{2} H_{g+}^{(13)}=0 \\
& H_{g+}^{(12)}=0 \quad \frac{1}{f_{2}} H_{-}^{(10)}-\frac{\nu}{f_{1}} H_{+}^{(22)}=0 \\
& H_{+}^{(13)}=0 \quad \frac{2}{f_{5}} H_{-}^{(20)}-\frac{\nu}{f_{1}} H_{-}^{(21)}-\frac{i}{2} H_{g-}^{(11)}=0 \\
& H_{g+}^{(10)}=0 \quad \frac{2}{f_{5}} H_{+}^{(21)}+\frac{1}{f_{2}} H_{-}^{(12)}-\frac{\nu}{f_{1}} H_{+}^{(20)}=0 \\
& H_{+}^{(32)}=0 \quad \frac{1}{f_{2}} H_{-}^{(01)}+\frac{\nu}{f_{1}} H_{+}^{(33)}+\frac{1}{2} H_{g+}^{(03)}=0 \\
& H_{g+}^{(02)}=0 \quad \frac{2}{f_{5}} H_{+}^{(33)}+\frac{1}{f_{2}} H_{-}^{(00)}=0 \\
& H_{g-}^{(01)}=0 \quad \frac{2}{f_{5}} H_{-}^{(30)}+\frac{1}{f_{2}} H_{+}^{(03)}-\frac{\nu}{f_{1}} H_{-}^{(31)}=0 \\
& H_{-}^{(02)}=0 \quad \frac{2}{f_{5}} H_{+}^{(31)}-\frac{\nu}{f_{1}} H_{+}^{(30)}-\frac{1}{2} H_{g+}^{(00)}=0 \\
& H_{g-}^{(33)}=0 \quad \frac{1}{f_{2}} H_{+}^{(31)}+\frac{\nu}{f_{1}} H_{-}^{(03)}=0 \\
& H_{-}^{(02)}=0 \quad \frac{2}{f_{5}} H_{-}^{(03)}+\frac{1}{f_{2}} H_{+}^{(30)}-\frac{i}{2} H_{g-}^{(32)}=0 \\
& \frac{2}{f_{5}} H_{+}^{(00)}+\frac{1}{f_{2}} H_{-}^{(33)}-\frac{\nu}{f_{1}} H_{+}^{(01)}-\frac{1}{2} H_{g+}^{(31)}=0 \\
& H_{g-}^{(30)}=0 \quad \frac{2}{f_{5}} H_{-}^{(01)}-\frac{\nu}{f_{1}} H_{-}^{(00)}=0 \\
& H_{+}^{(13)}=0 \quad \frac{2}{f_{5}} H_{+}^{(12)}+\frac{1}{f_{2}} H_{-}^{(21)}+\frac{1}{2} H_{g+}^{(23)}=0 \\
& H_{g+}^{(22)}=0 \quad \frac{1}{f_{2}} H_{-}^{(20)}+\frac{\nu}{f_{1}} H_{+}^{(12)}=0 \\
& H_{+}^{(23)}=0 \quad \frac{2}{f_{5}} H_{-}^{(10)}-\frac{\nu}{f_{1}} H_{-}^{(11)}+\frac{i}{2} H_{g-}^{(21)}=0 \\
& H_{g+}^{(20)}=0 \quad \frac{2}{f_{5}} H_{+}^{(11)}-\frac{1}{f_{2}} H_{-}^{(22)}-\frac{\nu}{f_{1}} H_{+}^{(10)}=0
\end{aligned}
$$

\section{E.2 Second set of Hermitian relations}

The second set of Hermitian equalities is derived from eliminating $D_{z} f_{5}, D_{z} f_{2}, D_{z} f_{1}, g_{z}$, and $g_{\bar{z}}^{*}$ terms in each set of equations $(\mathrm{m})$, (i), or (9) respectively. Because the (9) equation 
can be obtained from a linear superpostion of $(\mathrm{m})$ and (i) equations, and those from the first set, we only need to calculate the $(\mathrm{m})$ and (i) equations.

\section{E.2.1 The $f_{5}$-equation}

For the $f_{5}$ equation, we multiply the first by $\xi^{t} \tau^{(\alpha \beta)}$, and the second by $\psi^{t} \tau^{(\alpha \beta)}$. The $g_{z}, g_{\bar{z}}$ terms will disappear if $\tau^{(\alpha \beta)}$ is anti-symmetric, $\tau^{(\alpha \beta) t}=-\tau^{(\alpha \beta)}$. Adding both equations to eliminate the $D_{z} f_{5}$-term, we obtain,

$$
\begin{aligned}
0= & \xi^{t} \tau^{(\alpha \beta)}\left(\frac{i}{f_{5}} \tau^{(22)} \xi^{*}-f \tau^{(12)} \xi^{*}+\frac{i}{8} g_{9} \tau^{(13)} \psi^{*}-\frac{i}{8} h \tau^{(12)} \psi^{*}\right) \\
& +\psi^{t} \tau^{(\alpha \beta)}\left(-\frac{i}{f_{5}} \tau^{(22)} \psi^{*}-f \tau^{(12)} \psi^{*}-\frac{i}{8} g_{9}^{*} \tau^{(13)} \xi^{*}-\frac{i}{8} h^{*} \tau^{(12)} \xi^{*}\right)
\end{aligned}
$$

Separating the real and imaginary parts for all antisymmetric $\tau^{(\alpha \beta)}$, we obtain,

$$
\begin{array}{ll}
H_{+}^{(10)}=0 & \frac{i}{f_{5}} H_{-}^{(20)}-\frac{1}{8} H_{g-}^{(11)}-\frac{i}{8} H_{h+}^{(10)}=0 \\
H_{h+}^{(32)}=0 & \frac{i}{f_{5}} H_{-}^{(02)}-i f H_{+}^{(32)}+\frac{1}{8} H_{g-}^{(33)}=0 \\
H_{g-}^{(01)}+i H_{h+}^{(00)}=0 & \frac{1}{f_{5}} H_{-}^{(30)}-f H_{+}^{(00)}=0 \\
H_{h+}^{(33)}=0 & \frac{1}{f_{5}} H_{-}^{(03)}-f H_{+}^{(33)}+\frac{i}{8} H_{g-}^{(32)}=0 \\
H_{+}^{(20)}=0 & \frac{1}{f_{5}} H_{-}^{(10)}-\frac{i}{8} H_{g-}^{(21)}+\frac{1}{8} H_{h+}^{(20)}=0 \\
H_{g-}^{(30)}+i H_{h+}^{(31)}=0 & \frac{1}{f_{5}} H_{-}^{(01)}-f H_{+}^{(31)}=0
\end{array}
$$

\section{E.2.2 The $f_{2}$-equation}

For the $f_{2}$ equations, with a similar procedure, we have the following result,

$$
\begin{aligned}
\xi^{t} \tau^{(\alpha \beta)}\left(\frac{i}{f_{2}} \tau^{(11)} \xi^{*}\right. & \left.+f \tau^{(12)} \xi^{*}-\frac{3 i}{8} g_{9} \tau^{(13)} \psi^{*}-\frac{i}{8} h \tau^{(12)} \psi^{*}\right) \\
& +\psi^{t} \tau^{(\alpha \beta)}\left(\frac{i}{f_{2}} \tau^{(11)} \psi^{*}+f \tau^{(12)} \psi^{*}+\frac{3 i}{8} g_{9}^{*} \tau^{(13)} \xi^{*}-\frac{i}{8} h^{*} \tau^{(12)} \xi^{*}\right)=0
\end{aligned}
$$

Separating into real and imaginary parts, we obtain,

$$
\begin{array}{ll}
\frac{1}{f_{2}} H_{+}^{(13)}+f H_{+}^{(10)}=0 & 3 H_{g-}^{(11)}-i H_{h+}^{(10)}=0 \\
i f H_{+}^{(32)}-\frac{3}{8} H_{g-}^{(33)}=0 & \frac{1}{f_{2}} H_{+}^{(31)}+\frac{1}{8} H_{h+}^{(32)}=0 \\
\frac{1}{f_{2}} H_{+}^{(03)}+f H_{+}^{(00)}=0 & 3 H_{g-}^{(01)}-i H_{h+}^{(00)}=0 \\
H_{h+}^{(33)}=0 & \frac{1}{f_{2}} H_{+}^{(30)}+f H_{+}^{(33)}-\frac{3 i}{8} H_{g-}^{(32)}=0 \\
3 i H_{g-}^{(21)}+H_{h+}^{(20)}=0 & \frac{1}{f_{2}} H_{+}^{(23)}+f H_{+}^{(20)}=0 \\
H_{+}^{(31)}=0 & \frac{1}{f_{2}} H_{+}^{(32)}-\frac{3 i}{8} H_{g-}^{(30)}-\frac{1}{8} H_{h+}^{(31)}=0
\end{array}
$$


Similar equations may be derived for the (9)-equations. In view of the first set of relations however, these will all be linearly dependent on the equations already obtained,and will thus not be needed.

\section{E.3 Third set of Hermitian relations}

A third set of Hermitian relations is obtained by eliminating $g_{z}$ and $g_{\bar{z}}^{*}$ terms between equations $(\mathrm{m})$, (i), and (9), contracting the resulting equations with symmetric $\tau^{(\alpha \beta)}$, taking differences to eliminate $D_{z} f$ term, and forming the linear combinations $(m)-(9)$,

$$
\begin{aligned}
& D_{z} \ln \left(f_{5} / f_{1}\right) \psi+\left(\frac{i}{f_{5}} \tau^{(22)}+\frac{\nu}{f_{1}} \tau^{(23)}\right) \xi^{*}-2 f \tau^{(12)} \xi^{*}+\frac{i}{2}\left(g_{9} \tau^{(13)} \psi^{*}-h \tau^{(12)} \psi^{*}\right)=0 \\
& D_{z} \ln \left(f_{5} / f_{1}\right) \xi-\left(\frac{i}{f_{5}} \tau^{(22)}+\frac{\nu}{f_{1}} \tau^{(23)}\right) \psi^{*}-2 f \tau^{(12)} \psi^{*}-\frac{i}{2}\left(g_{9}^{*} \tau^{(13)} \xi^{*}+h^{*} \tau^{(12)} \xi^{*}\right)=0
\end{aligned}
$$

Multiplying the above equations by $\xi^{t} \tau^{(\alpha \beta)}$ and $\psi^{t} \tau^{(\alpha \beta)}$ respectively, with $\tau^{(\alpha \beta) t}=\tau^{(\alpha \beta)}$, and taking the difference to eliminate the $D_{z} \ln \left(f_{5} / f_{1}\right)$ terms, we obtain,

$$
\begin{aligned}
& \xi^{t} \tau^{(\alpha \beta)}\left(\frac{i}{f_{5}} \tau^{(22)} \xi^{*}+\frac{\nu}{f_{1}} \tau^{(23)} \xi^{*}-2 f \tau^{(12)} \xi^{*}+\frac{i}{2} g_{9} \tau^{(13)} \psi^{*}-\frac{i}{2} h \tau^{(12)} \psi^{*}\right) \\
& +\psi^{t} \tau^{(\alpha \beta)}\left(\frac{i}{f_{5}} \tau^{(22)} \psi^{*}+\frac{\nu}{f_{1}} \tau^{(23)} \psi^{*}+2 f \tau^{(12)} \psi^{*}+\frac{i}{2} g_{9}^{*} \tau^{(13)} \xi^{*}+\frac{i}{2} h^{*} \tau^{(12)} \xi^{*}\right)=0
\end{aligned}
$$

Separating real and imaginary parts, we have

$$
\begin{array}{ll}
\frac{1}{f_{5}} H_{+}^{(22)}+\frac{1}{2} H_{g+}^{(13)}=0 & -\frac{\nu}{f_{1}} H_{+}^{(23)}-2 f H_{-}^{(12)}+\frac{i}{2} H_{h-}^{(12)}=0 \\
\frac{1}{f_{5}} H_{+}^{(23)}-\frac{1}{2} H_{g+}^{(12)}=0 & \frac{i \nu}{f_{1}} H_{+}^{(22)}-2 i f H_{-}^{(13)}-\frac{1}{2} H_{h-}^{(13)}=0 \\
\frac{1}{f_{5}} H_{+}^{(21)}+\frac{\nu}{f_{1}} H_{+}^{(20)}=0 & 2 i f H_{-}^{(11)}-\frac{i}{2} H_{g+}^{(10)}+\frac{1}{2} H_{h-}^{(11)}=0 \\
\frac{\nu}{f_{1}} H_{+}^{(33)}+\frac{1}{2} H_{g+}^{(03)}=0 & \frac{1}{f_{5}} H_{+}^{(32)}-2 f H_{-}^{(02)}+\frac{i}{2} H_{h-}^{(02)}=0 \\
\frac{\nu}{f_{1}} H_{+}^{(32)}+\frac{1}{2} H_{g+}^{(02)}=0 & \frac{i}{f_{5}} H_{+}^{(33)}-2 i f H_{-}^{(03)}-\frac{1}{2} H_{h-}^{(03)}=0 \\
\frac{i}{f_{5}} H_{+}^{(31)}+\frac{i \nu}{f_{1}} H_{+}^{(30)}-2 i f H_{-}^{(01)}+\frac{i}{2} H_{g+}^{(00)}-\frac{1}{2} H_{h-}^{(01)}=0 \\
\frac{i}{f_{5}} H_{+}^{(00)}+\frac{i \nu}{f_{1}} H_{+}^{(01)}-2 i f H_{-}^{(30)}+\frac{i}{2} H_{g+}^{(31)}-\frac{1}{2} H_{h-}^{(30)}=0 \\
\frac{1}{f_{5}} H_{+}^{(12)}-\frac{1}{2} H_{g+}^{(23)}=0 & -\frac{i \nu}{f_{1}} H_{+}^{(13)}+2 i f H_{-}^{(22)}+\frac{1}{2} H_{h-}^{(22)}=0 \\
\frac{1}{f_{5}} H_{+}^{(13)}+\frac{1}{2} H_{g+}^{(22)}=0 & \frac{\nu}{f_{1}} H_{+}^{(12)}+2 f H_{-}^{(23)}-\frac{i}{2} H_{h-}^{(23)}=0 \\
\frac{1}{f_{5}} H_{+}^{(11)}+\frac{\nu}{f_{1}} H_{+}^{(10)}=0 & -2 f H_{-}^{(21)}+\frac{1}{2} H_{g+}^{(20)}+\frac{i}{2} H_{h-}^{(21)}=0
\end{array}
$$




\section{Acknowledgments}

We wish to thank John Estes, Michael Gutperle, Per Kraus, Darya Krym, David Mateos, Diego Trancanelli for helpful discussions, and Andrew Royston and M.M. Sheikh Jabbari for useful correspondence.

Open Access. This article is distributed under the terms of the Creative Commons Attribution Noncommercial License which permits any noncommercial use, distribution, and reproduction in any medium, provided the original author(s) and source are credited.

\section{References}

[1] J.M. Maldacena, The large- $N$ limit of superconformal field theories and supergravity, Adv. Theor. Math. Phys. 2 (1998) 231 [Int. J. Theor. Phys. 38 (1999) 1113] [hep-th/9711200] [SPIRES].

[2] S.S. Gubser, I.R. Klebanov and A.M. Polyakov, Gauge theory correlators from non-critical string theory, Phys. Lett. B 428 (1998) 105 [hep-th/9802109] [SPIRES].

[3] E. Witten, Anti-de Sitter space and holography, Adv. Theor. Math. Phys. 2 (1998) 253 [hep-th/9802150] [SPIRES].

[4] O. Aharony, S.S. Gubser, J.M. Maldacena, H. Ooguri and Y. Oz, Large-N field theories, string theory and gravity, Phys. Rept. 323 (2000) 183 [hep-th/9905111] [SPIRES].

[5] E. D'Hoker and D.Z. Freedman, Supersymmetric gauge theories and the AdS/CFT correspondence, hep-th/0201253 [SPIRES].

[6] H. Lin, O. Lunin and J.M. Maldacena, Bubbling AdS space and 1/2 BPS geometries, JHEP 10 (2004) 025 [hep-th/0409174] [SPIRES].

[7] J.P. Gauntlett, D. Martelli, J. Sparks and D. Waldram, Supersymmetric AdS 5 solutions of type IIB supergravity, Class. Quant. Grav. 23 (2006) 4693 [hep-th/0510125] [SPIRES].

[8] J. Gomis and C. Romelsberger, Bubbling defect CFT's, JHEP 08 (2006) 050 [hep-th/0604155] [SPIRES].

[9] O. Lunin, 1/2-BPS states in M-theory and defects in the dual CFTs, JHEP 10 (2007) 014 [arXiv:0704.3442] [SPIRES].

[10] E. D'Hoker, J. Estes and M. Gutperle, Exact half-BPS Type IIB interface solutions I: Local solution and supersymmetric Janus, JHEP 06 (2007) 021 [arXiv:0705.0022] [SPIRES].

[11] E. D'Hoker, J. Estes and M. Gutperle, Exact half-BPS Type IIB interface solutions II: Flux solutions and multi-Janus, JHEP 06 (2007) 022 [arXiv:0705.0024] [SPIRES].

[12] J. Erdmenger, Z. Guralnik and I. Kirsch, Four-Dimensional Superconformal Theories with Interacting Boundaries or Defects, Phys. Rev. D 66 (2002) 025020 [hep-th/0203020] [SPIRES].

[13] E. D'Hoker, J. Estes, M. Gutperle and D. Krym, Exact Half-BPS Flux Solutions in M-theory I, Local Solutions, JHEP 08 (2008) 028 [arXiv:0806.0605] [SPIRES].

[14] E. D'Hoker, J. Estes, M. Gutperle and D. Krym, Exact Half-BPS Flux Solutions in M-theory II: Global solutions asymptotic to $A d S_{7} \times S^{4}, J H E P 12$ (2008) 044 [arXiv:0810.4647] [SPIRES]. 
[15] E. D'Hoker, J. Estes, M. Gutperle and D. Krym, Janus solutions in M-theory, JHEP 06 (2009) 018 [arXiv:0904.3313] [SPIRES].

[16] S. Yamaguchi, Bubbling geometries for half BPS Wilson lines, Int. J. Mod. Phys. A 22 (2007) 1353 [hep-th/0601089] [SPIRES].

[17] O. Lunin, On gravitational description of Wilson lines, JHEP 06 (2006) 026 [hep-th/0604133] [SPIRES].

[18] E. D'Hoker, J. Estes and M. Gutperle, Gravity duals of half-BPS Wilson loops, JHEP 06 (2007) 063 [arXiv:0705.1004] [SPIRES].

[19] E.I. Buchbinder, J. Gomis and F. Passerini, Holographic Gauge Theories in Background Fields and Surface Operators, JHEP 12 (2007) 101 [arXiv:0710.5170] [SPIRES].

[20] D. Gaiotto and E. Witten, Supersymmetric Boundary Conditions in $N=4$ Super Yang-Mills Theory, arXiv:0804.2902 [SPIRES].

[21] D. Gaiotto and E. Witten, Janus Configurations, Chern-Simons Couplings, And The Theta-Angle in $N=4$ Super Yang-Mills Theory, arXiv:0804.2907 [SPIRES].

[22] D. Gaiotto and E. Witten, S-duality of Boundary Conditions In $N=4$ Super Yang-Mills Theory, arXiv: 0807.3720 [SPIRES].

[23] E. D'Hoker, J. Estes, M. Gutperle, D. Krym and P. Sorba, Half-BPS supergravity solutions and superalgebras, JHEP 12 (2008) 047 [arXiv:0810.1484] [SPIRES].

[24] J.P. Gauntlett, D. Martelli, S. Pakis and D. Waldram, G-structures and wrapped NS5-branes, Commun. Math. Phys. 247 (2004) 421 [hep-th/0205050] [SPIRES].

[25] J.P. Gauntlett and S. Pakis, The geometry of D = 11 Killing spinors, JHEP 04 (2003) 039 [hep-th/0212008] [SPIRES].

[26] J.P. Gauntlett, D. Martelli, J. Sparks and D. Waldram, Supersymmetric AdS $S_{5}$ solutions of M-theory, Class. Quant. Grav. 21 (2004) 4335 [hep-th/0402153] [SPIRES].

[27] J.P. Gauntlett, Classifying supergravity solutions, Fortsch. Phys. 53 (2005) 468 [hep-th/0501229] [SPIRES].

[28] D. Gaiotto and J. Maldacena, The gravity duals of $N=2$ superconformal field theories, arXiv:0904. 4466 [SPIRES].

[29] L.F. Alday, D. Gaiotto, S. Gukov, Y. Tachikawa and H. Verlinde, Loop and surface operators in $N=2$ gauge theory and Liouville modular geometry, JHEP 01 (2010) 113 [arXiv:0909.0945] [SPIRES].

[30] D. Gaiotto, Surface Operators in $N=24 D$ Gauge Theories, arXiv:0911.1316 [SPIRES].

[31] I. Kirsch and D. Vaman, The D3/D7 background and flavor dependence of Regge trajectories, Phys. Rev. D 72 (2005) 026007 [hep-th/0505164] [SPIRES].

[32] J.A. Harvey and A.B. Royston, Gauge/Gravity duality with a chiral $N=(0,8)$ string defect, JHEP 08 (2008) 006 [arXiv:0804.2854] [SPIRES].

[33] O. Aharony, A. Fayyazuddin and J.M. Maldacena, The large- $N$ limit of $N=2,1$ field theories from three- branes in F-theory, JHEP 07 (1998) 013 [hep-th/9806159] [SPIRES].

[34] M. Graña and J. Polchinski, Gauge/gravity duals with holomorphic dilaton, Phys. Rev. D 65 (2002) 126005 [hep-th/0106014] [SPIRES].

[35] A. Karch and E. Katz, Adding flavor to AdS/CFT, JHEP 06 (2002) 043 [hep-th/0205236] [SPIRES]. 
[36] N.R. Constable, J. Erdmenger, Z. Guralnik and I. Kirsch, Intersecting D3-branes and holography, Phys. Rev. D 68 (2003) 106007 [hep-th/0211222] [SPIRES].

[37] M.M. Sheikh-Jabbari, unpublished (2008).

[38] E. D'Hoker, J. Estes, M. Gutperle and D. Krym, Exact Half-BPS Flux Solutions in M-theory III: Existence and rigidity of global solutions asymptotic to $A d S_{4} \times S^{7}$, JHEP 09 (2009) 067 [arXiv: 0906.0596] [SPIRES].

[39] E. D'Hoker, J. Estes and M. Gutperle, Ten-dimensional supersymmetric Janus solutions, Nucl. Phys. B 757 (2006) 79 [hep-th/0603012] [SPIRES].

[40] J.H. Schwarz, Covariant Field Equations of Chiral $N=2 D=10$ Supergravity, Nucl. Phys. B 226 (1983) 269 [SPIRES].

[41] P.S. Howe and P.C. West, The Complete $N=2, D=10$ Supergravity, Nucl. Phys. B 238 (1984) 181 [SPIRES]. 